\title{
Core Outcome Measures in Preclinical Assessment of Candidate Analgesics
}

\author{
S. Stevens Negus \\ Department of Pharmacology and Toxicology, Virginia Commonwealth University, Richmond, Virginia
}

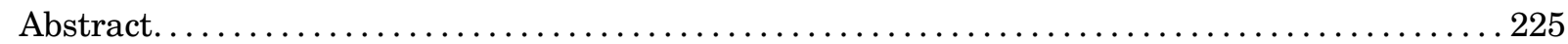

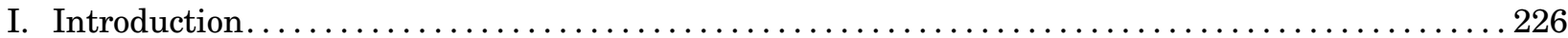

II. Behavioral Outcome Measures in Analgesic Drug Discovery $\ldots \ldots \ldots \ldots \ldots \ldots \ldots \ldots \ldots \ldots \ldots 228$

A. Unconditioned Behavior ............................................. 228

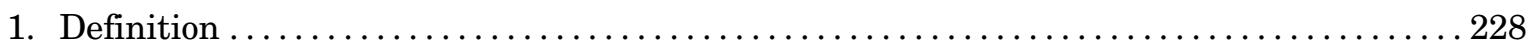

2. Pain Stimulus as an Unconditioned Stimulus . . . . . . . . . . . . . . . . . . . 229

3. Pain Stimulus as a Contextual Stimulus . . . . . . . . . . . . . . . . . . . . . . . . . 231

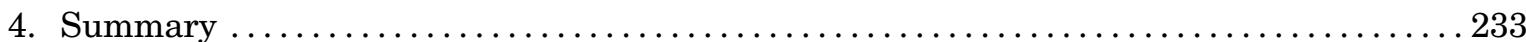

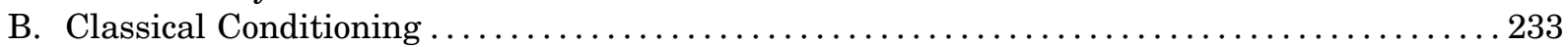

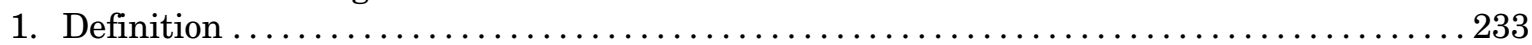

2. Pain Stimulus as the Unconditioned Stimulus $\ldots \ldots \ldots \ldots \ldots \ldots \ldots \ldots \ldots \ldots \ldots \ldots \ldots 234$

3. Pain Stimulus as a Contextual Stimulus ................................ 239

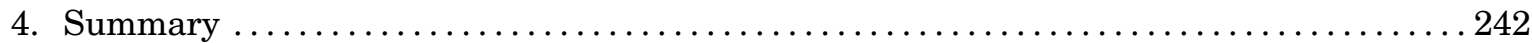

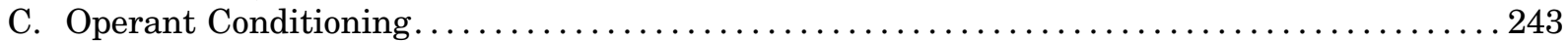

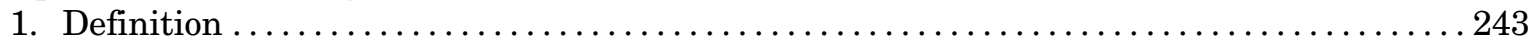

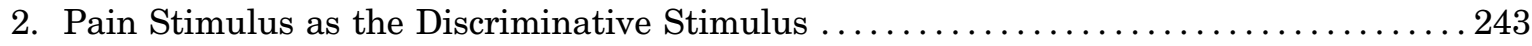

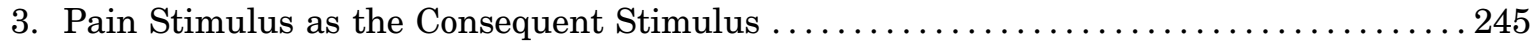

4. Pain Stimulus as a Contextual Stimulus .............................. 247

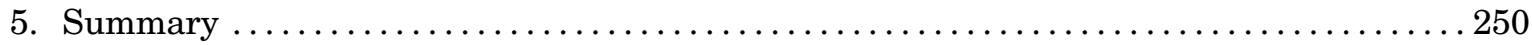

III. Preclinical Antinociceptive Drug Profiles ..................................... 250

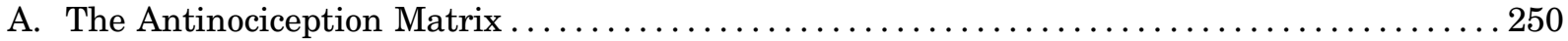

B. $\mu$ Opioid Receptor Agonists: A Representative Positive Control ...................... 252

C. $\kappa$ Opioid Receptor Agonists: A Representative Negative Control. .....................253

D. Cannabinoid Receptor Agonists: A Representative Class of Candidate Analgesics . . ...... 254

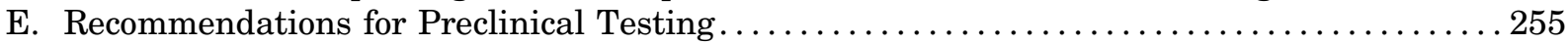

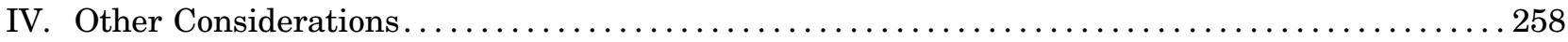

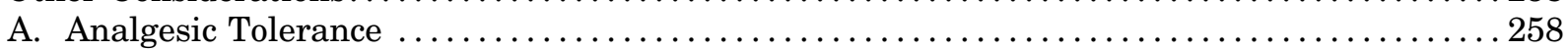

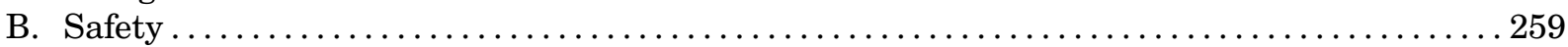

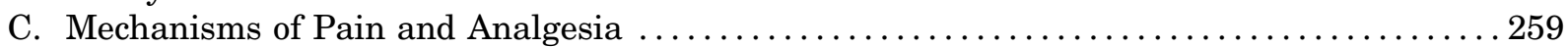

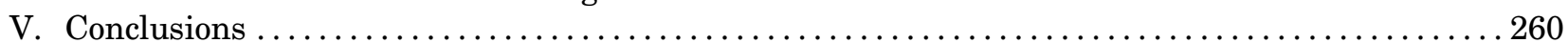

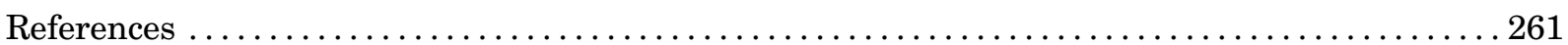

Abstract_-All preclinical procedures for analgesic drug discovery involve two components: 1) a "pain stimulus" (the principal independent variable), which is delivered to an experimental subject with the intention of producing a pain state; and 2) a "pain behavior" (the principal dependent variable), which is measured as evidence of that pain state. Candidate analgesics are then evaluated for their effectiveness to reduce the pain behavior, and results are used to prioritize drugs for advancement to clinical testing. This review describes a taxonomy of preclinical procedures organized into an "antinociception matrix" by reference to their types of pain stimulus (noxious, inflammatory, neuropathic, disease related) and pain

Address correspondence to: S. Stevens Negus, Department of Pharmacology and Toxicology, Virginia Commonwealth University, 410 N. 12th St., Richmond, VA 23298. E-mail: ssnegus@vcu.edu

This work was supported by the National Institutes of Health National Institute on Drug Abuse [Grant R01-DA030404] and the National Institutes of Health National Institute of Neurological Disorders and Stroke [Grant R01-NS070715].

https://doi.org/10.1124/pr.118.017210. 
behavior (unconditioned, classically conditioned, operant conditioned). Particular emphasis is devoted to pain behaviors and the behavioral principals that govern their expression, pharmacological modulation, and preclinical-to-clinical translation. Strengths and weaknesses are compared and contrasted for procedures using each type of behavioral outcome measure, and the following four recommendations are offered to promote strategic use of these procedures for preclinicalto-clinical analgesic drug testing. First, attend to the degree of homology between preclinical and clinical outcome measures, and use preclinical procedures with behavioral outcome measures homologous to clinically relevant outcomes in humans. Second, use combinations of preclinical procedures with complementary strengths and weaknesses to optimize both sensitivity and selectivity of preclinical testing. Third, take advantage of failed clinical translation to identify drugs that can be back-translated preclinically as active negative controls. Finally, increase precision of procedure labels by indicating both the pain stimulus and the pain behavior in naming preclinical procedures.

\section{Introduction}

Acute and chronic pain afflict millions of people each year at enormous cost in both health care and lost productivity (Institute of Medicine Committee on Advancing Pain Research, Care, and Education, 2011). The high prevalence of pain is a major cause of health care utilization (St. Sauver et al., 2013), and prescription and over-the-counter analgesics are among the most widely consumed drugs in the United States (Manchikanti et al., 2012; https://www.chpa.org/ SalesVolume.aspx). $\mu$ opioid receptor agonists in particular (e.g., morphine, hydrocodone, oxycodone, fentanyl, and methadone) are widely prescribed for treatment of relatively severe acute and chronic pain, although use of these drugs is limited by side effects that include abuse liability and potentially lethal respiratory depression (Pergolizzi et al., 2017). Overall, the prevalence of pain, demand for effective analgesics, and constraints on the use of existing drugs have driven a decades-long search for improved pain treatments, and the current crisis of opioid analgesic abuse and overdose deaths in the United States has invigorated this effort with new urgency (Volkow and Collins, 2017). Preclinical-to-clinical translational research from laboratory animals to humans has played a key role in analgesic drug development in the past and will likely continue to be important in the future as lessons from previous failures and successes are integrated into evolving research strategies (Negus et al., 2006; Yezierski and Hansson, 2018). This review will consider preclinical research strategies for candidate analgesic testing with a particular focus on behavioral outcome measures used to assess pain and the role of those outcome measures in the interpretation of drug effects.
Any preclinical procedure that aspires to pain measurement involves two components: 1) an experimental manipulation delivered to a research subject with the intent of producing a pain state (the principal independent variable, referred to below as the "pain stimulus"), and 2) the measurement of some change in behavior by that subject and interpreted as evidence of the pain state (the principal dependent variable, referred to below as the "pain behavior") (Negus et al., 2006; Vierck et al., 2008; Mogil, 2009; Clark, 2016; Whiteside et al., 2016). Once a model of "pain stimulus $\rightarrow$ pain behavior" has been established, then drugs can be evaluated for their effectiveness to reduce the pain behavior. For example, in a prototypical preclinical pain assay, delivery of a noxious heat stimulus to the tail of a mouse or rat can elicit a tail-withdrawal response. In this case, heat serves as the pain stimulus, the tail-withdrawal response serves as the pain behavior, and opioid analgesics such as morphine decrease that pain behavior. Parameters of the pain stimulus can be varied by altering its intensity, modality, or the anatomic site(s) to which it is applied, and clinical relevance can be further enhanced by incorporating treatments that produce inflammation, neuropathy, or other elements of pain-related injury or disease. Previous reviews have summarized advances in types of pain stimuli used to model clinically relevant pain states (Joshi and Honore, 2006; Mogil, 2009; Le Bars et al., 2010; Klinck et al., 2017; Munro et al., 2017), and those different approaches are summarized in Table 1.

Pain stimuli can produce a variety of changes in behavior, and a central challenge to analgesic drug discovery is the selection of outcome measures appropriate for study. Pain has been defined as "an unpleasant sensory and emotional experience associated with

\footnotetext{
ABBREVIATIONS: AMG9810, (E)-3-(4-t-butylphenyl)-N-(2,3-dihydrobenzo[b][1,4]dioxin-6-yl)acrylamide; CFA, complete Freund's adjuvant; CPA, conditioned place aversion; CPP, conditioned place preference; CP55940, 2-[(1R,2R,5R)-5-hydroxy-2-(3-hydroxypropyl) cyclohexyl]-5-(2-methyloctan-2-yl)phenol; CR, conditioned response in classical conditioning; CS, conditioned stimulus in classical conditioning; GR89696, methyl 4-[2-(3,4-dichlorophenyl)acetyl]-3-(pyrrolidin-1-ylmethyl)piperazine-1-carboxylate; ICSS, intracranial self-stimulation; MK-801, (5R,10S)-(+)-5-methyl-10,11-dihydro-5H-dibenzo[a,d]cyclohepten-5,10-imine; NMDA, $N$-methyl-Daspartate; NSAID, nonsteroidal anti-inflammatory drug; PAD, preclinical antinociception drug; PS, "pain stimulus" (i.e., a stimulus intended to produce a pain state); $\mathrm{R}$, response in operant conditioning; $\mathrm{S}^{\mathrm{C}}$, consequent stimulus in operant conditioning; $\mathrm{S}^{\mathrm{D}}$, discriminative stimulus in operant conditioning; THC, A9-tetrahydrocannabinol; UR, unconditioned response; US, unconditioned stimulus; U50,488, 2-(3,4-dichlorophenyl)-N-methyl-N-[(1R,2R)-2-pyrrolidin-1-ylcyclohexyl]acetamide; U69,593, $N$-methyl-2-phenyl- $N$ [(5R,7S,8S)-7-(pyrrolidin-1-yl)-1-oxaspiro[4.5]dec-8yl]acetamide; WIN55212-2, (11R)-2-methyl-11-[(morpholin-4-yl)methyl]-3-(naphthalene-1carbonyl)-9-oxa-1-azatricyclo[6.3.1.0 $\left.{ }^{4},{ }^{12}\right]$ dodeca-2,4(12),5,7-tetraene.
} 
TABLE 1

Taxonomy of pain stimuli and associated examples

\begin{tabular}{|c|c|c|}
\hline Pain Stimulus & Definition & Examples \\
\hline Acute noxious stimulus & Able to produce tissue damage & $\begin{array}{l}\text { Thermal-radiant heat } \\
\text { Mechanical-pressure } \\
\text { Chemical-acid }\end{array}$ \\
\hline Inflammatory \pm evocative acute stimulus ${ }^{a}$ & $\begin{array}{l}\text { Activates or mimics local or systemic inflammatory } \\
\text { processes }\end{array}$ & $\begin{array}{l}\text { CFA } \\
\text { Carrageenan } \\
\text { Surgical Incision }\end{array}$ \\
\hline Neuropathic \pm evocative acute stimulus ${ }^{a}$ & Damages peripheral sensory neurons or central neurons & $\begin{array}{l}\text { Nerve ligation or constriction } \\
\text { Chemotherapy }\end{array}$ \\
\hline Disease state \pm evocative acute stimulus ${ }^{a}$ & $\begin{array}{l}\text { Diabetes (e.g., streptozocin treatment) } \\
\text { Bone cancer (e.g., cancer cell injection to bone marrow) } \\
\text { Migraine (e.g., glycerol trinitrate injection) } \\
\text { Natural disease (e.g., arthritis in companion animals) }\end{array}$ & \\
\hline
\end{tabular}

${ }^{a}$ Models of inflammatory, neuropathic, or disease-state pain often involve two stimuli: one to produce a relatively sustained state of inflammation, neuropathy, or disease-

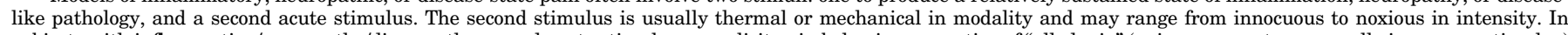

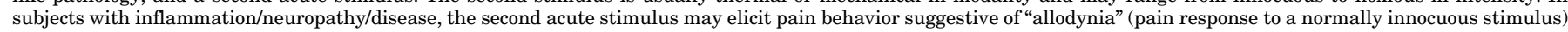
or "hyperalgesia" (hyperactive response to a normally noxious stimulus).

actual or potential tissue damage, or described in terms of such damage" (International Association for the Study of Pain, 1994). As such, pain is first and foremost a subjective experience that may be vivid and consuming to the subject in its grip; however, this subjective experience cannot be measured directly or objectively by an external observer. Instead, pain is expressed through the medium of behavior, and the detection of "pain," either in the clinic or the laboratory, is accomplished indirectly by assessment of behavior. Clinical pain assessment in verbally competent adults focuses largely on verbal behavior structured by instruments such as numeric rating scales or questionnaires (Dworkin et al., 2005; Melzack and Katz, 2013). In subjects that lack verbal competence (e.g., young children or laboratory animals), verbal behavior is not available as a source of dependent measures, and pain diagnosis relies instead on changes in other, nonverbal behaviors (Negus et al., 2006; von Baeyer et al., 2011; Berge, 2013; Li, 2013; McGrath and Unruh, 2013). This review will describe a taxonomy of nonverbal pain behaviors and discuss basic principles of behavioral analysis that govern their expression and pharmacological modulation in preclinical and translational research. The classes of behaviors to be described are summarized in Table 2.
The transduction of a pain stimulus into pain behavior is governed by both the evolutionary history (phylogeny) and the individual history (ontogeny) of the subject to which the stimulus is delivered. With regard to evolutionary history, the ability to detect and respond adaptively to noxious stimuli is fundamental to the survival of any organism, and natural selection operating over millennia has equipped members of any given species with genetic endowments that underlie anatomic traits and behavioral reflexes. Reflexive "unconditioned" pain behaviors have historically played a major role in preclinical pain research, and rodent tail-withdrawal responses from noxious heat exemplify this type of behavior. Reflexive behaviors are supplemented by customized stimulus-response relationships learned by individual organisms in the course of their own lifetimes via classical and operant conditioning. Conditioning enables individual organisms to learn new stimulus-response relationships tailored to the specific environments they inhabit, and conditioned behaviors are both prominent in pain expression by humans and increasingly important as outcome measures in preclinical pain research.

The remainder of this review will discuss basic principles of unconditioned, classically conditioned, and operant conditioned behaviors as they apply to

TABLE 2

Taxonomy of pain behaviors and associated examples

\begin{tabular}{|c|c|c|c|}
\hline Type of Behavior & Notation & Preclinical Examples & Clinical Correlates \\
\hline \multirow[t]{2}{*}{ Unconditioned behavior: US $\rightarrow$ UR } & $\mathrm{PS} \rightarrow \mathrm{UR}$ & $\begin{array}{l}\text { Stimulation of withdrawal responses, } \\
\text { writhing, flinching, grimacing }\end{array}$ & Same \\
\hline & $\mathrm{PS} \rightarrow[\mathrm{US} \rightarrow \mathrm{UR}]$ & $\begin{array}{l}\text { Depression of locomotion, feeding, wheel } \\
\text { running, burrowing, nesting }\end{array}$ & Impaired physical functioning \\
\hline \multirow[t]{2}{*}{$\begin{array}{l}\text { Classically conditioned behavior: } \\
\text { CS+US; CS } \rightarrow \text { CR }\end{array}$} & $\mathrm{PS}+\mathrm{CS} ; \mathrm{CS} \rightarrow \mathrm{CR}$ & $\begin{array}{l}\text { Conditioned place avoidance; fear } \\
\text { conditioning }\end{array}$ & Avoidance, anxiety, fear \\
\hline & $\mathrm{PS} \rightarrow[\mathrm{US}+\mathrm{CS} ; \mathrm{CS} \rightarrow \mathrm{CR}]$ & $\begin{array}{l}\text { Conditioned place preference to putative } \\
\text { analgesic treatments }\end{array}$ & Placebo analgesia \\
\hline \multirow[t]{2}{*}{ Operant behavior: $\mathrm{S}^{\mathrm{D}} \rightarrow \mathrm{R} \rightarrow \mathrm{S}^{\mathrm{C}}$} & $\mathrm{PS} \rightarrow \mathrm{R} \rightarrow \mathrm{S}^{\mathrm{C}}$ & Discrimination of a noxious stimulus & $\begin{array}{l}\text { Verbal reports of pain intensity or } \\
\text { quality }\end{array}$ \\
\hline & $\begin{array}{l}\mathrm{S}^{\mathrm{D}} \rightarrow \mathrm{R} \rightarrow \mathrm{PS} \\
\mathrm{PS} \rightarrow\left[\mathrm{S}^{\mathrm{D}} \rightarrow \mathrm{R} \rightarrow \mathrm{S}^{\mathrm{C}}\right]\end{array}$ & $\begin{array}{l}\text { Punishment with a noxious stimulus } \\
\text { Depression of operant responding for food } \\
\text { or brain stimulation }\end{array}$ & $\begin{array}{l}\text { Movement-induced pain } \\
\text { Impaired physical functioning }\end{array}$ \\
\hline
\end{tabular}


preclinical research on the expression of pain and the pharmacology of candidate analgesics. Specific examples will be provided, strengths and weaknesses of each approach will be discussed, and opportunities for new research will be presented. Additionally, basic principles of pain behavior apply to both laboratory animals and humans, and the implications of these principles for translational research will be considered. In particular, a major rationale for preclinical pain research is the prioritization of candidate analgesics for advancement to clinical testing. To serve this purpose, translational research evolves in an iterative cycle of forward and back translation to select for strategies that promote sensitivity to and selectivity for drugs that function as analgesics in humans (Fig. 1).

\section{Behavioral Outcome Measures in Analgesic Drug Discovery}

\section{A. Unconditioned Behavior}

1. Definition. An unconditioned response is any stimulus-induced physiologic or behavioral response that does not require learning for its expression (Gerrig and Zimbardo, 2002; Pierce and Cheney, 2017). Unconditioned responses are elicited by unconditioned stimuli, and the relationship can be described using the following terms:

$$
\mathrm{US} \rightarrow \mathrm{UR}
$$

where US denotes the unconditioned stimulus and UR denotes the unconditioned response. Unconditioned responses are often mediated by relatively simple neural circuits that include a sensory component (to detect the US) coupled to a motor component (to activate muscles associated with the UR), and genetic foundations for the development of these circuits have been selected during evolution to enable rapid expression of adaptive responses to stimuli that are both common in the environment where a species has evolved and physiologically relevant to individual members of that species. Most unconditioned responses do not involve pain stimuli. For example, food presentation in the mouth can serve as a US to elicit the UR of salivation, and increased ambient light can serve as a US to elicit the UR of pupillary contraction. However, a subset of unconditioned responses is elicited by "noxious stimuli," which can be defined as "stimuli that damage or threaten to damage normal tissues" (International Association for the Study of Pain, 1994). Noxious stimuli are typically thermal (e.g., heat), mechanical (e.g., pressure), or chemical (e.g., protons) in modality, and they are detected by specialized sensory receptors ("nociceptors") embedded in the damaged or threatened tissue (Sherrington, 1906; Woolf and Ma, 2007). Moreover, as with other US $\rightarrow$ UR relationships, unconditioned responses to noxious stimuli require some

\section{A Preclinical assay sensitivity and selectivity}

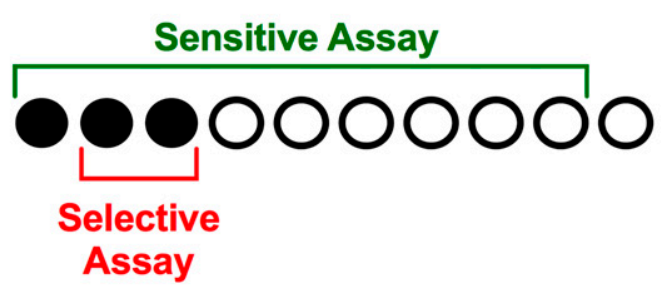

B Preclinical testing sequence

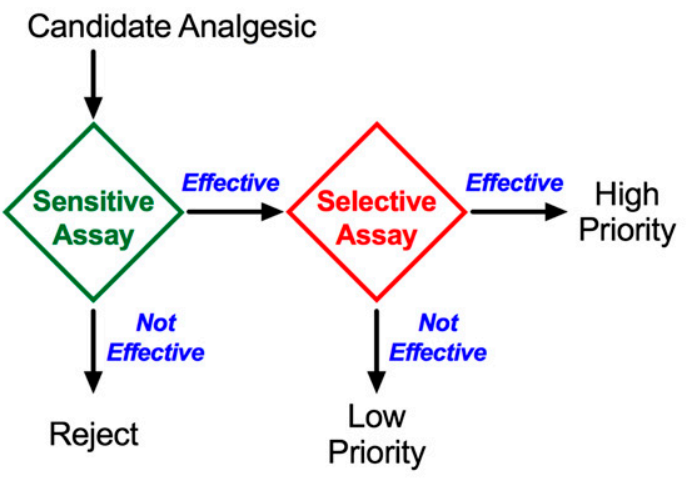

C Forward and back translation

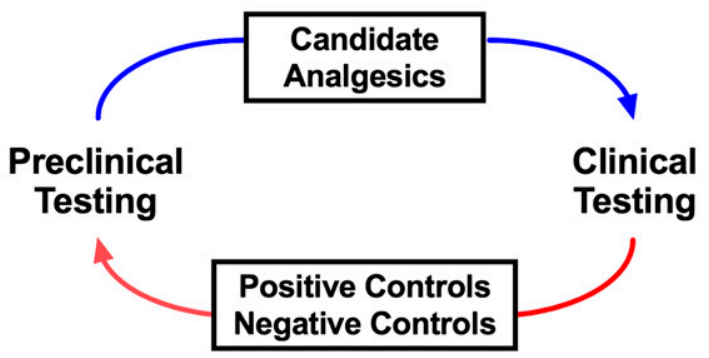

Fig. 1. Translational research involves an iterative process of forward and back translation to use and refine procedures that optimize sensitivity and selectivity for analgesic drugs. (A) A theoretical array of drugs that will ultimately function in humans as effective analgesics (filled circles) or as ineffective analgesics (open circles). The challenge in preclinical research is to identify the effective analgesics and filter out the ineffective compounds. Sensitive assays (green bracket) detect all or most true-positive analgesics but are vulnerable to false-positive effects with drugs that are not analgesics. Selective assays (red bracket) detect some or most true-positive analgesics and are less vulnerable to false positives with nonanalgesics; however, selective assays may yield false-negative results that fail to detect some analgesics. (B) A strategy to sequence assays with complementary characteristics of sensitivity and selectivity and prioritize candidate analgesics for human testing. An initial screen with a sensitive assay can identify a subset of drugs that includes both many true-positive analgesics but also some false positives. Additionally, these assays can be used to characterize attributes of in vivo pharmacology, including potency, efficacy, time course, and receptor mechanism(s) of action. Secondary testing with a selective assay can then screen out many false positives to identify high-priority drugs for advancement to human testing. Drugs effective in the sensitive assay but not in the selective assay may still have potential as analgesics (i.e., they may be false negatives in the selective assay), but such drugs would have lower priority for advancement. Drugs that are not effective in the sensitive assay can be rejected. (C) The iterative process of forward and back translation for use and refinement of preclinical procedures. In forward translation, candidate analgesics are prioritized for advancement to clinical studies. In back translation, drugs identified as effective or ineffective analgesics can be tested as positive or negative controls, respectively, and can also be used to refine preclinical procedures in ways that increase sensitivity to the positive controls and selectivity against negative controls. 
threshold level of stimulus intensity to elicit their expression, and increases in stimulus intensity above the threshold usually increase the rate, frequency, or intensity of the unconditioned response.

Unconditioned responses to noxious stimuli can be generally grouped into three different categories. First, noxious stimuli may elicit responses in physiologic subsystems within an organism without necessarily changing overt behavior. For example, a noxious heat stimulus may trigger action potentials in a subset of primary nociceptors, alter heart rate, or produce changes in patterns of cerebral blood flow (Apkarian et al., 2013; McGrath and Unruh, 2013; Ringkamp et al., 2013). Second, noxious stimuli may elicit withdrawal responses that have the effect of removing the affected tissue away from the stimulus (Berge, 2013). The heatinduced tail-withdrawal response described above illustrates this type of response, and it can be described as "behavior" insofar as it involves coordinated movement of skeletal musculature in a way that can be readily detected and measured by an observer. Lastly, noxious stimuli may elicit other types of motor behavior that can be reliably evoked, observed, and measured, although they may not result in withdrawal from the stimulus (Berge, 2013). Examples here include abdominal writhing/stretching behavior, facial grimacing, or vocalization produced by an inescapable stimulus such as intraplantar or intraperitoneal administration of a chemical irritant.

2. Pain Stimulus as an Unconditioned Stimulus. Measurement of the first category of unconditioned responses (i.e., physiologic responses) typically falls within the purview of physiologic research and will not be considered further here. The latter two categories, however, involve overt behaviors that can be readily detected by an external observer. For the purposes of this review, unconditioned behaviors elicited by noxious stimuli will be described using the following terms:

$$
\mathrm{PS} \rightarrow \mathrm{UR}
$$

where PS denotes the noxious "pain stimulus" and UR denotes the unconditioned response that serves as the primary outcome measure of pain-related behavior. These unconditioned behaviors have formed the foundation of preclinical research on pain and analgesic drug development for decades, and procedures to assess these behaviors have appeal for many reasons. Most importantly, these procedures possess a degree of face validity in relation to human behavior. For example, many of the unconditioned behaviors observed in laboratory animals (e.g., withdrawal from noxious heat) can also be observed in humans coincident with reports of pain (Lee and Stitzer, 1995; MontagneClavel and Oliveras, 1996), and some drugs (e.g., $\mu$ opioid receptor agonists) that decrease unconditioned pain-related behaviors in laboratory animals also decrease the sensation of pain in humans (Price et al., 1985).

In addition to advantages associated with face validity, these procedures are also associated with technical advantages that facilitate their use in research. For example, many pain-related unconditioned behaviors occur at low levels in the absence of noxious stimulation, they can be reliably elicited by presentation of a noxious stimulus to untrained or minimally trained experimental subjects, and they can be modified by drugs to permit pharmacological assessment of drug attributes such as potency, efficacy, time course, and receptor mechanism (Whiteside et al., 2016). A final noteworthy advantage is that stimulus-response relationships can be sensitized by manipulations that produce inflammation or neuropathy (Negus et al., 2006). Sensitized stimulus-response relationships provide evidence for "allodynia" (nociceptive response to a normally innocuous stimulus) or "hyperalgesia" (heightened nociceptive response to a normally noxious stimulus) (International Association for the Study of Pain, 1994), which are often interpreted as evidence for inflammatory or neuropathic pain. For example, both intraplantar injection of complete Freund's adjuvant (CFA; to produce paw inflammation) or injury to the sciatic nerve (to model neuropathy in sensory neurons that innervate the paw) result in hypersensitive paw withdrawal in response to mechanical stimulation (Bennett and Xie, 1988; Stein et al., 1988).

If these procedures were sufficient for accurate translational research on the expression, mechanisms, and treatment of pain, then this review would not be necessary. They are not sufficient, and recent failures in analgesic drug development have exposed key weaknesses (Pande et al., 1996; Hill, 2000; Huggins et al., 2012; Fallon et al., 2017; Yezierski and Hansson, 2018). In considering these weaknesses, it is perhaps best to begin with a point of logic. Although a noxious stimulus may elicit both an unconditioned response and the sensation of pain in humans, it does not follow that the unconditioned response $=$ pain, or that reducing the unconditioned response $=$ a reduction in pain. This type of distinction between unconditioned responses and perceptual experiences is obvious in other stimulus modalities. In vision, for example, a dark theater may be suddenly illuminated by the opening scene of Star Wars (https://www.youtube.com/watch?v=yHfLyMAHrQE). Light from the screen will cause both an unconditioned response of pupillary constriction and a perceptual response of the words "Star Wars" outlined in yellow and receding against a star-filled night sky. These different responses, although caused by a common stimulus, differ in their underlying mechanisms and in their responses to drugs. For example, topical application of a muscarinic acetylcholine receptor antagonist like tropicamide might block pupillary constriction without affecting accuracy of perception, 
whereas a serotonin $2 \mathrm{~A}$ receptor agonist hallucinogen like psilocybin might alter perception while having little effect on pupillary constriction.

In support of this distinction for nociception, a footnote to the definition of "pain" promulgated by the International Association for the Study of Pain (1994) points out that unconditioned "activity induced in the nociceptor and nociceptive pathways by a noxious stimulus is not pain, which is always a psychological state." It could be added that unconditioned behavioral responses to a noxious stimulus are also "not pain," and this distinction implies that mechanisms and modulators of pain in humans may differ from mechanisms and modulators of the unconditioned physiologic and behavioral responses to noxious stimuli commonly used as outcome measures in preclinical pain research. One of the most prominent examples of this distinction lies in the vulnerability of conventional preclinical procedures to treatments that produce motor impairment and behavioral depression. Specifically, common painrelated unconditioned behaviors (e.g., withdrawal reflexes) are "pain-stimulated behaviors," which can be defined as behaviors that increase in rate, frequency, or intensity after delivery of a noxious stimulus. These behaviors can be reduced by treatments that decrease sensory sensitivity to the noxious stimulus (true analgesia); however, these behaviors can also be reduced by treatments that impair the subject's motoric ability to emit the unconditioned response (e.g., by causing sedation or paralysis, or by increasing a competing behavior). Treatments that produce nonselective motor impairment often produce falsepositive analgesic-like effects in conventional assays of pain-stimulated behavior.

Figure 2 illustrates the potential for misleading results from a preclinical study of drug effects on unconditioned pain-stimulated behavior (Bagdas et al., 2016).
In this procedure, intraperitoneal administration of dilute acid (referred to here as "intraperitoneal acid") to mice served as an acute visceral noxious stimulus (the PS) to evoke an unconditioned response ("stretching" or "writhing," the UR). This UR was reduced by the clinically effective $\mu$ opioid analgesic drug morphine; however, stretching was also dose-dependently reduced by the centrally acting and pharmacologically selective $\kappa$ opioid receptor agonist 2-(3,4-dichlorophenyl)N-methyl-N-[(1R,2R)-2-pyrrolidin-1-ylcyclohexyl]acetamide (U50,488). U50,488 has not been tested in humans, but other centrally acting $\kappa$ agonists performed poorly in clinical trials, and $\kappa$ agonists are not approved for pain treatment anywhere in the world (Pande et al., 1996; Lazenka et al., 2018).

Accordingly, the apparent antinociceptive effect of U50,488 in the assay of intraperitoneal acid-stimulated stretching represents a false-positive effect with regard to preclinical-to-clinical forward translation, and this type of false positive has motivated efforts to refine preclinical testing procedures and improve predictive validity. The most common strategy to address this issue has been to compare drug potency in assays of unconditioned pain-stimulated behavior with drug potency in assays of motor performance, such as performance on a rotarod apparatus. The reasoning is that a given drug is more likely to function as an analgesic in humans if it produces preclinical antinociception at doses that do not produce signs of motor impairment in other behavioral procedures. However, relative potency to produce antinociception versus motor impairment has not proven to be a reliable predictor of analgesic potential. For example, one study found that both $\mu$ and $\kappa$ opioid agonists had similar potency ratios for antinociception versus motor impairment on a rotarod task (Seguin et al., 1995), but only $\mu$ agonists are approved as analgesics.

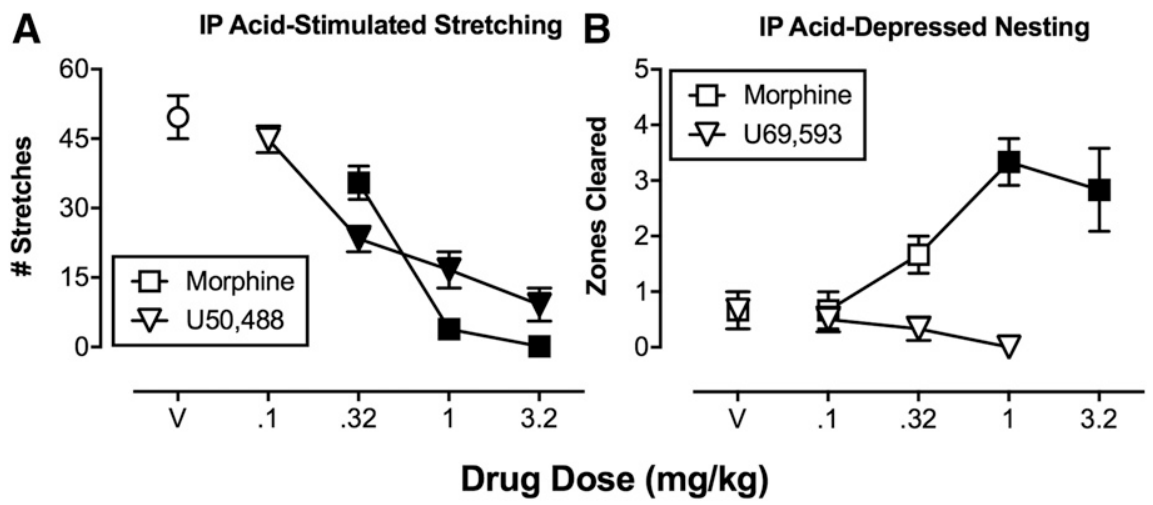

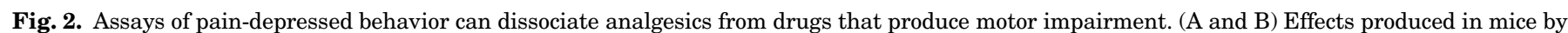

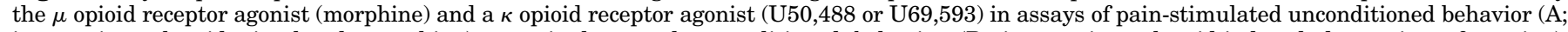

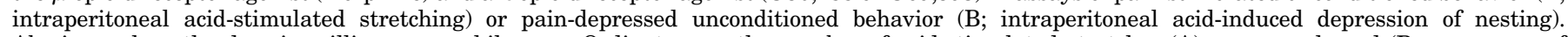

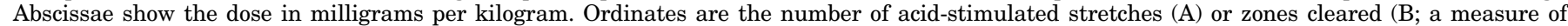

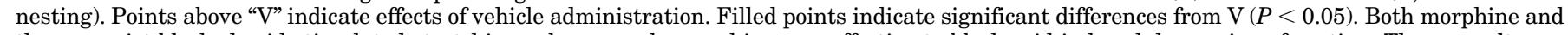

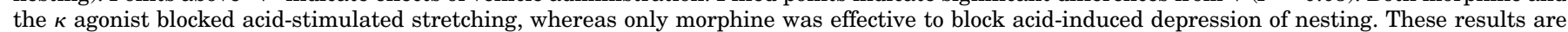

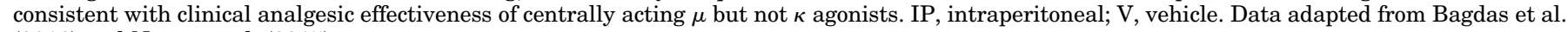
(2016) and Negus et al. (2015). 
Two other points warrant mention with regard to the translational utility of conventional preclinical assays of pain-stimulated behavior. First, although both humans and nonhumans exhibit withdrawal reflexes from noxious stimuli, analgesic drugs are rarely used to block these withdrawal reflexes in humans. For example, medical procedures (e.g., surgery) are perhaps the most common context within which humans are treated with drugs to reduce pain-stimulated behaviors evoked by noxious stimuli (e.g., skin incision with a scalpel blade); however, drugs used in this context are usually local or general anesthetics (i.e., drugs that block all sensory modalities in the affected tissue) rather than analgesics (drugs that selectively reduce nociception without affecting other sensory modalities) (Whitlock and Pardo, 2017). Second, the use of pain-stimulated unconditioned behaviors to assess allodynia and hyperalgesia in the context of inflammation and neuropathy is also problematic. Similar evocative stimuli are often used to assess allodynia and/or hyperalgesia in both laboratory animals and in humans (e.g., von Frey filaments to assess mechanical sensitivity; Chaplan et al., 1994; Kostek et al., 2016); however, the behavioral outcome measures in laboratory animals and humans are dramatically different. Specifically, the terms "allodynia" and "hyperalgesia" refer to hypersensitivity of painstimulated unconditioned behaviors (e.g., paw withdrawal) in laboratory animals, but to hypersensitivity of verbal pain endorsement in humans, often in the absence of an accompanying withdrawal reflex. Thus, just as painstimulated unconditioned behaviors are not equivalent to the human experience or report of pain, preclinical measures of allodynia and hyperalgesia are not equivalent to clinical measures of allodynia and hyperalgesia in humans.

In summary, measures of pain-stimulated unconditioned behavior in laboratory animals are not equivalent to core outcome measures of pain in humans, they do not serve as reliable surrogate measures of pain, and treatment-induced decreases in pain-stimulated unconditioned behavior do not provide reliable evidence of analgesia. With regard to assay characteristics of sensitivity and selectivity (Fig. 1), assays of painstimulated behavior are sensitive but not selective.

3. Pain Stimulus as a Contextual Stimulus. In addition to stimulating unconditioned behaviors, noxious stimuli can also be part of the context in which other US $\rightarrow$ UR relationships are expressed. This relationship can be expressed as follows:

$$
\mathrm{PS} \rightarrow[\mathrm{US} \rightarrow \mathrm{UR}]
$$

where PS again denotes the noxious pain stimulus, US indicates a non-noxious unconditioned stimulus, and UR indicates the unconditioned response elicited by the US. In cases in which the pain stimulus is a contextual stimulus, expression of the unconditioned response is often depressed, and "pain-depressed behaviors" can be defined as behaviors that decrease in rate, frequency, or intensity after presentation of a noxious stimulus (Negus et al., 2010a; Negus, 2018). For example, presentation of nesting material (the US) elicits nesting behavior (the UR) in many strains of mice under normal laboratory conditions, and noxious stimuli such as intraperitoneal acid can then serve as a contextual pain stimulus to depress nesting (Gaskill et al., 2013; Jirkof, 2014; Negus et al., 2015; Lewter et al., 2017; Oliver et al., 2018). Many other examples of pain-depressed unconditioned behavior have been reported, including depression by noxious stimuli of feeding (elicited by presentation of palatable food) (Stevenson et al., 2006; Kwilasz and Negus, 2012), locomotor activity (elicited by a novel environment) (Matson et al., 2007; Stevenson et al., 2009), burrowing (elicited by presentation of a cylinder filled with a substrate such as gravel) (Andrews et al., 2012; Rutten et al., 2014a; Wodarski et al., 2016; Shepherd et al., 2018), and wheel running (elicited by access to a running wheel) (Miller et al., 2011; Stevenson et al., 2011; Cobos et al., 2012; Kandasamy et al., 2016).

There are at least two potential benefits to incorporating assays of pain-depressed behavior into preclinical research on pain and analgesic drug development. The first is face validity. Behavioral depression (also known by other names such as "functional impairment" or "reduced activities of daily living") is a core outcome measure of pain in human and veterinary medicine, and alleviation of pain-depressed behavior is often a primary goal of treatment. For example, a set of core outcome measures has been recommended for use in human clinical trials of candidate analgesics by the Initiative on Methods, Measurement, and Pain Assessment in Clinical Trials (Dworkin et al., 2005). The primary type of pain measure recommended by this group was verbal report of pain intensity and quality (to be discussed in section II.C.2), but the secondary recommendation was for measures of pain-depressed behavior assessed using instruments such as the Brief Pain Inventory or McGill Pain Questionnaire. The Parents' Postoperative Pain Measure is an especially instructive instrument for comparison with preclinical research approaches because it is intended for use with young children who may lack verbal competency, and it relies not on self-reports by the subject but on assessment of overt behavior by an external observer (in this case, the parents) (Chambers et al., 1996, 2003; von Baeyer et al., 2011). The short form of the Parents' Postoperative Pain Measure evaluates 10 endpoints, five of which reflect behavioral depression (e.g., "Play less than usual"), whereas the other five reflect painstimulated behaviors (e.g., "Whine or complain more than usual"). Notably, in a study of the instrument's validity in postsurgical patients, parents endorsed more signs of pain-depressed than pain-stimulated behavior in their children (von Baeyer et al., 2011). Assessment of 
pain-depressed behaviors also plays a major role in veterinary pain diagnosis. For example, a canine Brief Pain Inventory asks dog owners to rate pain-depressed behaviors in their pets (Brown et al., 2008). As another example, federal guidance on pain assessment in laboratory animals focuses on measures of depressed feeding and associated weight loss, depressed locomotor activity, and depressed grooming (National Research Council, 2011). The focus on pain-depressed behaviors in clinical pain diagnosis, especially when verbal reports are unavailable, suggests that measures of paindepressed behaviors might also be a valuable source of endpoints in preclinical research.

A second advantage of pain-depressed behavior as an experimental endpoint is that drugs producing motor impairment do not produce false-positive analgesic-like effects. This results in increased assay selectivity. Specifically, in assays of pain-depressed behavior, the noxious stimulus itself decreases the behavioral endpoint, and analgesic drugs attenuate this behavioral depression to increase the target behavior. By contrast, drugs that produce motor impairment only exacerbate pain-related behavioral depression. For example, Fig. 2 shows effects of morphine and the centrally acting $\kappa$ agonist $N$-methyl-2-phenyl- $N$-[(5R,7S,8S)-7-(pyrrolidin1-yl)-1-oxaspiro[4.5]dec-8yl] acetamide (U69,593) on intraperitoneal acid-induced depression of nesting in mice (Negus et al., 2015). As noted above, intraperitoneal acid administration can serve as an acute visceral noxious stimulus that depresses nesting behavior. Morphine dose-dependently blocked intraperitoneal acid-induced depression of nesting, consistent with its known effectiveness as an analgesic; however, in this assay of paindepressed behavior, the $\kappa$ agonist failed to produce a morphine-like effect. The failure of U69,593 to alleviate acid-induced depression of nesting is consistent with the lack of clinical evidence for analgesic effectiveness and safety of centrally acting $\kappa$ agonists (Pande et al., 1996; Lazenka et al., 2018).

In addition to these strengths, assays of paindepressed behavior also present challenges. Most important among these is identification of conditions that generate high, stable rates of baseline behavior and reliable depression of that behavior by a noxious stimulus of interest. For example, a consortium of European investigators at eight different industry and academic research sites recently evaluated the reliability with which intraplantar CFA reduced burrowing behavior by rats (Wodarski et al., 2016). The study concluded that both baseline burrowing behavior and intraplantar CFA-induced depression of burrowing were generally reproducible across sites; however, the study also revealed within- and between-site variability in both baseline and intraplantar CFAdepressed behavior and began to identify factors (e.g., rat weight, time after intraplantar CFA administration, size of the burrowing substrate) that might influence behavioral outcomes. Other studies have found that putative inflammatory-, neuropathic-, and disease-related pain manipulations produce either little evidence for behavioral depression or a shorter duration of pain-depressed than pain-stimulated behaviors (Urban et al., 2011; Cobos et al., 2012; Grace et al., 2014; Forte et al., 2016; Okun et al., 2016; Sheahan et al., 2017). For example, treatment of mice with the chemotherapy drug paclitaxel was sufficient to produce hypersensitive paw withdrawal in response to mechanical stimuli, a pain-stimulated behavior commonly interpreted as evidence of neuropathic pain; however, this paclitaxel treatment regimen was not sufficient to depress nesting behavior (Toma et al., 2017). Taken together, these results suggest that many preclinical models of inflammatory-, neuropathic-, and disease-related pain fail to produce a full spectrum of pain-related behaviors in laboratory animals and may not be adequate for research on treatments for dimensions of pain that involve behavioral depression and functional impairment.

A second challenge in assays of pain-depressed unconditioned behavior is that these baseline behaviors can be depressed by innocuous as well as noxious stimuli. As a result, demonstration that a given stimulus depresses behavior cannot be interpreted as evidence of pain, and interpretation of depressed behavior as "pain-related" requires other supporting evidence. For example, one study investigated the effects of noxious and innocuous stimuli on consumption of a palatable food in rats. In this study, rats reliably consumed a large number of food pellets under baseline conditions, and this consumption could be reduced either by a noxious stimulus (intraperitoneal acid) or by an innocuous stimulus (prefeeding to induce satiety) (Kwilasz and Negus, 2012). Notably, the nonsteroidal anti-inflammatory drug (NSAID) ketoprofen blocked depression of feeding by intraperitoneal acid but not by prefeeding. The sensitivity to a clinically effective analgesic supported the conclusion that intraperitoneal acid-induced depression of feeding was pain related, whereas prefeeding-induced depression of feeding was not.

As a third issue, assays of pain-related behavioral depression are theoretically vulnerable to treatments that produce nonselective behavioral stimulation. Thus, just as pain-stimulated behaviors may be reduced by treatments that produce false-positive motor impairment, pain-depressed behaviors might be restored by treatments that produce false-positive motor activation. This issue can be addressed experimentally by comparing effects of a given treatment on behavior depressed by a noxious stimulus with effects of that same treatment on behavior depressed by a non-noxious stimulus. For example, as noted above, the NSAID ketoprofen blocked intraperitoneal acid-induced depression of feeding in rats, but it did not block prefeeding-induced 
depression of feeding, and it also did not alter baseline feeding in the absence of a depressant stimulus (Kwilasz and Negus, 2012). In this case, the selectivity of ketoprofen to block depression of feeding by a putative noxious stimulus supported the conclusion that ketoprofen effects resulted from true analgesia and not from general motor activation. This issue can also be addressed experimentally by comparing effects of test drugs with effects of stimulants like amphetamine. For example, intraplantar CFA administration reduced both horizontal and vertical (rearing) locomotor activity in rats (Matson et al., 2007). The NSAID ibuprofen alleviated both effects, whereas amphetamine alleviated CFA-induced depression of horizontal but not vertical locomotion. The authors concluded that vertical locomotion was less vulnerable than horizontal locomotion to general stimulant effects, and accordingly, they focused on vertical locomotion as the primary endpoint in evaluating other drugs.

A related theoretical point also warrants mention regarding the relative vulnerability of pain-related behaviors to nonselective motor impairment or activation. On the one hand, it is possible for a treatment to produce nonselective depression of all behavior; an extreme example is the elimination of all behavior by paralysis or death. On the other hand, it is less possible for a treatment to produce nonselective behavioral stimulation. An organism can engage in only a subset of behaviors at a time, and although periods of inactivity might be followed by expression of new behaviors, at some point, activation of any one behavior necessarily competes with and reduces expression of other behaviors. Thus, a treatment that increases some behaviors may not increase other behaviors (e.g., stimulation of a horizontal but not vertical locomotor activity by amphetamine in the example above) and may in fact decrease expression of other behaviors. For this reason, false-positive effects are more likely to be produced by nonselective motor impairment in assays of painstimulated behavior than by nonselective motor stimulation in assays of pain-depressed behavior.

A final weakness of assays of pain-depressed behavior is that they may fail to detect analgesic effects of drugs that also produce general disruption of behavior. As one example, it was noted above that intraplantar CFA reduced rearing in rats (Matson et al., 2007). This CFA-induced depression of vertical activity could be blocked by an intermediate morphine dose $(1.0 \mathrm{mg} / \mathrm{kg})$ but not by a higher dose $(3.0 \mathrm{mg} / \mathrm{kg})$ that robustly decreased rearing in the absence of the acid noxious stimulus. These results were interpreted to suggest that sedative effects obscured detection of antinociception at high morphine doses. More generally, morphine and other $\mu$ agonists usually produce antinociception at subsedative dose in assays of paindepressed behavior (although not always; Gould et al., 2016). However, the sensitivity of these assays will be low for true analgesics that produce motor disruption at doses equal to or less than doses that produce analgesia.

4. Summary. Noxious stimuli produce both painstimulated unconditioned behaviors and depression of other unconditioned behaviors, and both types of changes in behavior can serve as outcome measures in preclinical research on the mechanisms and treatment of pain. For example, intraperitoneal acid both stimulates a stretching/writhing response and depresses other unconditioned behaviors such as feeding, locomotion, and nesting in mice. Complementary use of assays that measure both classes of behavior can improve translational pain research, because clinical pain states involve both classes of behavior, and treatments that block sensitivity to the noxious stimulus should block both types of behavior. Moreover, coordinated use of these approaches can improve preclinical-to-clinical translation of drug effects as described in Fig. 1, because assays of pain-stimulated and pain-depressed behavior have relatively high sensitivity and selectivity, respectively.

\section{B. Classical Conditioning}

1. Definition. Classical conditioning is a type of learning in which one stimulus (the conditioned stimulus, CS) gains effectiveness to produce a behavioral response (the conditioned response, CR) after being paired with an unconditioned stimulus (Fanselow and Wassum, 2015; Pierce and Cheney, 2017). This learned relationship between stimuli leading to new responses can be diagrammed as follows:

$$
\text { 1) } \mathrm{CS}+\mathrm{US} \text {; 2) } \mathrm{CS} \rightarrow \mathrm{CR}
$$

The CS is typically a stimulus that initially elicits little behavioral response, whereas the US elicits an unconditioned response as described above in section II.A (US $\rightarrow$ UR). Classical conditioning proceeds in two phases. First, during conditioning, the CS and US are presented together in a regimen of paired stimulus presentations (CS+US). Second, during testing, the CS is presented alone, and behavioral effects of the CS are evaluated. If conditioning has occurred, then the CS will elicit a $\mathrm{CR}(\mathrm{CS} \rightarrow \mathrm{CR})$. The prototypical example of classical conditioning is the "Pavlov's Dog" experiment, in which the CS was a ringing bell, the US was food presentation, and the UR was salivation; after repeated pairing of the ringing bell with food, the sound of the bell alone came to elicit the CR of salivation (Pavlov, 1927). In general, conditioned responses have the adaptive function of preparing the subject for the US, and CRs may be similar to or different from the UR. In the case of the "Pavlov's Dog" experiment, the ringing bell CS elicited a salivation CR in anticipation of food delivery.

The determinants of classical conditioning have been a topic of intense study for decades, and numerous basic 
principles have been identified (Fanselow and Wassum, 2015; Pierce and Cheney, 2017). Two will be mentioned here. First, the effectiveness of a regimen of CS+US conditioning is influenced in part by the temporal relationship of CS and US presentation (Fig. 3). In general, conditioning is most effective when the CS begins before and continues during presentation of the US (delay conditioning). Conditioning is less effective or ineffective when the CS is terminated before onset of the US (trace conditioning), delivered with an onset and offset identical to the US (simultaneous conditioning), or delivered after US onset (backward conditioning). Indeed, in backward conditioning, the CS may become associated with termination of the US rather than with its delivery. Second, conditioning is most effective when both the CS and US are reliably presented together. For example, conditioning is strongest when both the CS and US are novel at the outset of conditioning and are always presented together, whereas conditioning is weaker if the subject has had prior exposure either to the CS without the US (latent inhibition) or to the US without the CS (US pre-exposure effect). Moreover, an established $\mathrm{CS} \rightarrow \mathrm{CR}$ relationship can be weakened by repeated subsequent presentations of the CS without the US (extinction).

In a second basic principle of classical conditioning, different experimental designs can be used to examine effects of drugs or other manipulations on either the development or the expression of a conditioned response. In studies to evaluate treatment effects on the development of a CR, the treatment is administered during the conditioning phase of the study (for example, as a pretreatment to each iteration of CS+US pairings). Under this type of design, treatments can modify the ultimate expression of the CR on the test day by modifying either 1) sensitivity to the CS or the US or 2) memory consolidation processes responsible for the association of the CS and US. By contrast, in studies to evaluate treatment effects on the expression of the CR, the treatment is administered only during the test phase, after the conditioning phase has been completed. Treatments can modify CR expression by modifying 1 ) sensitivity to the CS, 2) memory recall processes that link the CS to the CR, or 3) motor competence to perform the CR.

2. Pain Stimulus as the Unconditioned Stimulus. In one type of classical conditioning design used in preclinical pain research, noxious "pain" stimuli play the role of the US. This role can be expressed as follows, with PS again denoting the pain stimulus:

$$
\text { 1) } \mathrm{CS}+\mathrm{PS} ; 2) \mathrm{CS} \rightarrow \mathrm{CR}
$$

In this type of experiment, the PS is presented only during development of the $\mathrm{CR}$ in the conditioning phase, and as a result, candidate analgesic drugs are

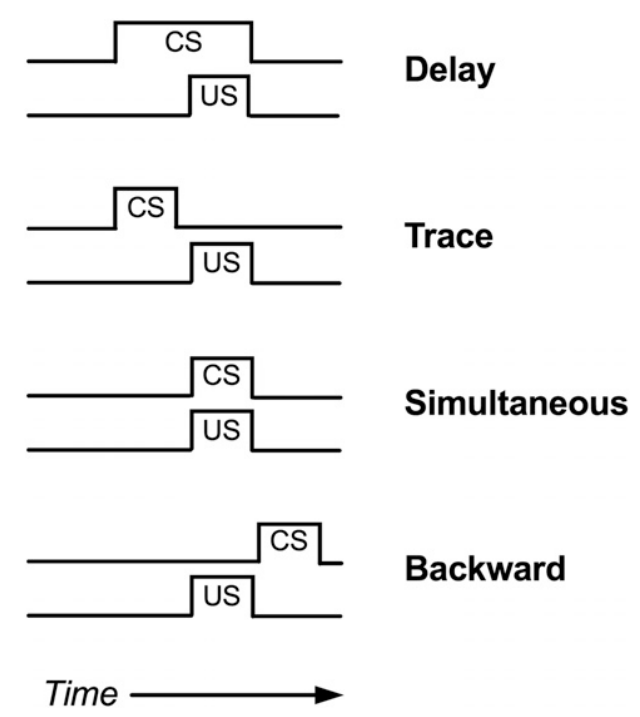

Fig. 3. Possible temporal alignments of a conditioned stimulus (CS) and unconditioned stimulus (US) in classical conditioning. Classical conditioning is most effective with "delay conditioning."

also administered during the conditioning phase to assess drug effectiveness to block development of the CR. One example of this approach is "conditioned place aversion" (CPA) (Johansen et al., 2001; Tanimoto et al., 2003; Deyama et al., 2007; van der Kam et al., 2008; Wang et al., 2009; Bagdas et al., 2016), and Fig. 4 shows data from an example of a CPA study using a noxious stimulus as the US (Bagdas et al., 2016). Placeconditioning studies typically use an apparatus with at least two compartments (i.e., two "places") distinguished by different visual, tactile, and/or olfactory stimuli and separated by a removable barrier. For the study shown in Fig. 4, the compartments had different wall colors and floor textures (black walls with a floor of parallel bars vs. white walls with a mesh floor). During conditioning, the barrier between compartments was in place, and one group of mice was treated with a noxious stimulus (intraperitoneal acid at a concentration sufficient to elicit stretching as a UR) prior to confinement for 40 minutes in one compartment, and with intraperitoneal saline prior to confinement for 40 minutes in the other compartment. Both types of conditioning session were conducted on the same day at least 4 hours apart, and the particular compartment paired with intraperitoneal acid was counterbalanced across mice. Thus, the distinguishing visual and tactile stimuli of the acidpaired compartment served as the CS, and the intraperitoneal acid injection served as the PS. A control group of mice was treated with vehicle in both compartments. Testing occurred 1 day later by placing mice back into the apparatus with the barrier removed so that mice could move freely between the two compartments for 15 minutes. Mice conditioned with intraperitoneal acid spent less time in the acid-paired compartment after conditioning than during a preconditioning trial, and this decrease in time spent in the 


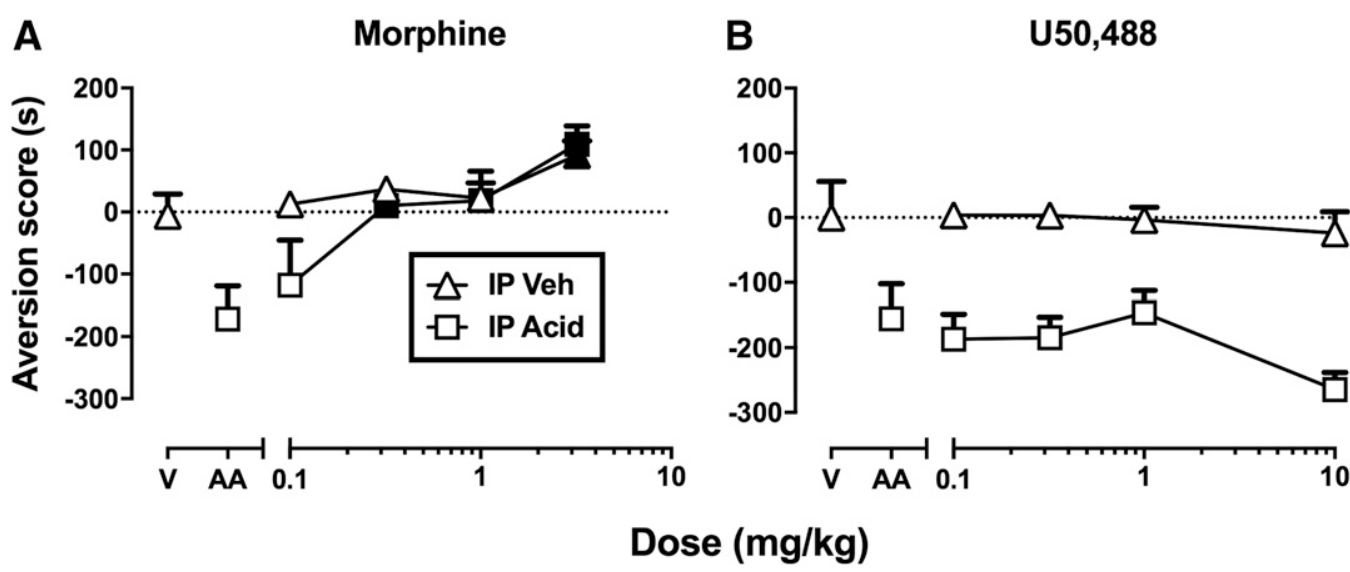

Fig. 4. Assays of pain-related CPA can dissociate analgesics from nonanalgesics. (A and B) Effects produced in mice by the $\mu$ opioid receptor agonist analgesic morphine (A) or the $\kappa$ opioid receptor agonist nonanalgesic U50,488 (B) in mice also conditioned with intraperitoneal (IP) acetic acid (AA) or in vehicle-treated controls (V). Abscissae show the dose in milligrams per kilogram. Ordinates are the aversion score expressed as time in seconds in the acid- or vehicle control-paired compartment after conditioning minus time in that compartment before conditioning. AA administered alone produced a CPA, whereas V did not. Morphine blocked acid-induced CPA in acid-treated mice more potently than it produced a conditioned place preference in vehicle-treated mice. Filled points in $(\mathrm{A})$ indicate a significant difference from $\mathrm{V}$ or AA after morphine treatment $(P<0.05)$. Conversely, U50,488 failed to alter either acid-induced CPA in acid-treated mice or preference in the vehicle-treated mice. U50,488 was tested up to doses that blocked acid-stimulated stretching (see Fig. 1). Data adapted from Bagdas et al. (2016).

acid-paired compartment is indicated in Fig. 4 as an "aversion score." Thus, the CR was avoidance of the acid-paired compartment. By contrast, mice treated with vehicle in both compartments did not change their preference.

Once parameters were established that reliably produced CPA as a CR, then drugs were tested for their effectiveness to block development of that CPA in separate groups of animals. Figure 4 shows that administration of morphine prior to acid-conditioning sessions dose-dependently blocked development of the acid-induced CPA, whereas treatment with the $\kappa$ agonist U50,488 did not. Importantly, both drugs were administered at doses sufficient to block the acidinduced UR (acid-stimulated stretching; see Fig. 2). The effectiveness of morphine to block both the acidinduced UR and acid-induced CPA is consistent with both 1) the conclusion that morphine blocked sensory sensitivity to acid as a noxious stimulus and 2) the clinical effectiveness of morphine as an analgesic in humans. By contrast, the failure of U50,488 to block acid-induced CPA suggests that U50,488 did not block sensory sensitivity to the acid noxious stimulus; this again is consistent with the conclusion that U50,488 decreased acid-stimulated stretching in Fig. 2 by producing motor impairment and with the failure of centrally acting $\kappa$ agonists as effective analgesics in humans.

This study also included another important control by evaluating effects of both drugs in mice that received only acid vehicle in both compartments during conditioning sessions. Thus, these mice received only vehicle in one compartment and only morphine or U50,488 in the other compartment during conditioning. Test sessions then provided an opportunity to evaluate conditioning effects of the drugs alone in the absence of the noxious stimulus. A high dose of $3.2 \mathrm{mg} / \mathrm{kg}$ morphine increased preference for the drug-paired compartment. This type of effect is known as a conditioned place preference (CPP), and it suggests two conclusions. First, this finding raises the possibility that morphineinduced blockade of the acid-induced CPA could have reflected a nonselective increase in preference for the morphine-paired compartment rather than a blockade of sensitivity to the acid stimulus; however, the 10-fold higher potency of morphine to block acid-induced CPA than to produce a CPP in the absence of acid argues against this possibility. Second, drug-induced CPP is often associated with abuse liability, and in this case, the effectiveness of morphine to produce a CPP is consistent with the known abuse liability of morphine (Tzschentke, 2007). In contrast to morphine, U50,488 had no effect when tested in the absence of the acid noxious stimulus. Notably, U50,488 and other centrally acting $\kappa$ agonists can produce CPA when administered alone in place-conditioning procedures (Shippenberg and Herz, 1986; Ehrich et al., 2015), but the single U50,488 conditioning session used in this study was not sufficient to produce a CPA. Accordingly, the failure of U50,488 to block acid-induced CPA in this study cannot be attributed to aversive effects of U50,488 alone that might have obscured any antinociceptive effects.

Table 3 summarizes effects of this and other studies that have examined effects of morphine in assays of pain-related CPA. Morphine has consistently been found to block pain-related CPAs at doses that failed to produce a CPP in the absence of the pain state. In the only exception shown in Table 3, continuous morphine treatment of 24 hours (via a subcutaneously implanted morphine pellet) failed to block movement-induced CPA in a model of "break-through" bone cancer pain, but a relevant methodological distinction of this study was 
TABLE 3

Effects of $\mu$-opioid agonist analgesics (e.g., morphine) in assays of pain-related CPA

\begin{tabular}{|c|c|c|c|c|c|c|c|}
\hline \multirow{2}{*}{ Subject } & \multirow{2}{*}{$\begin{array}{l}\text { Pain Stimulus (Time before } \\
\text { Conditioning) }\end{array}$} & \multicolumn{2}{|c|}{$\begin{array}{l}\text { Conditioning } \\
\text { Sessions }\end{array}$} & \multicolumn{2}{|c|}{ Morphine } & \multirow{2}{*}{ Result } & \multirow{2}{*}{ Reference } \\
\hline & & $\#$ & Duration & Dose (Route) & Time & & \\
\hline & & & $\min$ & $m g / k g$ & $\min$ & & \\
\hline Rats & $\begin{array}{l}\text { Spinal nerve ligation }(-3 \mathrm{wk})+ \\
\text { von Frey }(1 / \mathrm{min} \times 15 \mathrm{~min})\end{array}$ & 10 & 20 (2/day) & $0.3,3$ (s.c.) & -45 & Blocked CPA without $\mathrm{CPP}^{a}$ & Hummel et al., 2008 \\
\hline Rats & $\begin{array}{l}\text { Intraplantar carrageenan }(-4 \mathrm{~h})+ \\
\quad \text { von Frey }(1 / \mathrm{min} \times 15 \mathrm{~min})\end{array}$ & 2 & 20 (2/day) & $0.3,3$ (s.c.) & -45 & Blocked CPA without $\mathrm{CPP}^{a}$ & Hummel et al., 2008 \\
\hline Male SD rats & Intraplantar carrageenan $(-4 \mathrm{~h})$ & 2 & 40 & $\begin{array}{l}0.005-10 \\
\text { (i.p.) }\end{array}$ & -15 & $\begin{array}{l}\text { Blocked CPA; less potent } \\
\text { to produce } \mathrm{CPP}^{a}\end{array}$ & van der Kam et al., 2008 \\
\hline Male SD rats & Intraplantar CFA ( $-2 \mathrm{~h})$ & 2 & 30 (2/day) & $0.5-1.0$ (i.p.) & -30 & Blocked CPA without $\mathrm{CPP}^{a}$ & Zhang et al., 2012 \\
\hline Male ICR mice & Intraperitoneal acid (0 $\mathrm{min})$ & 2 & 40 (2/day) & $0.1-3.2$ (s.c.) & -15 & $\begin{array}{l}\text { Blocked CPA; less potent } \\
\text { to produce } \mathrm{CPP}^{a}\end{array}$ & Bagdas et al., 2016 \\
\hline $\begin{array}{l}\text { Male/female } \\
\text { SD rats }\end{array}$ & Intraplantar formalin (0 $\mathrm{min})$ & 2 & 45 & $0.5-4.0$ (s.c.) & -15 & Blocked CPA without $\mathrm{CPP}^{a}$ & Harton et al., 2017 \\
\hline $\begin{array}{l}\text { Male/female } \\
\text { Fischer } 344 \\
\text { rats }\end{array}$ & $\begin{array}{c}\text { Bone cancer }(-11 \text { days })+ \\
\text { leg movement }(0 \mathrm{~min})\end{array}$ & 2 & 30 (2/day) & $\begin{array}{l}\text { 75-mg } \\
\text { pellet } \\
\text { (s.c.) }\end{array}$ & -1 day & Did not block CPA & Havelin et al., 2017 \\
\hline Male SD rats & Intraplantar formalin (0 min) & 2 & 60 (2/day) & $\begin{array}{l}\text { 1, } 10 \mathrm{nmol} \\
\text { (vBNST) }\end{array}$ & $-10 \min$ & Blocked CPA without $\mathrm{CPP}^{a}$ & Maruyama et al., 2018 \\
\hline
\end{tabular}

ICR, Institute of Cancer Research; SD, Sprague-Dawley; vBNST, ventral bed nucleus of the stria terminalis.

${ }^{a}$ These results indicate an antinociceptive drug profile.

the sustained presence of morphine during conditioning in both compartments as well as during testing rather than during the conditioning sessions only (Havelin et al., 2017). Other $\mu$ agonists have also been found to block pain-related CPA (Rutten et al., 2014a), and in assays that used an inflammatory pain stimulus, NSAIDs were also effective to block pain-related CPAs without producing a CPP in the absence of the pain state (Rutten et al., 2014a; Bagdas et al., 2016). Taken together with data summarized above to show that the $\kappa$ agonist U50,488 failed to block a pain-related CPA (Bagdas et al., 2016), these data suggest that CPA procedures are both sensitive and selective for detecting analgesic drugs; however, this conclusion should be considered preliminary, given the limited number of published studies and limited range of tested drugs. Figure 5 provides a schematic to show the four different types of treatment groups that are typically required in a CPA study, together with the different types of effects that are possible with each treatment and how those treatment profiles can be interpreted.

"Fear-conditioning" and "conditioned-taste-aversion" procedures represent other categories of classical conditioning procedures that engender classically conditioned avoidance learning and that can be used in preclinical pain research (Curzon et al., 2009; Lin et al., 2017). In fear conditioning, the CR is typically an arrest of movement or "freezing" response (i.e., avoidance of locomotion), whereas in taste aversion, the CR is usually reduced consumption of a liquid with a distinctive taste. Both fear-conditioning and conditioned-taste-aversion procedures commonly use non-noxious aversive stimuli as the US, but noxious stimuli can also be used. In an example of a fear-conditioning study using a noxious stimulus as the US (Westbrook et al., 1991), rats were individually transported to a testing room in a plastic bucket and held in the bucket for 30 minutes before placement for 30 seconds into a test chamber with a copper floor that could be heated to various temperatures. The visual and other stimuli inherent to the bucket and test chamber served as the CS, and noxious floor temperatures $\left(50,52\right.$, or $\left.54^{\circ} \mathrm{C}\right)$ served as the PS sufficient to elicit paw licking as the UR indicative of nociception. Conditioning consisted of one pairing of CS + PS. Testing occurred 1 day later by again exposing rats to the CS (i.e., 30 minutes in the bucket, followed by placement in the chamber); however, on the test day, the floor was not heated, rats were placed on a small platform above the floor, and the latency to step down off the platform onto the unheated floor was measured. Rats conditioned with CS+PS displayed longer stepdown latencies than control rats. Thus, the CR was a delayed step-down response interpreted as evidence of "fear." The effects of both morphine and the GABA $_{A}$ receptor positive allosteric modulator diazepam were evaluated on development of this "fear" CR by administering morphine or diazepam prior to the conditioning trial. During conditioning, morphine pretreatment decreased the heat-induced paw-licking UR, whereas diazepam did not. However, during testing, the delayed step-down CR was reduced for both drugs (i.e., rats treated with either morphine or diazepam during conditioning stepped down more quickly than rats treated with vehicle during conditioning). This profile of results was interpreted to suggest that morphine blocked conditioning by blocking sensitivity to the thermal PS (i.e., antinociception), whereas diazepam did not block thermal sensitivity but produced learning impairment that blocked consolidation of the CS+US association.

Studies of classical conditioning with noxious stimuli as the US have two principal advantages for analgesic 
A

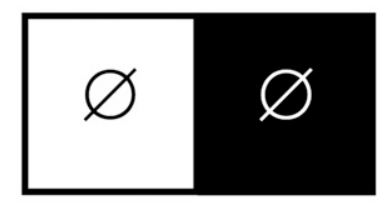

B
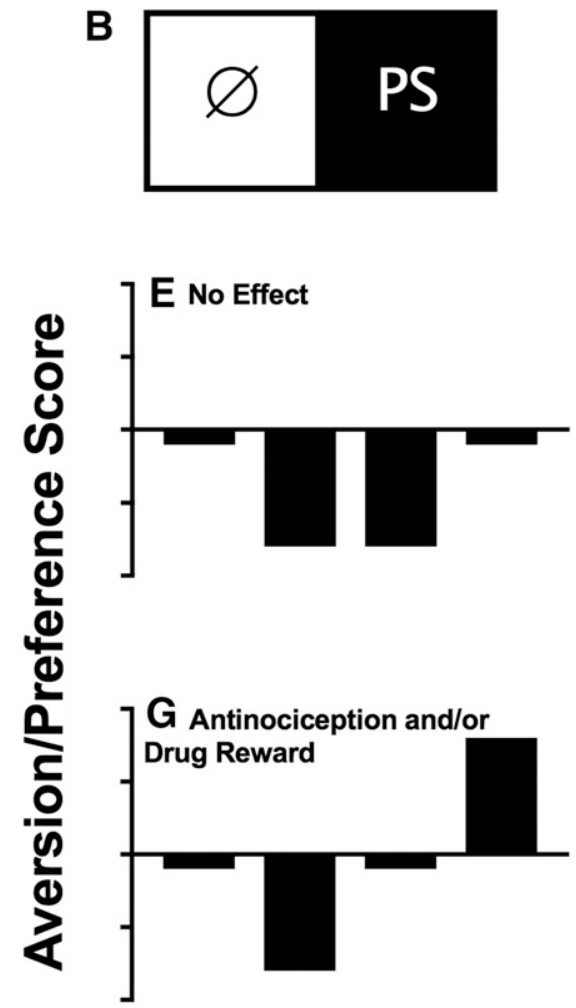

C

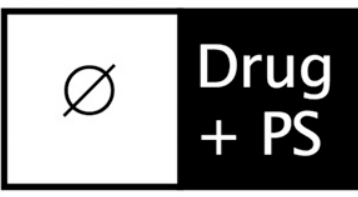

D

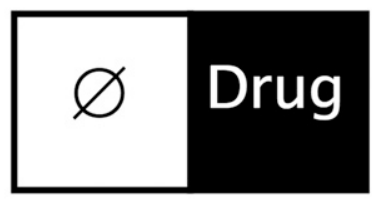

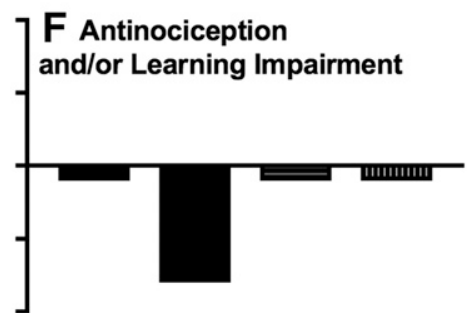

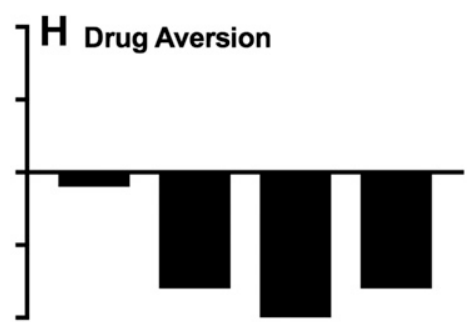

A $B$ C D

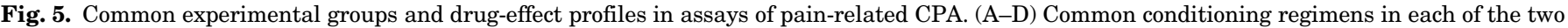

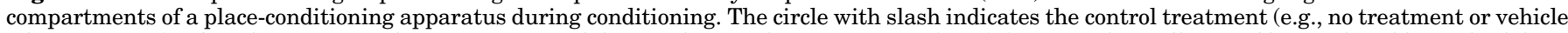

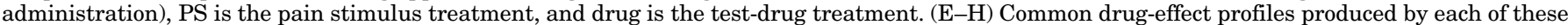

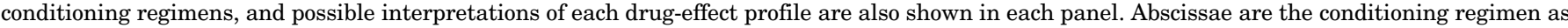

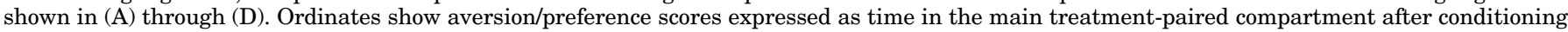
minus time in that compartment before conditioning. Bars below and above the abscissa indicate a CPA or CPP, respectively.

drug development. First, from the perspective of preclinical drug assessment, test drug effects can be evaluated on both 1) the noxious stimulus-induced UR during conditioning sessions when the drug is present and 2) the CR during the subsequent test session when the drug is absent. An effective analgesic that blocks sensory sensitivity to the noxious stimulus (i.e., a true analgesic like morphine) will decrease both the UR and the CR. By contrast, blockade of the UR but not the CR (e.g., U50,488 in the CPA experiment described above; Bagdas et al., 2016) suggests a nonanalgesic effect such as motor impairment. Reciprocally, blockade of the CR but not the UR (e.g., diazepam in the fear-conditioning experiment described above; Westbrook et al., 1991) suggests learning impairment.

A second advantage of research on classically conditioned pain behaviors is that they rely on supraspinal neural circuits distinct from the spinal and brainstem neural circuits that mediate many unconditioned pain-stimulated behaviors. For example, the first study to use a pain-related CPA procedure evaluated the effects not of an analgesic drug but of lesions to the rostral anterior cingulate cortex, a brain region thought to be important in processing "affective" as opposed to "sensory" dimensions of pain (Johansen et al., 2001). In this study, intraplantar injection of formalin served as the PS both to elicit paw licking and flinching behavior as a UR and to produce CPA as a CR. Lesions to the rostral anterior cingulate cortex failed to block the paw licking/flinching UR but did block development of the CPA CR. These results were interpreted to suggest that the rostral anterior cingulate cortex is necessary for either detection or learning of the "aversiveness" of the formalin stimulus but not for the paw licking/flinching reflexive responses. A series of more recent studies has elaborated on the supraspinal neural circuits that underlie pain-related CPA (Tanimoto et al., 2003; Deyama et al., 2007, 2008; Wang et al., 2007) and demonstrated 
that morphine microinjection into the bed nucleus of the stria terminalis was also sufficient to block formalininduced CPA without blocking formalin-induced paw licking/flinching (Maruyama et al., 2018). Taken together, these results provide further evidence that different pain behaviors can have different neural mechanisms and respond differentially to pharmacological treatments.

A final advantage of classical conditioning procedures is that classical conditioning plays a role in shaping the human experience of pain (Jensen et al., 2015; Madden et al., 2016; Harvie et al., 2017), and determinants of the development, expression, and pharmacological modulation of these classical conditioning effects can be examined in preclinical studies. A detailed discussion of the literature on this topic is beyond the scope of this review, but the basic principle is that human experience often includes concurrent exposure to initially neutral stimuli in conjunction with noxious stimuli (e.g., the appearance and smell of a medical facility paired with a painful surgical procedure). By the principles of classical conditioning, these neutral stimuli can come to function as CSs producing painrelated CRs that are either unpleasant in their own right (e.g., anxiety, fear) or that complicate further treatment (e.g., avoidance of the medical facility where treatment is provided). In this regard, drugs such as diazepam may have utility in clinical pain management not because they block sensitivity to noxious stimuli, but because they block conditioned associations between those stimuli and other neutral stimuli in the environment, thereby attenuating development of undesirable CRs.

Two weaknesses will be mentioned for studies that use a noxious stimulus as the US in classical conditioning. First, as noted above, test drugs can block the formation of a pain-related CR not only by blocking sensory sensitivity to the US, but also by blocking sensitivity to the CS or by impairing the formation of new memories. Blockade of sensitivity to the CS is likely a minor issue in most studies of pain-related conditioning because the CS is often a compound stimulus with components in multiple stimulus modalities. For example, in place-conditioning studies, the CS often consists of both visual and tactile stimuli. Nonetheless, the potential for false-positive effects due to impaired sensitivity to the CS should be considered. The more significant risk of false-positive effects lies in treatments that impair learning, and although blockade of pain-related associations may have some clinical benefit, it is not the goal of most analgesic drug development programs. There are several strategies to evaluate the potential role of learning deficits in studies that examine drug effects on pain-related CRs. For example, van der Kam et al. (2008) found that morphine blocked development of a pain-related CPA when the morphine was administered before the PS during a conditioning session, but not when it was administered immediately after the conditioning session. This finding was interpreted to suggest that morphine blocked sensory sensitivity to the PS during conditioning, but it did not alter memory consolidation processes occurring in the period after the conditioning session. As another example, Bagdas et al. (2016) found that morphine blocked development of a painrelated CPA but not CPA to a non-noxious aversive stimulus (lithium chloride injection), suggesting that morphine produced a selective decrease in pain-related learning rather than a general impairment of all learning. Lastly, as noted above, drug effects can be compared on both initial expression of the PS-elicited UR during conditioning and on subsequent expression of the CR during testing. Clinically effective analgesics generally block both the UR and CR, whereas learning impairment should be considered if only the CR is blocked.

A second challenge in classical conditioning studies is optimal temporal alignment of the CS and PS during conditioning sessions. Alignment of CS and PS can be most precisely controlled with stimuli that have rapid onsets, short durations, and rapid offsets, and the optimal arrangement is usually a form of delay conditioning as shown in Fig. 3. However, pain stimuli often have relatively slow onsets, long durations, and variable offsets that can complicate conditioning. For example, many of the studies of pain-related CPA summarized in Table 3 used chemical stimuli (intraplantar formalin, intraplantar carrageenan, intraperitoneal acid), with onsets of seconds to minutes, durations of minutes to hours, and poorly defined offsets. However, no published studies have systematically examined the role of stimulus alignment in producing pain-related CPAs. For example, delay conditioning involves presentation of the CS for some period of time before and during the US, but in general, the studies in Table 3 delivered the PS immediately before confinement to the pain-paired compartment, so there was little if any explicit opportunity for CS exposure prior to PS onset. It is reasonable to suppose that longer CS exposure prior to PS delivery might produce either more robust CPA or CPA with lower PS intensities, but data to address this issue have not been published. Similarly, the duration of conditioning sessions for studies in Table 3 were 20-60 minutes, but the role of session duration as a determinant of painrelated CPA has also not yet been systematically studied. Temporal alignment of CS and PS is even more challenging with putative chronic pain states, such as in models of chronic inflammatory or neuropathic pain thought to produce pain lasting days, weeks, or months. In humans, chronic inflammatory and neuropathic states are often characterized by spontaneous pain (i.e., pain in the absence of other overt evocative stimuli), but the timing of spontaneous pain episodes cannot be precisely controlled in the 
laboratory and accordingly cannot be used as a reliable US in classical conditioning studies. Rather, it is necessary in CPA models of chronic pain to also incorporate an acute evocative stimulus that can be more precisely controlled. For example, Hummel et al. (2008) engendered CPA in rats treated with either an inflammatory or neuropathic insult hours or weeks, respectively, before any conditioning. As a result, any spontaneous pain associated with the inflammatory/neuropathic injury was present during conditioning in both compartments as well as during testing, and hence could not serve as the US. To produce CPA, these investigators supplemented the underlying inflammatory/neuropathic pain stimulus with mechanical stimulation (probing the hindpaws with a relatively high-force von Frey filament) that was paired with only one compartment. Accordingly, in this study, the US consisted of the inflammatory/neuropathic stimulus + acute mechanical stimulation, and CPA presumably depended on hypersensitive pain responses to mechanical stimulation. Similarly, Havelin et al. (2017) established a CPA in rats using a US consisting of an underlying model of bone cancer supplemented by subsequent acute mechanical stimulation (manual articulation of the affected hindlimb) that was paired with one compartment.

3. Pain Stimulus as a Contextual Stimulus. In a second type of classical conditioning study, the noxious "pain" stimulus functions as a contextual stimulus that modulates conditioning with other non-noxious stimuli. This role can be expressed as follows:

$$
\mathrm{PS} \rightarrow[\mathrm{CS}+\mathrm{US} ; \mathrm{CS} \rightarrow \mathrm{CR}]
$$

Although many permutations of this approach are possible, perhaps the most popular is guided by the supposition that just as pain onset can be aversive and produce a CPA, so pain offset can be rewarding and produce a CPP (Shippenberg et al., 1988; Sufka, 1994; King et al., 2009). Thus, in this type of experimental design, the PS serves as a contextual stimulus intended to produce an underlying pain state, and the US is a drug or other treatment hypothesized to terminate that pain state. For example, one study compared placeconditioning effects of intrathecal clonidine and intrathecal adenosine in rats with a spinal nerve ligation model of neuropathy (King et al., 2009). Ten days after nerve ligation or sham surgery, rats received two conditioning sessions, in which intrathecal saline was paired with one compartment of a place-conditioning apparatus, and intrathecal clonidine or adenosine was paired with the other compartment. During a test session the day after conditioning, rats received no treatments, and allocation of time between the two compartments was assessed. Intrathecal clonidine produced a CPP in the rats with nerve ligation but not in the sham-operated controls, whereas intrathecal adenosine failed to alter preferences in either group. These results were interpreted to suggest that nerve ligation produced a chronic and aversive neuropathic pain state that was alleviated by intrathecal administration of clonidine but not adenosine. These results were also considered to be consistent with clinical experience indicating that intrathecal clonidine, but not intrathecal adenosine, is an effective treatment of some types of neuropathic pain. Figure 6 provides a schematic to show the four different types of treatment groups that are typically required in a CPP study, together with the possible outcomes with test drugs and how those treatment outcomes can be interpreted.

The main strength of the CPP experimental design as a classical conditioning procedure is its potential for evaluating pharmacological relief of chronic spontaneous pain. As noted above, putative chronic pain states produced by manipulations such as inflammation or nerve injury are unwieldy as the US in classical conditioning procedures because the onset and duration of the pain state are protracted and potentially variable across subjects, and hence difficult to pair precisely with a CS. In CPP studies, the PS is thought to produce a sustained spontaneous pain state that does not require additional evocative stimuli and that is present during all conditioning sessions as well as during testing (see Fig. 6B). The US is not the pain stimulus, but rather a drug hypothesized to produce pain relief. The temporal parameters of these drug stimuli can be controlled as a US for pairing with a CS, and treatments that produce CPP with greater potency or effectiveness in injured animals than in controls provide evidence for both existence of the underlying spontaneous pain state and the relief of that pain state.

An additional strength of this type of procedure, as with all classical conditioning procedures, is that drug effects are evaluated by measuring a CR expressed when the drug is no longer present. As a result, drug effects on motor function (e.g., motor impairment) that might occur during conditioning sessions when drug is present are not expected to confound measurement of CR expression during subsequent test sessions when drug is absent. Also, as with CPA procedures described above, CPP procedures rely on supraspinal circuits thought to be important in the "affective" dimensions of pain, and CPP procedures have been used to dissociate neural circuits that mediate drug-induced CPP from circuits that mediate drug effects on pain-stimulated behaviors (King et al., 2009; Navratilova et al., 2015, 2016; Kang et al., 2017; Wang et al., 2017).

A significant weakness of CPP experimental designs is their unreliable sensitivity to clinically effective analgesics. For example, Table 4 summarizes studies that have examined morphine effects in CPP procedures. Although analgesic drugs are expected to produce CPP more potently and/or effectively in chronic 
A

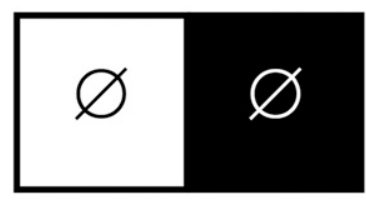

B
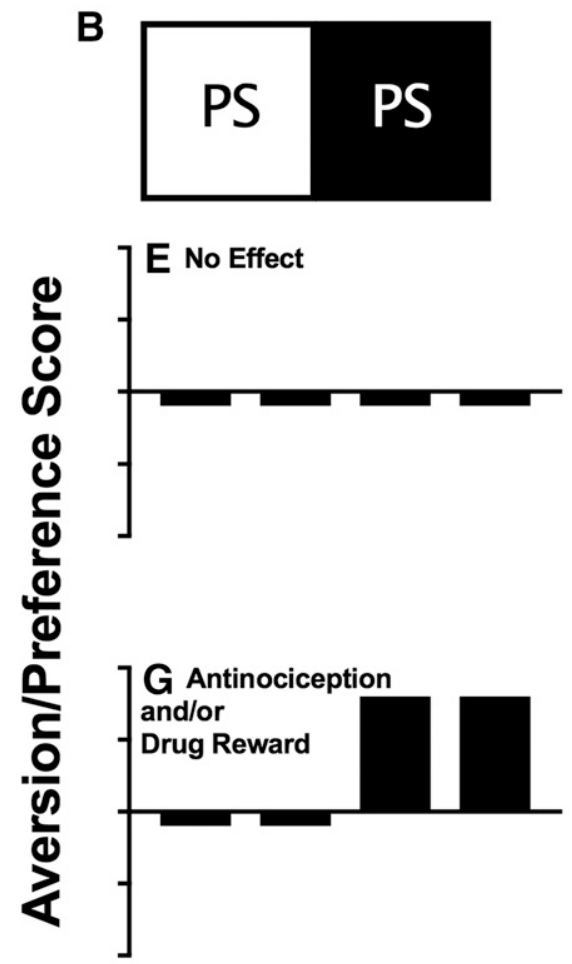

A $\quad$ B $\quad$ C $\quad$ D

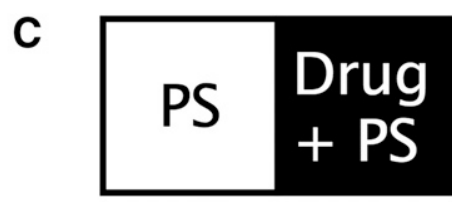

D
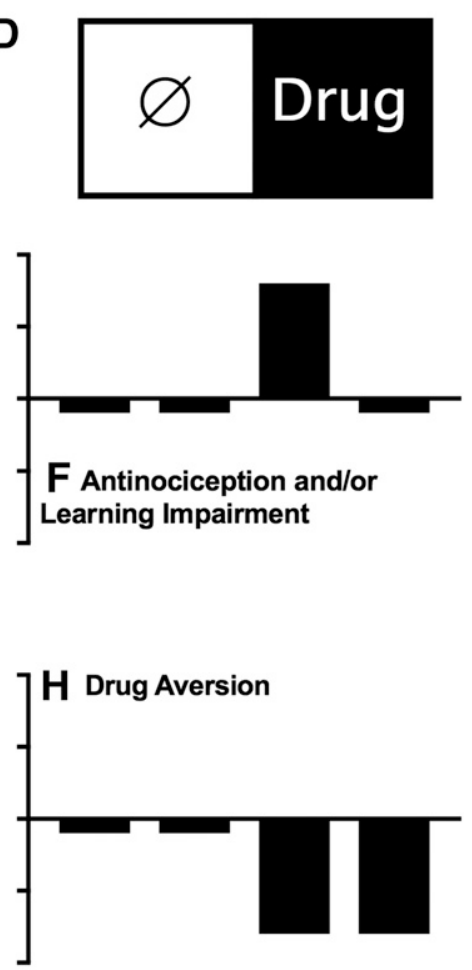

A $\quad$ B $\quad$ C $\quad$ D

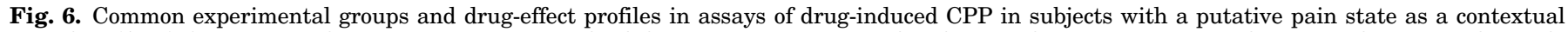

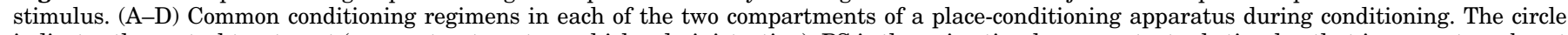

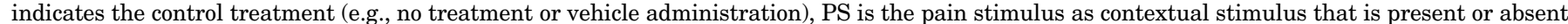

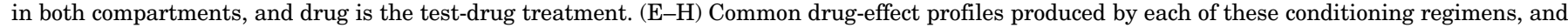

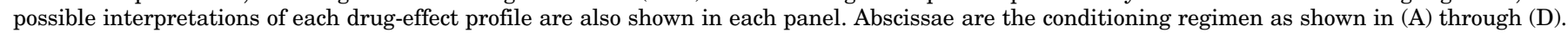

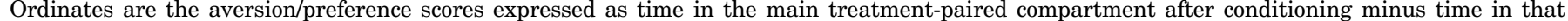
compartment before conditioning. Bars below and above the abscissa indicate a CPA or CPP, respectively.

pain states than in control states, morphine and other $\mu$ agonist analgesics (Nakamura et al., 2008; Mori et al., 2014) often fail to produce this profile. Thus, of the 18 experiments summarized in Table 4 using a range of different chronic pain manipulations, morphine produced a clear antinociceptive profile in only six experiments; in many studies, the pain manipulations decreased morphine CPP relative to that observed in control subjects. Similarly, NSAIDs also usually fail to produce CPP in subjects with presumed chronic inflammatory pain (Shippenberg et al., 1988; Sufka, 1994; Suzuki et al., 1996; Park et al., 2016), although CPP is sometimes observed (e.g., in a $\mathrm{K} / \mathrm{BxN}$ serum transfer model of rheumatoid arthritis; Park et al., 2016). The poor sensitivity of these procedures to detect known analgesics obviously raises concern about their utility for evaluating novel compounds.

The reasons for this poor sensitivity remain to be determined, but one factor may be the dependence of classical conditioning on temporal alignment of the US and CS. Table 4 suggests that morphine has been most effective to produce an antinociceptive CPP profile when relatively low doses are administered by a route of administration that produces rapid onset of drug effects (i.e., intravenous or intracranial), and conditioning sessions are relatively short. However, Table 4 also shows exceptions to this hypothesis. More systematic parametric studies would be required to clarify the importance of these variables for morphine effects, and the prospect of addressing these issues with every new test drug is daunting.

An alternative approach has been to establish an "analgesia-selective" CPP using some other treatment, and then to evaluate the effectiveness of a test drug to block CPP by that treatment. In one study, for example, peripheral nerve block with the local anesthetic lidocaine produced CPP in rats with a model of bone cancer pain but not in control rats (Remeniuk et al., 2015). This lidocaine-induced CPP was blocked by systemic morphine (a 75-mg pellet implanted subcutaneously the day 
TABLE 4

Effects of morphine in assays of analgesia-related CPP

\begin{tabular}{|c|c|c|c|c|c|c|c|}
\hline \multirow{2}{*}{ Subjects } & \multirow{2}{*}{$\begin{array}{l}\text { Pain Stimulus (Time before } \\
\text { Conditioning) }\end{array}$} & \multicolumn{2}{|c|}{$\begin{array}{l}\text { Conditioning } \\
\text { Sessions }\end{array}$} & \multicolumn{2}{|c|}{ Morphine } & \multirow{2}{*}{ Result } & \multirow{2}{*}{ Reference } \\
\hline & & $\#$ & Duration & Dose (Route) & Time & & \\
\hline & & & $\min$ & $m g / k g$ & $\min$ & & \\
\hline Male SD rats & Intraplantar CFA & 6 & 60 & $\begin{array}{l}0.3-5.6 \\
\text { (s.c.) }\end{array}$ & -0 & $\mathrm{CPP}$ in pain $=$ nonpain & Shippenberg et al., 1988 \\
\hline Male SD rats & Intraplantar CFA ( -1 day) & 8 & 60 & 3-10 (i.p.) & -0 & $\mathrm{CPP}$ in pain $=$ nonpain & Sufka, 1994 \\
\hline Male SD rats & $\begin{array}{l}\text { Intraplantar carrageenan ( }-1 \text { day), } \\
\text { intraplantar formalin ( }-1 \text { day) }\end{array}$ & 4 & 50 & $2-8$ (i.p.) & -0 & $\begin{array}{l}\text { No CPP in pain, CPP in } \\
\text { nonpain }\end{array}$ & Suzuki et al., 1996 \\
\hline Male SD rats & Partial nerve ligation ( -4 days) & 6 & 60 & $4-8$ (s.c.) & -0 & $\begin{array}{l}\text { No CPP in pain, CPP in } \\
\text { nonpain }\end{array}$ & Ozaki et al., 2002 \\
\hline $\begin{array}{l}\text { Male C57Bl/6J } \\
\text { mice }\end{array}$ & Partial nerve ligation ( -4 days) & 6 & 30 (2/day) & $2.5-5$ (s.c.) & -0 & $\begin{array}{l}\text { No CPP in pain, CPP in } \\
\text { nonpain }\end{array}$ & Petraschka et al., 2007 \\
\hline \multirow{3}{*}{$\begin{array}{l}\text { Female C57Bl/6J } \\
\text { mice }\end{array}$} & Intraplantar carrageenan $(-4 \mathrm{~h})$ & 6 & 20 (2/day) & 10 (i.p.) & -5 & CPP in pain = nonpain & Betourne et al., 2008 \\
\hline & Intraplantar CFA ( $-4 \mathrm{~h})$ & 6 & 20 (2/day) & 10 (i.p.) & -5 & $\begin{array}{l}\text { No } \mathrm{CPP} \text { in pain, } \mathrm{CPP} \text { in } \\
\text { nonpain }\end{array}$ & Betourne et al., 2008 \\
\hline & Intraplantar tumor cells ( -20 days) & 6 & 20 (2/day) & 10 (i.p.) & -5 & $\begin{array}{l}\text { No CPP in pain, CPP in } \\
\text { nonpain }\end{array}$ & Betourne et al., 2008 \\
\hline $\begin{array}{l}\text { Male/female } \mathrm{Tg} \\
\text { mice }\end{array}$ & Partial nerve ligation ( -3 days) & 6 & 60 & 5 (s.c.) & -0 & $\mathrm{CPP}$ in pain $<$ nonpain & Niikura et al., 2008 \\
\hline Male SD rats & Spinal cord contusion ( -2 days) & 4 & 45 (2/day) & $\begin{array}{l}1.25,2.5 \\
(?)\end{array}$ & -0 & CPP in pain $\geq$ nonpain $^{a}$ & Woller et al., 2012 \\
\hline Male SD rats & $\begin{array}{l}\text { Paclitaxel treatment ( }-7 \text { days }) \\
\text { Oxaliplatin treatment ( }-7 \text { days })\end{array}$ & 6 & 60 & 4 (s.c.) & -0 & $\mathrm{CPP}$ in pain $\leq$ nonpain & Mori et al., 2014 \\
\hline Female Tg mice & Intraplantar CFA ( -3 days) & 6 & 30 (2/day) & 0.1 (i.p.) & -0 & CPP in pain $\geq$ nonpain $^{a}$ & Zhang et al., 2014 \\
\hline \multirow[t]{3}{*}{ Male SD rats } & Spinal nerve ligation ( -21 to -14 days) & 2 & 30 (2/day) & $\begin{array}{l}0.5,4.0 \\
\text { (i.v.) }\end{array}$ & -0 & $\mathrm{CPP}$ in pain $\geq$ nonpain $^{a}$ & Navratilova et al., 2015 \\
\hline & Spinal nerve ligation ( -21 to -14 days) & 2 & 30 (2/day) & $20 \mu \mathrm{g} / \mathrm{side}$ & -0 & CPP in pain $\geq$ nonpain $^{a}$ & Navratilova et al., 2015 \\
\hline & Paw incision ( -1 day) & 2 & 30 (2/day) & $\begin{array}{c}20 \mu \mathrm{g} / \mathrm{side} \\
(\mathrm{rACC})\end{array}$ & -0 & CPP in pain $\geq$ nonpain $^{a}$ & Navratilova et al., 2015 \\
\hline $\begin{array}{c}\text { Male/female } \\
\text { C57BI/6 } \\
\text { mice }\end{array}$ & Paclitaxel treatment ( -3 days) & 6 & 30 & $\begin{array}{l}0.3-10 \\
\text { (i.p.) }\end{array}$ & -15 & $\mathrm{CPP}$ in pain $=$ nonpain & Neelakantan et al., 2016 \\
\hline Male LE rats & Peripheral nerve injury ( -6 days) & 8 & 30 & $1-8$ (s.c.) & -0 & CPP in pain $\geq$ nonpain $^{a}$ & Cahill et al., 2013 \\
\hline \multirow[t]{2}{*}{$\begin{array}{l}\text { Male/female } \\
\text { SD rats }\end{array}$} & Intraplantar CFA ( -1 days $)$ & 2 & 45 & $1-12$ (s.c.) & -0 & $\mathrm{CPP}$ in pain $\leq$ nonpain & $\begin{array}{l}\text { Armendariz and Nazarian, } \\
2018\end{array}$ \\
\hline & Intraplantar CFA ( -7 days) & 2 & 45 & $1-8$ (s.c.) & -0 & $\mathrm{CPP}$ in pain $>$ nonpain $^{a}$ & $\begin{array}{l}\text { Armendariz and Nazarian, } \\
2018\end{array}$ \\
\hline
\end{tabular}

LE, Long-Evans; rACC, rostral anterior cingulate cortex; SD, Sprague-Dawley; Tg, transgenic.

${ }^{a}$ These results indicate an antinociceptive drug profile.

before conditioning), and this finding was interpreted to suggest that morphine produced a sustained analgesia that blocked effectiveness of lidocaine to produce any additional analgesia that might serve as a US and maintain CPP. However, this approach is logistically complicated insofar as it requires a chronic pain state (and controls), a treatment sufficient to produce reliable CPP in that pain state (and controls), and administration of the test drug during all conditioning sessions (i.e., in both compartments during conditioning). Moreover, interpretation of drug effects produced by novel compounds can be complicated because CPP induced by the effective treatment can be blocked not only by analgesia to overshadow effects of that treatment but also by manipulations that block its antinociceptive mechanisms. For example, peripheral nerve block-induced CPP in rats with either paw-incision or spinal nerve ligation injuries could be blocked by administration of the opioid antagonist naloxone into the rostral anterior cingulate cortex (Navratilova et al., 2015). The interpretation here was not that naloxone produced analgesia that overshadowed effects of the peripheral nerve block; rather, the results were interpreted to suggest that naloxone blocked opioid receptor signaling that mediated effects of the nerve block.

A second weakness of CPP procedures in preclinical pain research is the absence of direct evidence for existence of the pain state independent of drug effects. The value of CPP procedures lies in their intended utility to evaluate sustained spontaneous pain states thought to result from inflammatory, neuropathic, or other chronic pain manipulations. However, as noted above, many manipulations intended to produce chronic pain turn out to have little or no effect on spontaneous behaviors (e.g., little or no evidence of unconditioned pain-stimulated or pain-depressed behavior). As a result, in contrast to CPA procedures that use noxious stimuli with clear URs, there is little opportunity in CPP procedures to compare drug effects on pain-related URs with pain-related CRs. As an alternative, many studies compare drug-induced CPP effects with drug effects on hypersensitive URs elicited by evocative stimuli such as von Frey filaments; however, this is an apples-to-oranges comparison of drug effects on putative manifestations of spontaneous versus evoked pain, which are considered a priori to be distinct (e.g., Remeniuk et al., 2015; 
Havelin et al., 2017). Additionally, the possibility also exists that any aversive state produced by putative "chronic pain" insults may reflect sensations other than pain such as nonpainful dysesthesias (e.g., tingling, itch), and drug-induced CPP may reflect relief of those aversive but nonpainful states rather than relief of pain. For example, the effectiveness of gabapentin to produce CPP in rodents with neuropathic insults (Park et al., 2013; Bannister et al., 2017) is not consistent with its relatively weak effectiveness to treat neuropathic pain (Pachman et al., 2011; Finnerup et al., 2015), but may be related to its effectiveness to treat neuropathy-associated itch (Maciel et al., 2014). Moreover, the poor sensitivity of CPP procedures to $\mu$ agonists and NSAID analgesics suggests that the underlying aversive state may be something other than pain.

A final weakness of CPP procedures, as with CPA procedures, is their vulnerability to false-positive effects with treatments that impair learning. For example, Sufka (1994) found that neither morphine nor indomethacin produced an antinociceptive CPP profile in rats treated with intraplantar CFA to induce a putative inflammatory pain state; however the $N$-methyl-D-aspartate (NMDA) glutamate receptor antagonist $(5 R, 10 S)-(+)-5$-methyl10,11-dihydro-5H-dibenzo[ $a, d]$ cyclohepten-5,10-imine (MK-801) did produce a CPP. ${ }^{1}$ This result was interpreted as evidence for pain relief by MK-801; however, MK-801 and other NMDA receptor antagonists are also well known to impair some types of neural plasticity and learning (Morris et al., 1990; Neill et al., 2010), and as a result, MK-801-induced CPP may also have reflected learning impairment.

4. Summary. In summary, the primary application of classical conditioning procedures to preclinical analgesic drug development has involved place-conditioning procedures to study either 1) drug effects on painrelated place aversion or 2 ) drug-induced conditioned place preferences in the context of a putative pain state. However, other classical conditioning procedures (e.g., fear-conditioning and conditioned-tasteaversion procedures) can also be used. Before concluding the section on classical conditioning in preclinical pain research, three other points warrant mention. First, it is

\footnotetext{
1 In the study by Sufka (1994), morphine did not produce differential CPP in rats with versus without the pain manipulation (intraplantar CFA) as assessed by a conventional measure of time spent in the drug-paired compartment. It did, however, produce selective CPP in intraplantar CFA rats by a different measure involving choice of the drug-paired compartment during repeated discrete-trial choice opportunities. This discrete-trial method of evaluating place preference was recommended by the author for use in place-conditioning studies of antinociception, but surprisingly, this approach was not used in a more recent study by this author (Roughan et al., 2014), nor has it been adopted by others. Moreover, indomethacin failed to produce CPP in intraplantar CFA rats by either measure (Sufka, 1994).
}

theoretically possible for the pain stimulus to play the role of $\mathrm{CS}$ in a classical conditioning experimental design. This role would be designated as follows: 1) PS $+\mathrm{US}$; 2) PS $\rightarrow$ CR. Although this type of design has not been used in preclinical studies, possible examples in human pain experience might include the potential for negative associations between pain stimuli and other aversive events, such as unemployment. In these cases, pain stimuli may elicit conditioned responses of exaggerated anxiety or fear, and analgesics may not eliminate all features of the pain stimulus that elicit negative conditioned responses. There is also potential for positive associations between noxious stimuli and rewarding stimuli that can occur during exercise (e.g., when athletic success serves as the US), intravenous drug abuse with a hypodermic needle (e.g., where the US is the effect of the abused drug), or masochism (e.g., where the US is sexual activity). Pain stimuli participating as the CS in positive associations may produce a conditioned response of pleasure rather than pain, and analgesic drugs would not be used to reduce such conditioned responses.

Second, it is also possible to use noxious stimuli as contextual stimuli in studies to examine pain effects on learning and cognitive function. Clinical pain states have been associated with various cognitive deficits due, for example, to allocation of attention toward pain sensations and away from other external or internal stimuli that might normally be detected and integrated into new learning (Terrando et al., 2011; Mazza et al., 2018). For example, one study evaluated the effects of paw incision in a model of fear conditioning in mice (Zhang et al., 2013). Mice were initially given either a paw-incision or sham surgery to produce a sustained pain state as a contextual stimulus. Twenty-four hours later, mice were exposed to a classical conditioning regimen in which a tone CS was paired with a brief foot shock US. During testing the next day, the CS alone produced "freezing behavior" as the CR, and expression of this CR was attenuated in the mice with paw incision. This finding supported other evidence to suggest that paw incision produced a hippocampus-independent learning impairment associated with reduced NMDA receptor expression in the prefrontal cortex. Analgesic treatment before the conditioning would presumably prevent this pain-related learning impairment, but these types of experimental designs have not yet been used to evaluate effects of candidate analgesic drugs.

Lastly, classical conditioning of analgesic responses can contribute to clinical treatment responses. For example, CSs paired with analgesic drug delivery can come to elicit an analgesic CR in the absence of the analgesic drug (i.e., the "placebo response"; Price et al., 2008; Greene et al., 2009; Babel et al., 2018). The determinants of classical conditioning effects and their interactions with candidate analgesic drugs can be examined in preclinical procedures that use pain 
stimuli as a contextual stimulus and analgesic drugs as the US (Benedetti, 2012; McNabb et al., 2014).

\section{Operant Conditioning}

1. Definition. Operant conditioning is a type of learning in which behavior is shaped by its consequences according to a "three-term contingency" (Skinner, 1974; Pierce and Cheney, 2017). The arrangement of these three terms can be diagrammed as follows:

$$
\mathrm{S}^{\mathrm{D}} \rightarrow \mathrm{R} \rightarrow \mathrm{S}^{\mathrm{C}}
$$

where $\mathrm{S}^{\mathrm{D}}$ denotes a discriminative stimulus, $\mathrm{R}$ denotes a behavioral response by the subject, and $\mathrm{S}^{\mathrm{C}}$ denotes a consequent stimulus delivered to the subject as a consequence of its behavior. In a preclinical example of operant conditioning, a mouse or rat can be placed into a chamber equipped with stimulus lights, a response lever, and a trough where food pellets can be delivered. Contingencies can then be established such that when the stimulus lights are illuminated (the $\mathrm{S}^{\mathrm{D}}$ ), then performance of lever-pressing behavior (the $R$ ) results in delivery of a food pellet (the $\mathrm{S}^{\mathrm{C}}$ ), whereas when the stimulus lights are off, then lever-pressing does not produce food delivery. Under these conditions, subjects will generally learn to respond on the lever when the stimulus lights are illuminated but not when the lights are off.

Operant conditioning plays a major role in shaping the behavior of humans as well as laboratory animals. In an example of human behavior analogous to the animal behavior described above, illumination of the distinctive sign of a fast-food restaurant (the $\mathrm{S}^{\mathrm{D}}$ ) indicates that performance of a behavioral response (placing and paying for an order at the counter, the $\mathrm{R}$ ) will result in delivery of the ordered food (the $\mathrm{S}^{\mathrm{C}}$ ). Operant conditioning is also involved in the acquisition and maintenance of many other behaviors as diverse as speaking a language, driving a car, or seeking treatment for an injury. In general terms, the probability of a behavior increases if it produces positive outcomes and decreases if it produces negative outcomes. Stated more formally, consequent stimuli (like food delivery in the examples above) that increase the probability of the preceding behavior are considered to function as "reinforcers," whereas consequent stimuli that decrease the probability of the preceding behavior are considered to function as "punishers."

In both humans and in preclinical studies using operant-conditioning procedures in animals, pain stimuli can serve in one of three different stimulus roles: 1 ) as the $\mathrm{S}^{\mathrm{D}}, 2$ ) as the $\mathrm{S}^{\mathrm{C}}$, or 3 ) as a contextual stimulus that modifies behavior maintained by contingencies involving other types of $\mathrm{S}^{\mathrm{D}}$ and $\mathrm{S}^{\mathrm{C}}$. Test drugs can then be evaluated for their effectiveness to block either the discriminative-, consequent-, or contextual-stimulus effects of the pain stimulus.
2. Pain Stimulus as the Discriminative Stimulus. Perhaps the most intriguing and misunderstood role of pain stimuli in operant conditioning is in their role as a discriminative stimulus according to the following relationship:

$$
\mathrm{PS} \rightarrow \mathrm{R} \rightarrow \mathrm{S}^{\mathrm{C}}
$$

where PS again denotes the pain stimulus. In this role, the presence of the pain stimulus PS signals that performance of behavioral response $R$ will produce a consequent stimulus $\mathrm{S}^{\mathrm{C}}$ that typically functions as a reinforcer. Conversely, when the PS is absent, then $R$ does not produce $S^{C}$. The use of a pain stimulus as the $\mathrm{S}^{\mathrm{D}}$ in preclinical research is exceedingly rare and may be unfamiliar to many preclinical researchers; however, as will be discussed in more detail below, this is by far the most common role of pain stimuli in human pain assessment. This massive discordance between preclinical and clinical studies in their focus on pain discrimination is a major obstacle to effective translational research.

Discrimination procedures are commonly used in laboratory animals to evaluate perception of nonpain stimuli. For example, in drug-discrimination procedures, a drug of interest serves as the discriminative stimulus, and drug discrimination in animals correlates well with the subjective (i.e., verbally described) effects of drugs in humans (Overton, 1988; Schuster and Johanson, 1988; Ator and Griffiths, 2003; Carter and Griffiths, 2009). In one of the rare preclinical examples of noxious-stimulus discrimination, rhesus monkeys responded during daily operant behavioral sessions consisting of discrete trials (Thomas et al., 1992). Each trial began when the monkey pressed and held a response key. Beginning at a variable time (3-9 seconds) after trial initiation, a thermode attached to the subject's upper lip delivered a noxious heat stimulus consisting of a rapid increase in temperature of $0.4,0.6$, or $1.0^{\circ} \mathrm{C}$ from a baseline of $46^{\circ} \mathrm{C}$. Release of the response key within 2.4 seconds of the heat stimulus produced juice delivery. Thus, in this procedure, the increase in thermode temperature served as the painrelated $\mathrm{S}^{\mathrm{D}}$, release of the response key within 2.4 seconds served as the $R$, and juice delivery served as the $\mathrm{S}^{\mathrm{C}}$. If the response occurred in the absence of the $\mathrm{S}^{\mathrm{D}}$ (i.e., before or more than 2.4 seconds after the heat stimulus), then juice was not delivered. Training in this task took several months, and once training was completed, the effects of morphine pretreatment were determined. Morphine produced antinociception insofar as it produced a dose-dependent decrease in thermal stimulus discrimination (i.e., after morphine treatment, monkeys failed more often to release the response key within 2.4 seconds of stimulus delivery, and therefore failed to earn juice delivery). Moreover, morphine effects were dependent on both stimulus 
intensity (the small heat-stimulus increase of $0.4^{\circ} \mathrm{C}$ was more sensitive to morphine antinociception than the higher stimulus increases of 0.6 and $1.0^{\circ} \mathrm{C}$ ) and stimulus modality (similar discrimination of a visual stimulus was not altered by morphine).

This approach to preclinical analgesic testing clearly presents many challenges, two of which are especially problematic. First, discrimination training is at best time-consuming, and in this case, took months to achieve before drug testing could commence. At worst, discrimination training might not be possible. For example, my laboratory has been unable to establish accurate discrimination performance of a visceral noxious stimulus (intraperitoneal acid) in rats using a two-lever discrimination procedure similar to procedures commonly used in studies of drug discrimination (Overton, 1988; Colpaert, 1999; Carter and Griffiths, 2009). Second, the requirement for extended training necessarily requires repeated exposure to the noxious stimulus, and this can complicate protocol approvals by regulatory agencies and alter nociceptive physiology and behavior by producing sensitization, inflammation, or neuropathy. These and other challenges have no doubt contributed to the dearth of preclinical research with noxious-stimulus discrimination.

Weighed against these two significant weaknesses are two potential strengths. First, preclinical paindiscrimination procedures can theoretically display high selectivity to distinguish between analgesics and drugs that produce nonselective behavioral disruption due to impaired motor function, motivation, or attention. In the procedure described above, for example, the analgesic morphine reduced the accuracy of noxiousheat discrimination without affecting trial initiation or the accuracy of visual discrimination (Thomas et al., 1992). A nonanalgesic negative control was not tested in these studies, but a nonanalgesic that produced deficits in motor function, motivation, or cognition (e.g., a $\kappa$ agonist) would have been expected to produce a nonselective decrease in rates of trial initiation for both noxious-heat and visual stimuli without necessarily altering the accuracy of responding in trials that were initiated.

A second strength of pain-discrimination procedures lies in their potential to model verbal pain reports in humans. In human pain assessment, the most commonly used endpoints are verbal behaviors guided by instruments such as the $0-10$ numeric rating scale or by questionnaires that ask respondents to endorse adjectives to describe their pain (Dworkin et al., 2005; Melzack and Katz, 2013). Most human verbal behavior is operant behavior occasioned by discriminative stimuli and maintained by consequent stimuli (Skinner, 1957). For example, in the case of the fast-food restaurant described above, the presence of a distinctive restaurant sign signals that verbal behavior involved in ordering a meal ("Cheeseburger, please") will produce delivery of that meal. Said another way, the restaurant sign as the $\mathrm{S}^{\mathrm{D}}$ "occasions" the expression of verbal ordering behavior $\mathrm{R}$ that is maintained by meal delivery as the $\mathrm{S}^{\mathrm{C}}$. Restaurant signs are "exteroceptive" stimuli that originate outside the body of the subject who emits the behavior, but operant verbal behavior can also be occasioned by "interoceptive" stimuli originating inside the subject. For example, food deprivation produces a constellation of interoceptive physiologic effects that we might learn to label verbally as "hunger," and the convergence of both exteroceptive stimuli (the restaurant sign) and interoceptive stimuli (physiologic effects of food deprivation) serves as a compound discriminative stimulus to signal not only that ordering a meal will produce a meal, but also that the meal is likely to be valued and consumed once received. This relationship is diagrammed in Fig. 7.

Noxious stimuli can also produce a constellation of interoceptive events that can serve as a discriminative stimulus for verbal behavior that may ultimately be reinforced. For example, verbally competent children or adults typically learn through a history of life experiences to use an increasingly sophisticated repertoire of words to describe the intensity, quality, and anatomic location of their internal pain experiences and to guide the delivery of treatment.

Figure 7 shows how pain-related verbal behavior might be occasioned by appropriate exteroceptive stimuli (e.g., a clinical context such as a doctor's office) and interoceptive discriminative stimuli (the physiologic and subjective effects produced by a painful injury) and reinforced by effective treatment delivery (e.g., analgesic administration). These verbal reports are the mainstay of human pain assessment, in part because of their ease of collection and their superficial appearance of precision given the presumption that the subject is both 1) in the best position to report on the dimensions of his or her own pain and 2) willing and able to deliver that report with accuracy. However, as with any operant behavior, the expression of verbal pain reporting depends not only on the discriminative stimuli that occasion it, but also on the consequent stimuli that follow it and the history of prior experience that has linked the experience of pain, the expression of verbal pain reports, and the delivery of consequent stimuli. In this regard, verbal pain reports might be unreliable as a pain measure for a variety of reasons. Three will be mentioned here. First, numeric pain-rating scales use the language of numbers $(1,2,3$, etc.), but these numeric labels provide only a qualitative ranking of pain states (i.e., they are ordinal variables rather than more quantitative interval or ratio variables), and they are anchored to a theoretical "worst pain imaginable" that can vary between subjects or within a subject over time (Kim, 2017). Thus, neither the increments between numeric ratings nor the range of possible ratings can be considered reliably stable within or between 
subjects. Second, operant conditioning may result in a failure to report pain despite the presence of pain. For example, an injured athlete who reports pain to a coach might lose the opportunity to compete, and such a consequent stimulus might reduce future verbal pain reports despite the presence of pain. Lastly, a reciprocal situation can also occur in which verbal pain reports occur despite the absence of pain. Of particular concern during the present crisis of opioid abuse, a verbal report of pain to an unethical physician might be sufficient to produce delivery of an opioid analgesic even if pain is absent, and that opioid delivery might reinforce future verbal pain reports in the absence of pain. Overall, verbal reporting as a type of pain-discrimination behavior is likely to remain a pillar of human pain assessment because the data are easy to collect and are often useful in guiding treatment; however, even in clinical research, concern over the reliability of verbal reporting has stimulated efforts to incorporate other, more objective metrics such as measures of pain-related functional impairment (Dworkin et al., 2005; Deyo et al., 2014). Pain-discrimination behavior is much more difficult to assess in laboratory animals.

3. Pain Stimulus as the Consequent Stimulus. A more common use of noxious stimuli in preclinical operant-conditioning procedures is as the consequent stimulus, and this relationship can be diagrammed as follows:

$$
\mathrm{S}^{\mathrm{D}} \rightarrow \mathrm{R} \rightarrow \mathrm{PS}
$$

In this role, the pain stimulus PS is delivered only if a particular discriminative stimulus is present and a particular response is emitted by the subject, and the
PS generally functions to punish responding (i.e., decrease the probability of the preceding response $\mathrm{R}$ ). Figure 8 shows one example of a preclinical procedure using a noxious stimulus as a consequent stimulus (Neubert et al., 2005, 2006; Anderson et al., 2013; Murphy et al., 2014). In this procedure, rats are placed into a testing cage (the features of which constitute the $\mathrm{S}^{\mathrm{D}}$ ) in which licking from a spout (the $\mathrm{R}$ ) results in delivery of sweetened condensed milk (the $S^{C}$ ) through the spout. To gain access to the spout, the subject has to press its face into an aperture lined with metal tubing that can be heated to controlled temperatures either by circulating water from an attached water pump or via a computer-controlled Peltier device. Thus, licking from the spout results not only in delivery of milk via the spout, but also in delivery of a thermal stimulus to the rat's face via contact with the heated surface (an additional $\mathrm{S}^{\mathrm{C}}$ ). Under these conditions, noxious thermal stimuli produce temperature-dependent decreases in the numbers of licking responses and milk deliveries. Thus, delivery of noxious thermal stimuli punishes licking behavior (i.e., decreasing the probability of licking). Moreover, thermal sensitivity in this procedure can be sensitized by treatment of facial skin with carrageenan to produce inflammation (Anderson et al., 2013) or capsaicin to produce neurogenic inflammation (Neubert et al., 2006), and in both cases, this thermal hypersensitivity to either a $45^{\circ} \mathrm{C}$ stimulus (Fig. 8) or a higher $48^{\circ} \mathrm{C}$ stimulus (unpublished data; J. Neubert, personal communication) can be reversed by a low dose of $0.5 \mathrm{mg} / \mathrm{kg}$ morphine (Neubert et al., 2005). Figure 8 also shows that the capsaicin-induced hypersensitivity to $48^{\circ} \mathrm{C}$ thermal punishment can be alleviated by the transient receptor potential V1 antagonist

A Verbal behavior occasioned by food-related stimuli

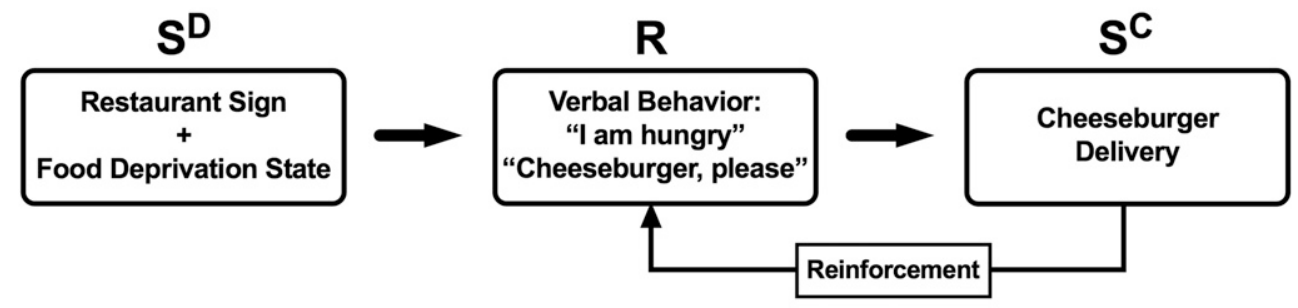

B Verbal behavior occasioned by pain-related stimuli

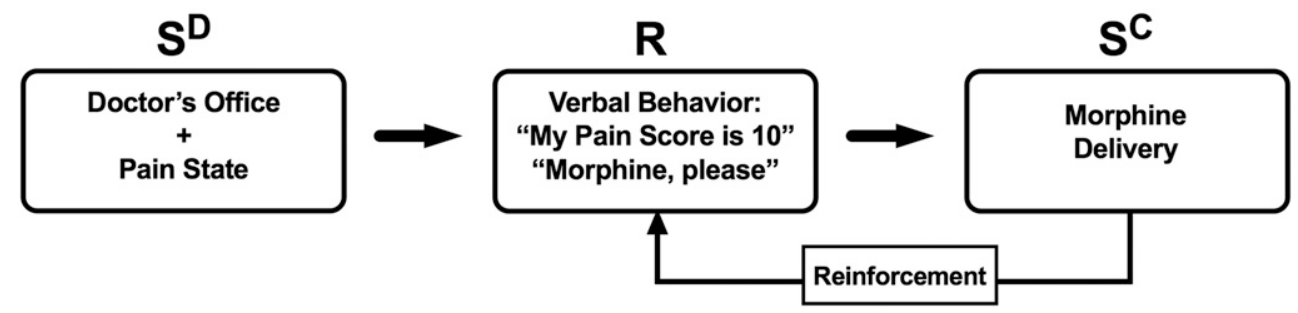

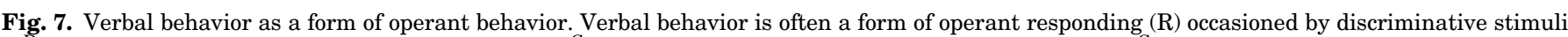

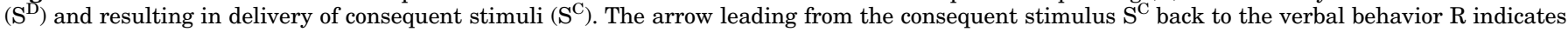
that the consequent stimulus increases the probability of (or "reinforces") the verbal behavior. 


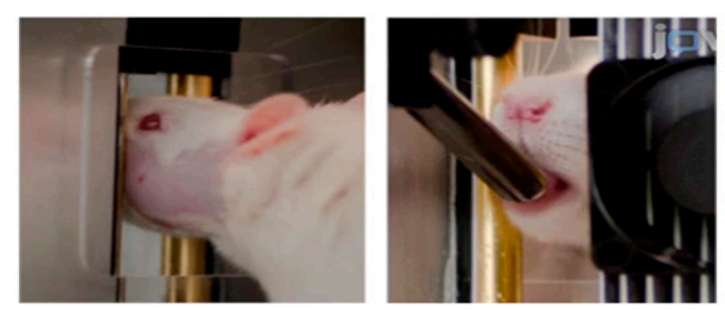

Licking Contact Events (Reward)

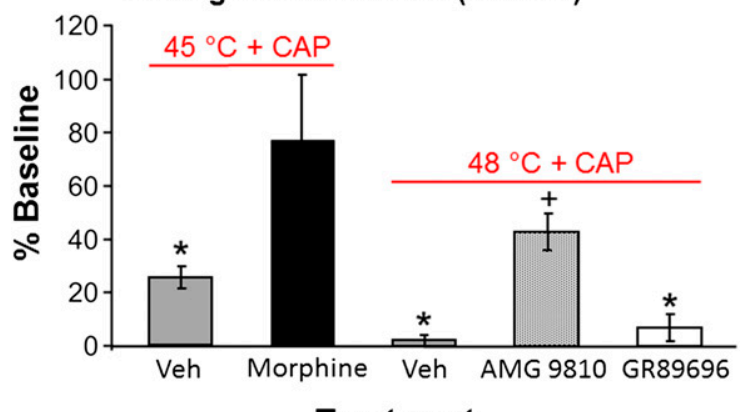

Treatment

Fig. 8. Noxious stimuli can serve in operant-conditioning procedures as consequent stimuli that punish behavior. The top two panels show photographs of a rat responding in an operant-based method called the Orofacial Pain Assessment Device, which can be used to assess thermal hypersensitivity. In this procedure, the subject must contact the stimulus surface (gold thermode) on either side of an aperture to access a drinking spout containing sweetened milk. The lower panel shows data for operant responding in rats tested in this procedure. Testing was completed for naive (untreated) animals at 45 or $48^{\circ} \mathrm{C}$, and rates of operant responding expressed as reward licking events were normalized as a percentage of baseline values at each respective temperature. Increasing temperatures decreased (i.e., punished) responding (data not shown; see Neubert et al., $2005)$, and treatment with capsaicin (CAP) $(0.1 \%$ topical cream) to the cheeks for 5 minutes produced hypersensitivity to thermal punishment, such that responding in rats treated with CAP and drug vehicle (Veh) was even lower after capsaicin treatment. Capsaicin-sensitized thermal punishment was alleviated by morphine $(0.5 \mathrm{mg} / \mathrm{kg})$ and by the transient receptor potential V1 antagonist AMG9810 $(30 \mathrm{mg} / \mathrm{kg} ;+, P<0.05 \mathrm{vs}$. Veh), but not by the $\kappa-2$ opioid receptor agonist GR89696 $(0.125 \mu \mathrm{g} / \mathrm{kg})$. $* P<0.05$ (significant pain-related punishment; i.e., fewer licking contact events than baseline); ${ }^{\#} P<0.05$ (antinociception; i.e., significantly more licking contact events after drug than after vehicle). This image was kindly contributed by Dr. John Neubert and adapted from Neubert et al. (2007).

(E)-3-(4-t-butylphenyl)-N-(2,3-dihydrobenzo[b] [1,4]dioxin6-yl)acrylamide (AMG9810) $(30 \mathrm{mg} / \mathrm{kg}$ ), but not by the $\kappa$ opioid receptor agonist methyl 4-[2-(3,4-dichlorophenyl) acetyl]-3-(pyrrolidin-1-ylmethyl)piperazine-1-carboxylate (GR89696) (Neubert et al., 2007). This procedure has also been adapted for use in mice (Neubert et al., 2008; Ramirez et al., 2015) and for use with noxious mechanical rather than thermal stimuli (Rohrs et al., 2015).

Other types of operant procedures have also been developed to establish thermal or mechanical punishment of operant behavior in rodents (Mauderli et al., 2000; Boada et al., 2016; Harte et al., 2016) and squirrel monkeys (Kangas and Bergman, 2014). These procedures include "place-escape/avoidance" procedures, in which subjects locomote within an environment with different zones, and entry into a subset of zones results in the delivery of a putative noxious stimulus (e.g., contact with a cooled or heated thermal plate or delivery of mechanical stimuli to the paw) (Pedersen and Blackburn-Munro, 2006; Fuchs and McNabb, 2012; Corder et al., 2019). These procedures take advantage of unconditioned exploratory behavior in rodents, and as a result, little or no training is required to establish baseline exploratory behavior between zones in the environment. Nonetheless, the procedures are considered here as operant punishment procedures because delivery of the noxious stimuli is contingent on the behavior of the subject (i.e., entry into zones where noxious stimuli are delivered), and delivery of noxious stimuli decreases that behavior. Pain is inferred from a decrease in time spent in the zones where noxious stimuli are delivered, and antinociception is expressed as an increase in this punished behavior.

There are two significant strengths to procedures that assess pain-related punishment of operant responding. First, operant learning with noxious stimuli plays a major role in shaping human behavior in both health and disease, and preclinical studies on expression and pharmacological modulation of these processes can contribute to translational research. In healthy subjects, noxious stimuli serve as robust teaching signals to punish maladaptive behaviors that might cause injury. For example, touching a red-hot stove burner will elicit an immediate withdrawal response as an unconditioned behavior, but that contact with noxious heat also serves as a consequent stimulus to punish future burnertouching behavior when the discriminative stimulus of a red burner is present. In injured or diseased subjects, hypersensitive pain states can also result in punishment of normally innocuous behaviors. For example, in patients with arthritis, normal movements such as walking can stimulate hypersensitive nociceptors in affected joints to punish further walking and produce a general profile of functional impairment (Grubb, 2004; Fearon et al., 2017). This type of hypersensitivity and punishment of behavior can again confer the adaptive advantage of reducing the probability of behaviors that could further damage the injured or diseased part of the body. However, pain-related punishment can also impede the performance of other adaptive behaviors like caring for children, meeting a job requirement, or escaping from danger. Moreover, movement-induced punishment of behavior can also impede participation in therapeutic physical therapy (Kanavaki et al., 2017).

A second advantage of punishment procedures is that noxious stimuli functioning as punishers decrease rates of the target behavior, and as a result, pain-related punishment can be conceptualized as a type of paindepressed behavior with many of the strengths described above for pain-depressed unconditioned behaviors (see section II.A.3). These strengths promote assay selectivity. In particular, effective analgesic drugs like morphine attenuate pain-related punishment to increase expression of the target behavior, whereas drugs that produce motor impairment 
do not produce false-positive analgesia-like effects (Neubert et al., 2005, 2007).

Punishment procedures are also associated with several disadvantages, including those mentioned above for pain-depressed unconditioned behaviors (see section II.A.3). Three other weaknesses specific to pain-related punishment of responding will be mentioned here. First, some degree of training is usually required to establish the baseline behavior to be punished, and that behavior must be established in an apparatus suitable for delivery of the noxious stimulus when the response $R$ is emitted. In a related point, punishment procedures work best when the punishing stimulus is delivered with rapid onset and short duration beginning immediately after the designated response, because punishing stimuli have their greatest impact on behaviors that immediately precede stimulus delivery (Pierce and Cheney, 2017). These temporal parameters are most easily achieved with thermal or mechanical stimuli, or some types of chemical stimuli (e.g., intravenous histamine delivery; Takada et al., 1992), that can be delivered with rapid onset and offset. Inflammatory, neuropathic, or disease-related pain stimuli thought to produce spontaneous pain with slower onsets and offsets are more difficult to incorporate directly into punishment procedures; however, hypersensitivity to punishing effects of evocative thermal or mechanical stimuli can be demonstrated in subjects with inflammation, neuropathy, or disease (e.g., Neubert et al., 2005; Pedersen and Blackburn-Munro, 2006; Fuchs and McNabb, 2012; Corder et al., 2019). Thus, punishment procedures can be used to assess the expression and pharmacological modulation of effects produced by relatively acute external pain stimuli, but they are not useful to assess chronic spontaneous pain.

A second relatively unexplored factor in pain-related punishment of behavior is the impact of the unconditioned or reinforcing consequent stimulus used to maintain behavior and permit detection of punishment. As discussed above, punishment procedures involve a compound consequent stimulus that includes both 1) a reinforcing or unconditioned stimulus sufficient to maintain stable rates of responding in the absence of the noxious stimulus, and 2) a noxious stimulus sufficient to punish baseline rates of responding maintained by the reinforcing/unconditioned stimulus. Previous studies have found that effectiveness of a given punisher depends not only on the intensity of the punisher but also on the effectiveness of the reinforcing stimulus (Azrin et al., 1963). Moreover, many current studies of pain-related punishment have used food as the positive reinforcing stimulus (Neubert et al., 2005; Kangas and Bergman, 2014), but other studies have used qualitatively different stimuli, such as escape from aversive light as a negative reinforcer (Mauderli et al., 2000; Harte et al., 2016) or a novel environment as an unconditioned stimulus. The impact of the intensity or type of reinforcing/unconditioned stimulus on pain-related punishment or drug-induced modulation of pain-related punishment has received little attention.

Lastly, an extensive body of literature has found that operant responding punished by aversive electric shock delivery is reversed by anxiolytic drugs (e.g., diazepam) but usually not by analgesic drugs (e.g., morphine) (Sepinwall et al., 1978; Patel and Migler, 1982; Pollard and Howard, 1990; Rowlett et al., 2006; Evenden et al., 2009). Although there are important differences between electric shock and more evolutionarily relevant modalities of thermal, mechanical, and chemical noxious stimuli (Corder et al., 2019), these results nonetheless suggest that anxiolytic drugs lacking analgesic effects, such as diazepam, have the potential to produce false-positive analgesic-like effects in procedures that use a noxious stimulus as the punisher. The sensitivity of pain-related punishment to reversal by diazepam or other nonanalgesic anxiolytics remains to be determined.

4. Pain Stimulus as a Contextual Stimulus. A final strategy for integrating a noxious "pain" stimulus into operant conditioning is in the role of a contextual stimulus as diagrammed here:

$$
\mathrm{PS} \rightarrow\left[\mathrm{S}^{\mathrm{D}} \rightarrow \mathrm{R} \rightarrow \mathrm{S}^{\mathrm{C}}\right]
$$

In this arrangement, operant responding (R) is occasioned by a nonpain discriminative stimulus $\left(\mathrm{S}^{\mathrm{D}}\right)$ and reinforced by a nonpain consequent stimulus $\left(\mathrm{S}^{\mathrm{C}}\right)$, and performance of this operant response is then evaluated in the context of a putative pain state produced by the pain stimulus (PS). One example of pain-related depression of operant behavior uses an intracranial self-stimulation (ICSS) procedure, in which rats implanted with microelectrodes in a brain reward area are initially trained to press a response lever for pulses of reinforcing electrical brain stimulation (Negus et al., 2010a; Negus, 2013; Negus and Miller, 2014). Figure 9 shows that increasing frequencies of electrical brain stimulation maintain increasing rates of operant responding and brain-stimulation delivery, and these data can be graphed as a "frequency-rate" curve. Once baseline responding is established, then intraperitoneal injection of dilute acid can serve as one type of noxious stimulus to produce behavioral depression, manifested as rightward and/or downward shifts in frequency-rate curves and decreases in the total number of stimulations delivered across all frequencies. Drugs can then be evaluated for their effectiveness to alleviate acid-induced ICSS depression. For example, Fig. 10 shows that acid-induced ICSS depression can be alleviated by morphine doses that do not significantly alter responding in the absence of the acid noxious stimulus (Altarifi et al., 2015), whereas the $\kappa$ 

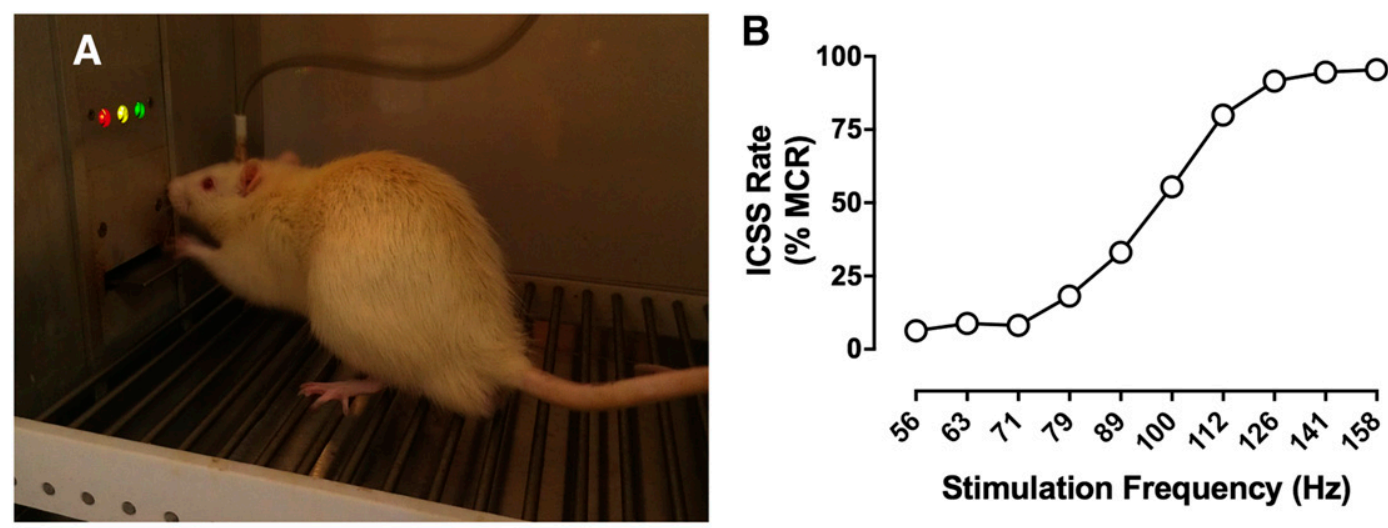

Stimulation Frequency $(\mathrm{Hz})$
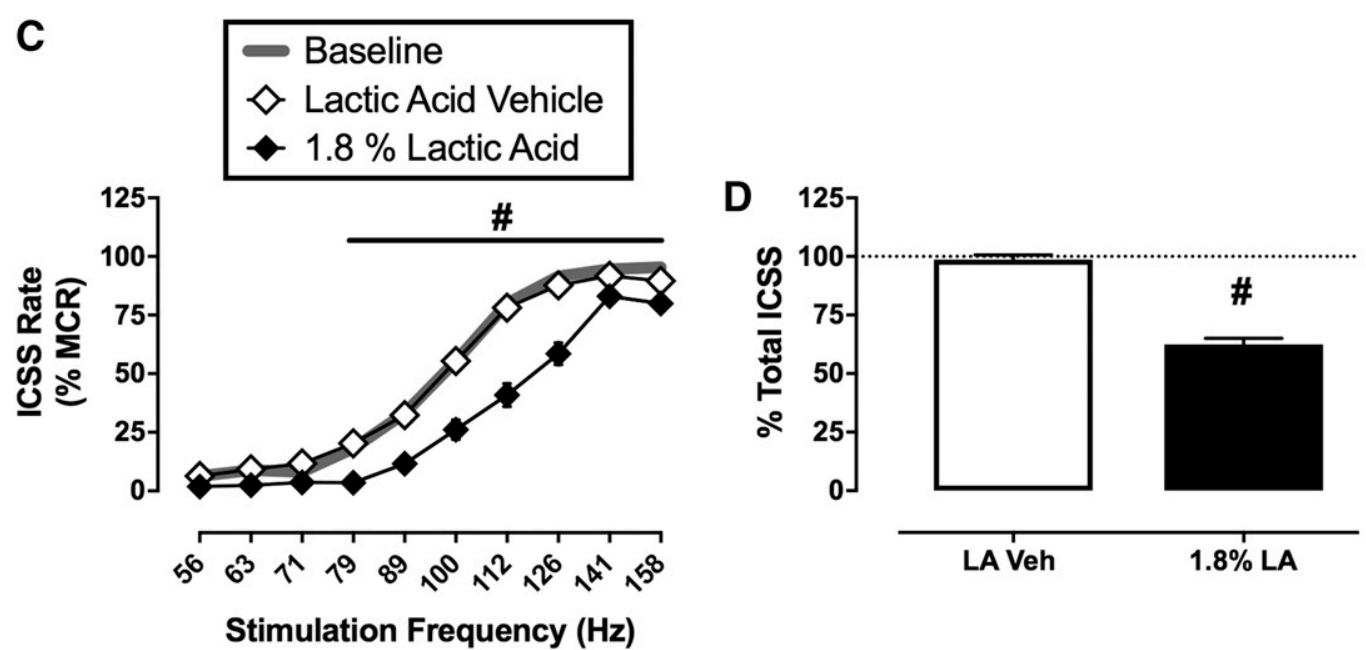

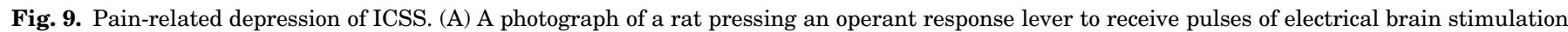

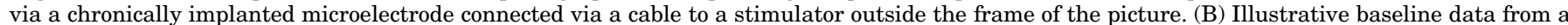

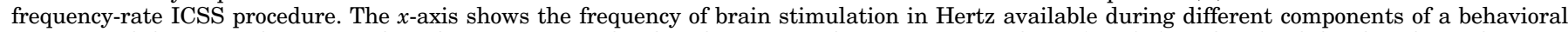

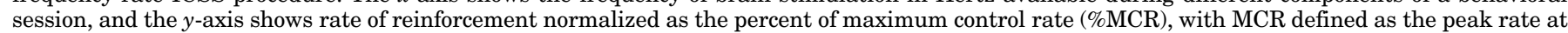

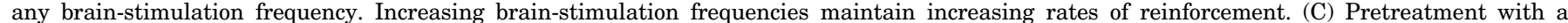

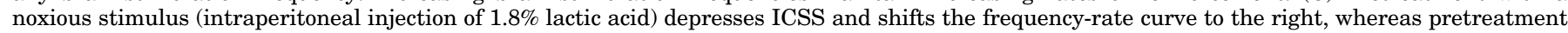

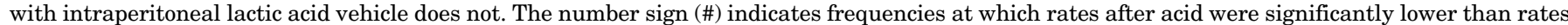

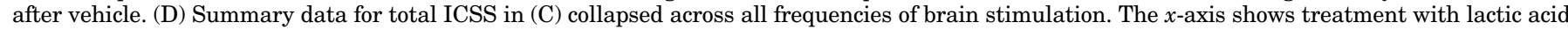

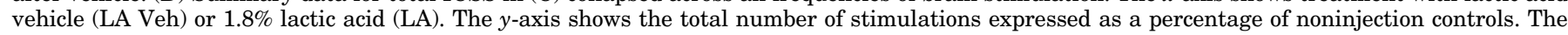
number sign (\#) indicates depression of ICSS by $1.8 \%$ lactic acid. Adapted from Negus (2013).

agonist nalfurafine was not effective (Lazenka et al., 2018).

In addition to improving assay selectivity for effective analgesic drugs, assays of pain-related depression of operant responding have two other strengths. First, baseline levels of operant responding can be maintained by different types of reinforcers (e.g., electrical brain stimulation, food, drug delivery), and reinforcer type can be evaluated as one determinant of pain-related behavioral depression. Second, the arrangement of discriminative stimuli, response requirements, and consequent stimuli in an operant behavioral procedure are defined by the "schedule of reinforcement," and schedule of reinforcement can be manipulated to probe motivational and cognitive processes and evaluate painand analgesia-related changes in those processes. Each of these two strengths will be briefly discussed in turn.

With regard to reinforcer type, food delivery is perhaps the easiest type of reinforcer to use in preclinical studies of operant responding, and several studies have reported pain-related and analgesic-reversible depression of food-maintained responding in rats (Martin et al., 2004 ; Ewan and Martin, 2014; Cone et al., 2018). The example in Figs. 9 and 10 described effects of an acute noxious stimulus (intraperitoneal acid injection) on responding maintained by electrical brain stimulation as a reinforcing stimulus. Although brain stimulation is not a natural reinforcer itself, it functions as a reinforcer by activating the mesolimbic dopamine brain-reward system, which consists of dopamine neurons that originate in the ventral tegmental area of the midbrain and project to forebrain regions that include the nucleus accumbens (Haber, 2014; Negus and Miller, 2014). The mesolimbic dopamine system is thought to mediate the reinforcing effects of both natural reinforcers and drugs of abuse and also to play a key role in setting mood states along a continuum from euphoria (high dopamine release in target regions such as the 

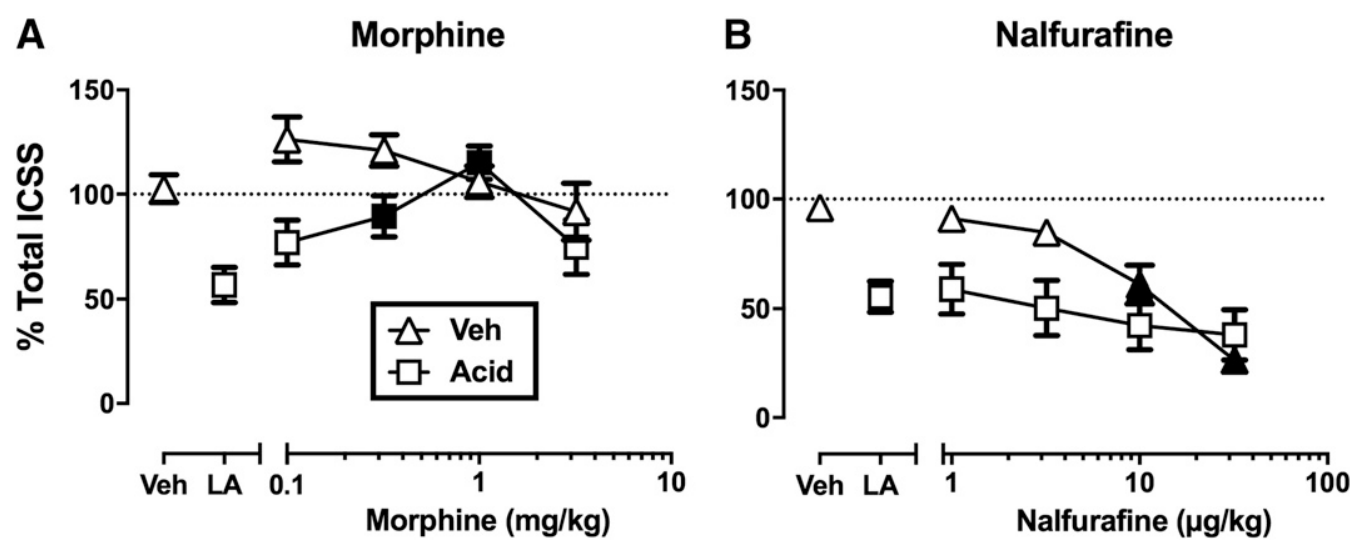

Fig. 10. The assay of pain-depressed ICSS can dissociate analgesics from nonanalgesics. (A and B) Effects of the $\mu$ opioid receptor agonist analgesic morphine (A) and the $\kappa$ opioid receptor agonist nonanalgesic nalfurafine (B) in rats treated with intraperitoneal lactic acid (LA) or with lactic acid vehicle (Veh). Abscissae show the dose in milligrams per kilogram. Ordinates show the percentage of total ICSS reinforcements collapsed across all brain-stimulation frequencies as shown in Fig. 9D. Treatment with lactic acid alone depressed ICSS, whereas vehicle treatment did not. Morphine blocked acid-induced ICSS depression in acid-treated rats at doses that did not affect ICSS in vehicle-treated rats. Conversely, nalfurafine failed to block acid-induced depression of ICSS up to doses that significantly decreased ICSS in vehicle-treated rats. Filled symbols indicate significant differences from LA in (A) or from Veh in (B) $(P<0.05)$. Data adapted from Altarifi et al. (2015) and Lazenka et al. (2018).

nucleus accumbens) to dysphoria (low dopamine release) (Leshner and Koob, 1999; Nestler and Carlezon, 2006; Baik, 2013). Consequently, ICSS procedures offer one strategy to investigate effects of pain stimuli on neural mechanisms of brain reward and mood. Indeed, intraperitoneal acid-induced depression of ICSS correlates with intraperitoneal acid-induced depression of mesolimbic dopamine release, and acid-induced depression of both endpoints can be selectively relieved not only by approved analgesics, but also by candidate analgesics that directly promote dopamine signaling (e.g., the antidepressant and dopamine uptake inhibitor bupropion) (Rosenberg et al., 2013; Leitl et al., 2014; Miller et al., 2015).

Drugs constitute another reinforcer type of particular interest in research with pain stimuli, because drug reinforcement in the context of a pain state may provide evidence for analgesic effects (Martin and Ewan, 2008). Although pain states decrease operant responding maintained by most reinforcer types, they may increase responding maintained by analgesic drug delivery, presumably because the analgesic relieves the aversive pain state (i.e., "negative reinforcement"). For example, early studies using oral consumption of drugs dissolved in drinking water found that an arthritis model in rats increased consumption of both the NSAID suprofen and the opioid fentanyl (Colpaert et al., 1980, 1982, 2001). The increased consumption of suprofen and fentanyl was interpreted as evidence for their increased reinforcing effects due to analgesia in the context of the inflammatory pain state. More recent studies have examined pain-stimulus effects on operant responding maintained by intravenous or intrathecal drug delivery. In one elegant example, rats could press an operant response lever to receive intrathecal injections of the $\alpha-2$ adrenergic agonist clonidine, which has analgesic effects in humans after intrathecal administration
(Martin et al., 2006). Rats with a spinal nerve ligation model of neuropathy self-administered intrathecal clonidine, whereas sham-lesioned rats did not, and the reinforcing effects of clonidine in the nerve-injured rats were attributed to clonidine's analgesic effects. Another study by this group examined opioid selfadministration in nerve-injured rats and found that nerve injury decreased self-administration of low doses of morphine and several other $\mu$ agonist analgesics; however, self-administration was retained for higher opioid doses that also alleviated mechanical hypersensitivity, and self-administration of these higher doses could be reduced by intrathecal clonidine in nerveinjured rats but not in sham rats (Martin et al., 2007). These results were interpreted to suggest that nerveinjury models of neuropathic pain decreased reinforcing effects of low opioid doses and rendered high-dose opioid reinforcement dependent on analgesic effects.

With regard to schedule of reinforcement, assays of operant behavior can be used to evaluate effects of pain stimuli and analgesic drugs on cognitive function (Boyette-Davis et al., 2008; Pais-Vieira et al., 2009; Freitas et al., 2015; Martin et al., 2017). Cognition embraces a range of processes that include attention, perception, working memory, long-term memory, executive function, language, and social cognition, and these different domains of cognitive function can be probed using operant procedures (Keeler and Robbins, 2011). For example, the relatively basic cognitive process of visual attention can be evaluated using a procedure called the "five-choice serial-reaction time task." In this task, rodents have access to five different nose-poke holes, and a nose-poke response in the correct hole is reinforced by delivery of a food pellet. The identity of the correct hole on any given trial is signaled by a brief light flash in that hole as the discriminative stimulus, and the location of the light flash is systematically varied 
during experimental sessions. Performance in this task, which depends in part on visual attention to the changing location of the light stimulus, could be impaired by intraperitoneal acid or by paw incision as acute pain stimuli, and these examples of pain-related impairment of performance could be blocked by NSAID and opioid analgesics (Martin et al., 2017; Ririe et al., 2018). Moreover, analgesics were not effective to block impaired attention due to a nonpain stimulus (treatment with the muscarinic acetylcholine receptor antagonist scopolamine).

The disadvantages of studies to examine effects of contextual pain stimuli on operant responding are similar to those described above for studies of paindepressed unconditioned behaviors (see section II.A.3). In particular, operant responding maintained either by electrical brain stimulation (Ewan and Martin, 2011, 2014; Legakis et al., 2018) or by food reinforcement (Ewan and Martin, 2014; Okun et al., 2016) is generally not depressed by either surgical or chemotherapy models of neuropathy despite the presence of mechanical hypersensitivity. These results complement studies of unconditioned behavior (see section II.A.3) in suggesting that preclinical models of putative neuropathic pain fail to produce a full spectrum of pain-related behaviors in laboratory animals and may not be adequate for research on treatments for dimensions of spontaneous pain that involve behavioral depression and functional impairment. An additional weakness is that, as with any operant procedure, some degree of training is required to generate stable behavioral baselines prior to the introduction of pain stimuli and candidate analgesic drugs. However, in contrast to studies of painpunished behavior (section II.C.3), the pain stimulus does not require rapid onset/offset temporal parameters. Rather, once the operant behavior has been trained, the pain stimulus can be delivered, and the onset, duration, and offset of any changes in behavior can then be tracked. Theoretically, operant responding has the potential to be sensitive to disruption by any persistent spontaneous pain that might be caused by putative chronic-pain stimuli such as surgical- or chemotherapy-induced neuropathy manipulations. However, as noted above, these putative chronic-pain stimuli generally have little effect on operant responding, suggesting that currently available chronic-pain models do not produce sufficient spontaneous pain to depress responding.

5. Summary. In summary, pain stimuli can serve as a discriminative stimulus, consequent stimulus, or contextual stimulus in operant behavioral procedures. Verbal pain reporting by humans is an operant behavior in which the pain state serves as an interoceptive discriminative stimulus, and pain relief by effective treatment is presumed to serve as one type of reinforcing consequent stimulus that maintains verbal pain reporting. Verbal pain reports are by far the most widely used behaviors in human pain assessment, and as a result, there is some value in seeking to model that behavior using discrimination procedures in laboratory animals. However, the wide use of verbal pain reports in human pain assessment is founded in part on the ease of data collection, and the training and testing of pain discrimination in laboratory animals is much more challenging. Moreover, verbal pain reporting in humans is vulnerable to inaccuracies that compromise both sensitivity and selectivity, and there are increasing efforts to supplement verbal reports with more objective measures of behavior in humans (Dworkin et al., 2005). In particular, behavioral depression and functional impairment are notable and objectively verifiable endpoints important in both the diagnosis and treatment of human pain. These endpoints can be assessed preclinically using procedures that employ pain stimuli as a consequent or contextual stimulus. Ultimately, the integrity of preclinical-to-clinical translational research might benefit more from efforts to investigate expression and treatment of these objective and clinically relevant behaviors, which can readily be studied in both humans and animals, rather than from efforts to develop pain-discrimination procedures in animals.

\section{Preclinical Antinociceptive Drug Profiles}

\section{A. The Antinociception Matrix}

The variety of "pain-stimulus" independent variables and "pain-behavior" dependent variables provide the foundation for an array of preclinical procedures for assessment of candidate analgesic drugs. Table 5 shows that this array can be visualized as a matrix, and results for a given drug (e.g., morphine in Table 5) can be displayed within this matrix to provide a preclinical antinociception drug (PAD) profile. It is the thesis of this review that $\mathrm{PAD}$ profiles will be more useful than results from any single procedure to guide translational research and prioritize candidate analgesics for advancement to clinical studies. Illustrative $\mathrm{PAD}$ profiles are provided below for selected drugs, and general recommendations are also provided below for sequencing preclinical studies to generate $\mathrm{PAD}$ profiles for other drugs. However, before proceeding to these topics, four caveats warrant mention.

First, the antinociception matrix provides a framework for consolidating and displaying data from a broad range of preclinical experiments. The matrix has two major dimensions, with rows for major classes of pain stimuli (as shown in Table 1; disease models excluded for simplification) and columns for major classes of pain behavior (as shown in Table 2 and discussed in detail in section II). Each cell within the matrix represents a family of procedures using the designated class of pain stimuli to generate the designated class of 
pain behaviors; however, further subcategories of different pain stimuli and pain behaviors clearly exist and may be relevant for certain applications. For example, the pain-stimulus category for neuropathy (bottom three rows in the matrix) as shown here consolidates results using both surgical and chemotherapy models, but there may be occasions when it is relevant to distinguish between these models. Similarly, the pain-behavior category for contextual painstimulus effects on operant behavior (far-right column, $\left.\mathrm{PS} \rightarrow\left[\mathrm{S}^{\mathrm{D}} \rightarrow \mathrm{R} \rightarrow \mathrm{S}^{\mathrm{C}}\right]\right)$ consolidates results using all schedules of reinforcement; however, different schedules of reinforcement can be used to assess different motivational and cognitive processes, and again, there may be occasions when it is relevant to distinguish between these models.

Second, the matrix also includes a third dimension using color to show drug effectiveness in the family of procedures represented by each cell. In the convention used here, green indicates that the drug is usually effective, red indicates that the drug is usually ineffective, blue indicates a pattern of mixed or ambiguous results, and white indicates that the drug has not been tested. References to support the color assignment are listed within the cell. This convention is intended to facilitate use of the matrix to compare drug effects across procedures, but with only three levels of effectiveness (effective, mixed, ineffective), the designated effect level has low resolution. Moreover, the quality or consistency of evidence leading to a summary of effectiveness may vary across studies. Future meta-analytic strategies could be developed to provide more quantitative and continuous summaries of drug effectiveness within each cell.

A third caveat to this matrix is that it does not include dimensions for other variables that may be of interest, such as the route of drug administration or the sex, strain, species, or age of the experimental subject. These types of variables could be incorporated by, for example, juxtaposing the PAD profiles for a given drug after systemic versus intrathecal administration or in male versus female subjects.

A final caveat is that procedures have yet to be developed for many cells in the matrix. Morphine is almost always used as a positive control during assay development because it is the prototype compound from a drug class ( $\mu$ opioid receptor agonists) that is well established to produce clinically effective analgesia across a wide range of pain states. Accordingly, blank cells in the PAD profile for morphine in Table 5 indicate that procedures for these cells have yet to be developed. In some cases, development of new procedures may not be feasible or warranted. For example, existing neuropathic pain stimuli do not produce reliable signs of spontaneous pain-stimulated unconditioned behaviors (e.g., grimacing; Langford et al., 2010), and as a result, there are no established models for drug effects on neuropathy-associated spontaneous unconditioned behaviors (row 9, column 1). Additionally, it was argued in section II.C.2 that development of preclinical "paindiscrimination" procedures may not be warranted even though operant discrimination behavior in the form of verbal reporting is the most commonly used outcome in assessment of human pain. In other cases, however, development of new procedures might be helpful. For example, there are currently no procedures to assess drug effectiveness to relieve inflammation + thermal stimulus-induced depression of unconditioned behavior (row 4, column 2). Such procedures might be useful to complement assays of inflammation-associated hypersensitivity of thermal stimulus-induced withdrawal reflexes. A possible and relatively simple procedure to

TABLE 5

Preclinical antinociception drug profile for morphine: a positive control

\begin{tabular}{|c|c|c|c|c|c|c|c|c|}
\hline & & \multicolumn{7}{|c|}{ Pain Behavior (Dependent Variable) ${ }^{a}$} \\
\hline \multicolumn{2}{|c|}{ Pain Stimulus (Independent Variable) } & \multicolumn{2}{|c|}{ Unconditioned Behavior US $\rightarrow$ UR } & \multicolumn{2}{|c|}{ Classical Conditioning CS+US; CS $\rightarrow$ UR } & \multicolumn{3}{|c|}{ Operant Conditioning $\mathrm{S}^{\mathrm{D}} \rightarrow \mathrm{R} \rightarrow \mathrm{S}^{\mathrm{C}}$} \\
\hline & & $\mathrm{PS} \rightarrow \mathrm{UR}$ & $\mathrm{PS} \rightarrow[\mathrm{US} \rightarrow \mathrm{UR}]$ & $\mathrm{CS}+\mathrm{PS} ; \mathrm{CS} \rightarrow \mathrm{UR}$ & $\mathrm{PS} \rightarrow[\mathrm{CS}+\mathrm{US} ; \mathrm{CS} \rightarrow \mathrm{UR}]$ & $\mathrm{PS} \rightarrow \mathrm{R} \rightarrow \mathrm{S}^{\mathrm{C}}$ & $\mathrm{S}^{\mathrm{D}} \rightarrow \mathrm{R} \rightarrow \mathrm{PS}$ & $\mathrm{PS} \rightarrow\left[\mathrm{S}^{\mathrm{D}} \rightarrow \mathrm{R} \rightarrow \mathrm{S}^{\mathrm{C}}\right]$ \\
\hline \multirow[t]{3}{*}{ Noxious } & Thermal & $1-4$ & & & & 10 & 11,12 & \\
\hline & Mechanical & 2,3 & & & & & 13 & \\
\hline & Chemical & $3-5$ & $6-9$ & Table 3 & & & & $4,14,15$ \\
\hline \multirow[t]{3}{*}{ Inflammation } & + Thermal & 16 & & & & & $24-26$ & \\
\hline & + Mechanical & $16-18$ & & Table 3 & & & 27 & \\
\hline & Spontaneous & 19,20 & $6,17,20-23$ & Table 3 & Table 4 & & & $21,28,29$ \\
\hline \multirow[t]{3}{*}{ Neuropathy } & + Thermal & 30,31 & & & & & & \\
\hline & + Mechanical & $30-32$ & & Table 3 & & & 13 & \\
\hline & Spontaneous & & 33 & & Table 4 & & & 34 \\
\hline
\end{tabular}

Citations are shown to illustrate effects produced by systemic administration of morphine in preclinical procedures using the designated pain stimulus to produce the

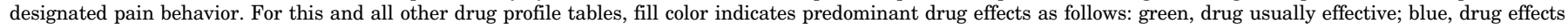
equivocal; and red, drug usually ineffective. No fill indicates drug not tested. Citations are not intended to be exhaustive, but rather to illustrate typical outcomes.

${ }^{a}$ Numbers correspond to the following references: 1) Morgan et al., 1999; 2) Craft et al., 2012; 3) Seguin et al., 1995; 4) Neelakantan et al., 2015; 5) Bagdas et al., 2016; 6)

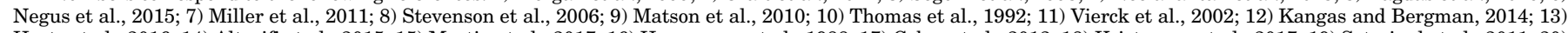

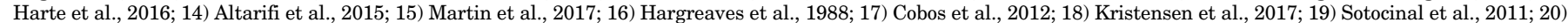

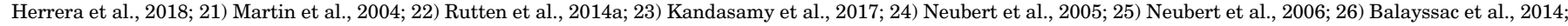

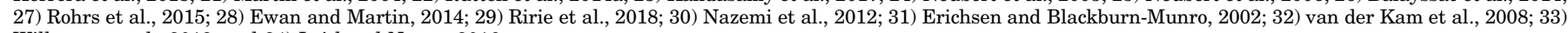
Wilkerson et al., 2018; and 34) Leitl and Negus, 2016. 
represent this cell could be assessment of food consumption on a hot plate heated to a threshold temperature (e.g., $\left.42^{\circ} \mathrm{C}\right)$ in rats or mice treated with intraplantar CFA. A predicted outcome is that thermal stimulation would reduce food consumption in CFA-treated subjects, and that morphine would dose-dependently block/reverse this effect. More generally, there is a research opportunity to develop new procedures for some cells in the matrix.

\section{B. $\mu$ Opioid Receptor Agonists: A Representative Positive Control}

With these caveats in mind, we can now turn to the $\mathrm{PAD}$ profile for morphine as a representative positive control. References cited within each cell in Table 5 include studies discussed above in section II, and most cells also include a few additional citations to illustrate both the types of procedures representative for a given cell and the effects of systemically administered morphine within those procedures.

As indicated by the predominance of green cells in Table 5, morphine is effective in most preclinical antinociception assays. This is not surprising given that morphine is a clinically effective analgesic often used as a positive control to validate new procedures, and procedures that fail to show effectiveness of morphine are generally considered to be of limited use for evaluating other candidate analgesics. The first column in the matrix $(\mathrm{PS} \rightarrow \mathrm{UR})$ shows morphine effects on pain-stimulated unconditioned behaviors that make up the majority of all preclinical research, and many more references could be cited to support effectiveness of morphine and other $\mu$ opioid receptor agonists in these procedures. Fewer studies have been conducted to assess effects of morphine and other $\mu$ agonists on other types of pain-related behaviors; however, morphine is also generally effective to alleviate pain-depressed unconditioned behaviors (column 2: PS $\rightarrow$ [US $\rightarrow \mathrm{UR}]$ ), pain-punished operant behavior (column 6: $\mathrm{S}^{\mathrm{D}} \rightarrow \mathrm{R} \rightarrow \mathrm{PS}$ ), and pain-depressed operant behavior (column $7: \mathrm{PS} \rightarrow\left[\mathrm{S}^{\mathrm{D}} \rightarrow \mathrm{R} \rightarrow \mathrm{S}^{\mathrm{C}}\right]$ ). Notably, morphine and other $\mu$ agonists typically have inverted-U shaped dose-effect curves in these procedures, with peak antinociception produced by relatively low doses, whereas higher doses produce sedation and motor impairment that obscure antinociception. Morphine also reliably alleviates pain-related conditioned place aversion (column 3: CS+US; CS $\rightarrow \mathrm{CR}$ ), and in the only published pain-discrimination study, it blocked the discriminative stimulus effects of noxious heat (column 5: $\mathrm{PS} \rightarrow \mathrm{R} \rightarrow \mathrm{S}^{\mathrm{C}}$ ).

There are two exceptions to the general antinociceptive effectiveness of morphine in Table 5 . In the first exception (row 2, column 6; reference 13), morphine was tested in an operant-escape apparatus consisting of an illuminated start box connected by a runway to a dark goal box (Harte et al., 2016). Features of the apparatus served as the discriminative stimulus $\mathrm{S}^{\mathrm{D}}$, locomotion down the runway to the goal box served as the response $R$, and escape from the presumably aversive light served as the reinforcing consequent stimulus $\mathrm{S}^{\mathrm{C}}$. Locomotion down the runway could be punished by elevation of probes in the runway floor to deliver a punctate mechanical stimulus to subjects' feet as an additional consequent stimulus $\mathrm{S}^{\mathrm{C}}$, such that probe elevation decreased latency to leave the start box. In rats with a chronic-constriction nerve-injury model of neuropathy, relatively low probe heights were sufficient to punish behavior, and morphine was effective to block this hypersensitivity to probe punishment, such that subjects left the start box more quickly after morphine treatment (row 8, column 6). The procedure has been further validated for evaluation of candidate analgesics in subjects with other types of inflammation- or neuropathy-induced mechanical hypersensitivity (Shepherd and Mohapatra, 2018). However, in control uninjured rats, higher probe elevations were required to punish behavior, and morphine failed to block punishment produced by these higher probe elevations (row 2, column 6). These results could be interpreted to suggest that either 1) punishment by higher probe elevations reflected a nonpain effect, such as physical obstruction of the runway path, that could not be attenuated by morphine, or 2) morphine failed to reduce sensitivity to the noxious stimulus produced by stepping on these higher probes, just as it also fails to block punishment by electric shock as discussed above (Sepinwall et al., 1978; Patel and Migler, 1982; Pollard and Howard, 1990; Rowlett et al., 2006; Evenden et al., 2009). Regardless of the interpretation, these results suggest limited utility of the procedure to detect mechanical antinociception in uninjured subjects.

The equivocal data from conditioned place-preference studies (column 4: PS $\rightarrow$ [CS+US; $C S \rightarrow C R]$ ) serve as an intriguing second exception to the general antinociceptive effectiveness of morphine. As summarized in Table 4, many early studies found that pain states blocked or attenuated morphine-induced place preferences, but these studies were not designed to test the hypothesis that morphine-induced pain relief would produce rewarding effects leading to a place preference. Rather, these studies were conducted to test the different hypothesis that pain states would attenuate opioid reward, and results supporting this hypothesis were deemed worthy of publishing by both authors and journals. The use of place conditioning to evaluate putative pain relief was not popularized until later, and these later studies hypothesized a different effect of pain states on morphine reward (i.e., that morphine analgesia in the context of a pain state would enhance the potency or effectiveness of morphine reward). Again, studies that supported the hypothesis were deemed worthy of publication by authors and journals. In both cases, the published articles supported the prevailing 
hypotheses, but the conflicting hypotheses seem to have generated conflicting results. It remains possible that these conflicting results reflect subtle differences in experimental parameters, but the fact that similar experimental designs have generated distinct results aligned more with their hypotheses than their methodology suggests the influence of publication bias (Andrews et al., 2016). At the very least, these results suggest caution in the use of place-preference procedures to examine putative rewarding effects of pain relief by candidate analgesics.

\section{C. к Opioid Receptor Agonists: A Representative Negative Control}

In the course of translational research on analgesic development, "false-positive" drugs will be identified that appear to have therapeutic potential based on preclinical findings but that ultimately fail to function as analgesics in humans. Apparent clinical failure can result not only from lack of efficacy but also from doselimiting safety concerns, species differences in the drug target, or poorly designed clinical trials, and characterization of a given drug as a false positive should be made with appropriate caution. Nonetheless, once identified and generally accepted by the field, false positives for a given pain indication can serve as valuable tools for use in refining preclinical research strategies. Specifically, false positives identify vulnerabilities in the existing research platform that allowed the drug to advance in the first place, and more importantly, they can be back-translated into preclinical research as part of an iterative process to develop new and more selective preclinical procedures (Fig. 1).

In this review, centrally acting $\kappa$ opioid receptor agonists have been used as a representative class of negative controls that has uniformly failed in the clinic due to inadequate effectiveness, unacceptable side effects, or both (Pande et al., 1996; Lazenka et al., 2018). Table 6 shows the PAD profile for a range of systemically administered $\kappa$ agonists in preclinical studies. Like morphine and other $\mu$ agonists, $\kappa$ agonists generally produce analgesic-like effects in conventional assays of pain-stimulated behaviors evoked by a wide range of different pain stimuli. These analgesic-like effects, coupled with evidence for low abuse liability, have been major factors in decisions to advance $\kappa$ agonists as candidate analgesics (Millan, 1990; Che et al., 2018). However, Table 6 also shows that $\kappa$ agonists, unlike morphine, have failed to produce analgesic-like effects in all other categories of pain behaviors for which they have been tested. These results provide one source of evidence to suggest that drug effects may vary across different pain behaviors even when those behaviors are produced by the same pain stimulus. These results further suggest that inclusion of procedures to evaluate these other pain behaviors can improve the selectivity and predictive validity of preclinical analgesic testing. Lastly, it is also clear from Table 6 that $\kappa$ agonists have been tested less thoroughly than morphine and other $\mu$ agonists in assays of pain-depressed unconditioned behavior, or in assays incorporating pain stimuli in Classical- or operant-conditioning procedures. These sparsely filled or empty cells provide opportunities for new research that might identify niche conditions under which $\kappa$ agonists might be effective.

The $\kappa$ agonist nalfurafine provides an especially instructive case study in failed analgesic drug development. Nalfurafine was initially developed as a candidate analgesic that produced antinociception in multiple assays of noxious, inflammatory, and neuropathic pain-stimulated behavior in mice, rats, and nonhuman primates (Nagase

TABLE 6

Preclinical antinociception drug profile for $\kappa$ opioid receptor agonists: a negative control

\begin{tabular}{|c|c|c|c|c|c|c|c|c|}
\hline & & \multicolumn{7}{|c|}{ Pain Behavior (Dependent Variable) ${ }^{a}$} \\
\hline \multicolumn{2}{|c|}{ Pain Stimulus (Independent Variable) } & \multicolumn{2}{|c|}{ Unconditioned Behavior US $\rightarrow$ UR } & \multicolumn{2}{|c|}{ Classical Conditioning $\mathrm{CS}+\mathrm{US} ; \mathrm{CS} \rightarrow \mathrm{UR}$} & \multicolumn{3}{|c|}{ Operant Conditioning $\mathrm{S}^{\mathrm{D}} \rightarrow \mathrm{R} \rightarrow \mathrm{S}^{\mathrm{C}}$} \\
\hline & & $\mathrm{PS} \rightarrow \mathrm{UR}$ & $\mathrm{PS} \rightarrow[\mathrm{US} \rightarrow \mathrm{UR}]$ & $\mathrm{CS}+\mathrm{PS} ; \mathrm{CS} \rightarrow \mathrm{UR}$ & $\mathrm{PS} \rightarrow[\mathrm{CS}+\mathrm{US} ; \mathrm{CS} \rightarrow \mathrm{UR}]$ & $\mathrm{PS} \rightarrow \mathrm{R} \rightarrow \mathrm{S}^{\mathrm{C}}$ & $\mathrm{S}^{\mathrm{D}} \rightarrow \mathrm{R} \rightarrow \mathrm{PS}$ & $\mathrm{PS} \rightarrow\left[\mathrm{S}^{\mathrm{D}} \rightarrow \mathrm{R} \rightarrow \mathrm{S}^{\mathrm{C}}\right]$ \\
\hline \multirow[t]{3}{*}{ Noxious } & Thermal & $1-4$ & & & & & & \\
\hline & Mechanical & $1-3,5$ & & & & & & \\
\hline & Chemical & $1-3,6,7$ & 8,9 & 6 & 10 & & & $7,11-13$ \\
\hline \multirow[t]{3}{*}{ Inflammation } & + Thermal & $8,9,14,15$ & & & & & 9 & \\
\hline & + Mechanical & 15,16 & & & & & & \\
\hline & Spontaneous & & 8 & & & & & \\
\hline \multirow[t]{3}{*}{ Neuropathy } & + Thermal & $17-20$ & & & & & & \\
\hline & + Mechanical & 19,20 & & & & & & \\
\hline & Spontaneous & & 20 & & & & & \\
\hline
\end{tabular}

Citations are shown to indicate effects produced by systemic administration of centrally acting and pharmacologically selective $\kappa$ opioid receptor agonists (U50,488,

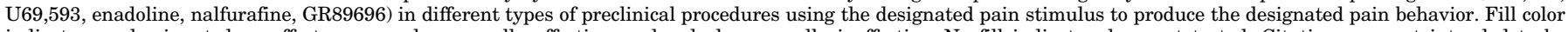

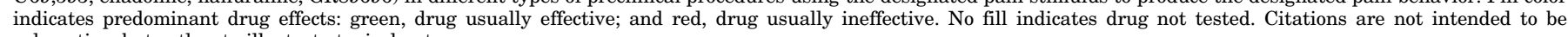
exhaustive, but rather to illustrate typical outcomes.

${ }^{a}$ Numbers correspond to the following references: 1) Seguin et al. 1995; 2) Endoh et al, 1999; 3) Hunter et al. 1990; 4) Barrett et al., 2002a; 5) Barrett et al., 2002b; 6)

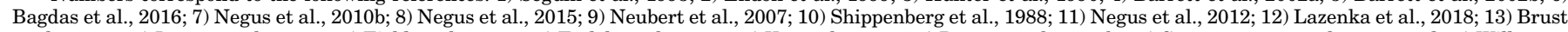

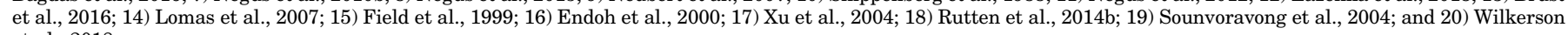
et al., 2018. 
et al., 1998; Endoh et al., 1999, 2000, 2001). Ultimately, nalfurafine was approved for clinical use in Japan, and it remains the only selective $\kappa$ agonist available for any clinical indication anywhere in the world; however, it was approved for the treatment of itch, not pain (Inui, 2015). Despite the promising preclinical results from conventional assays of pain-stimulated behaviors, no public results are available from any clinical trials with nalfurafine as a candidate analgesic, and there is no indication that nalfurafine is used off-label for treatment of pain. Thus, it appears that nalfurafine is similar to other centrally acting $\kappa$ agonists in being clinically untenable for pain treatment due to poor effectiveness and/or unacceptable side effects. In the only preclinical study consistent with its clinical failure, nalfurafine failed to produce a morphine-like blockade of intraperitoneal acid-induced ICSS depression in rats (an assay of pain-related depression of operant behavior, reference 12 in Table 6) (Lazenka et al., 2018).

Although centrally acting $\kappa$ agonists have not succeeded as candidate analgesics, $\kappa$ agonists with different pharmacodynamic or pharmacokinetic attributes continue to be developed, and novel approaches to preclinical drug assessment can play a role in evaluating new generations of $\kappa$ agonists. As one example, drugs can display pharmacodynamic bias in their efficacy and potency to activate different receptor-coupled signaling pathways, and $\kappa$ receptor ligands with bias to activate G-protein versus $\beta$-arrestin signaling pathways have emerged as one class of candidate analgesics that might retain $\kappa$ receptor-mediated antinociceptive effects while reducing undesirable side effects (Kenakin, 2015; Ranjan et al., 2017). In support of this possibility, one recent study found that the $\mathrm{G}$ protein-biased $\kappa$ agonist triazole 1.1 produced significant antinociception not only in conventional preclinical assays of pain-stimulated behavior but also in an assay of pain-related ICSS depression (Brust et al., 2016).

As another example, a class of $\kappa$ agonists is being developed with the goal of limiting pharmacokinetic distribution across the blood-brain barrier (Vanderah et al., 2008; Vadivelu et al., 2011; Hughes et al., 2013; Jones et al., 2016). After systemic administration, these compounds are restricted largely to the periphery, where they can act on peripheral $\kappa$ receptors, but they distribute poorly to the central nervous system and to $\kappa$ receptor populations in the brain and spinal cord. The working hypothesis with these compounds is that activation of peripheral $\kappa$ receptors, such as those located on primary nociceptor terminals, will be sufficient to produce clinically meaningful analgesia, and restricted distribution to brain will minimize centrally mediated side effects such as dysphoria and sedation. Peripherally selective $\kappa$ agonists produce antinociception in a broad range of preclinical procedures using pain-stimulated unconditioned behaviors; however, early generation peripherally selective $\kappa$ agonists failed to block intraperitoneal acid-induced depression of ICSS in rats (Negus et al., 2012). Although some compounds in this class have advanced to clinical trials, none have been approved for clinical use (Machelska et al., 1999; Arendt-Nielsen et al., 2009; Walker, 2018).

\section{Cannabinoid Receptor Agonists: A Representative Class of Candidate Analgesics}

Medical marijuana is one of the most prominent and controversial treatments to have emerged during the last 2 decades for the treatment of pain (National Academies of Sciences Engineering and Medicine, 2017; Romero-Sandoval et al., 2018; Vadivelu et al., 2018). U.S. federal law continues to classify marijuana as a Schedule I drug with no approved clinical indications, but marijuana has been legalized for recreational and/or medical use in more than 30 states and the District of Columbia. Moreover, despite weak evidence for clinical effectiveness as an analgesic (see below), pain is the most frequently endorsed reason for medical marijuana use in the United States and in other countries (Park and $\mathrm{Wu}, 2017$ ). The primary psychoactive constituent in marijuana is A9-tetrahydrocannabinol (THC), which acts as a low-efficacy agonist at cannabinoid 1 and cannabinoid 2 receptors, and many synthetic cannabinoid receptor agonists have also been developed as both experimental drugs and as candidate therapeutics for purposes that include pain management (Di Marzo and Petrocellis, 2006; Donvito et al., 2018). Table 7 shows the PAD profile for THC and several other pharmacologically similar drugs that function as agonists at cannabinoid 1 and cannabinoid 2 receptors (CP55940, WIN55212-2, nabilone) (for comprehensive reviews, see Martin and Lichtman, 1998; Donvito et al., 2018).

Three inferences can be drawn from this profile. First, THC and the other cannabinoid receptor agonists have been tested most extensively in assays of pain-stimulated unconditioned behaviors (column 1, PS $\rightarrow$ UR). Table 7 shows illustrative citations for each cell in this category, and many more studies could be cited to make the same points (Di Marzo and Petrocellis, 2006; Donvito et al., 2018). Overall, like both morphine (the positive control) and $\kappa$ opioid agonists (the negative control), THC and the other cannabinoid receptor agonists are reliably effective in all types of pain-stimulated unconditioned behaviors.

Second, THC and other cannabinoid receptor agonists have been tested only sparingly in procedures with other types of behavioral endpoints, and in most of those studies, cannabinoid agonists have failed to produce antinociception. Thus, these cannabinoids have failed to alleviate intraperitoneal acid-induced depression of wheel running in mice (Miller et al., 2012), intraperitoneal acid-induced depression of feeding and ICSS in rats (Kwilasz and Negus, 2012), 
noxious heat-induced punishment of food-maintained responding in squirrel monkeys (Kangas and Bergman, 2014), punishment of time spent in a place associated with mechanical paw stimulation in rats with chronic-constriction nerve injury (Pedersen and Blackburn-Munro, 2006), or formalin-induced depression of ICSS in rats (Leitl and Negus, 2016). In the two exceptions to this general finding, THC did alleviate depression of wheel running produced in female rats by application of allyl isothiocyanate to the dura (a model of migraine pain; Kandasamy et al., 2018), and CP55940 alleviated depression of marble burying produced in male mice by chronic-constriction injury to the sciatic nerve (Wilkerson et al., 2018). Cannabinoids have not yet been evaluated at all in assays of Classical conditioning with pain stimuli. There is significant opportunity for further research to examine effects of THC and other cannabinoids on pain-related behaviors other than conventional pain-stimulated unconditioned behaviors.

Lastly, it would appear that inclusion of these other behavioral endpoints could improve preclinical-to-clinical translation of results with cannabinoids. The uniformly robust effects of THC and other cannabinoid receptor agonists in all assays of pain-stimulated unconditioned behavior are clearly at odds with the weak, absent, or even hyperalgesic effects of cannabinoid agonists and cannabis products in humans evaluated for verbal pain reports elicited by acute exposure either to noxious experimental stimuli or to acute inflammatory stimuli such as postsurgical pain (Raft et al., 1977; Greenwald and Stitzer, 2000; Wallace et al., 2007; Kraft et al., 2008). Cannabinoids are generally thought to have their best therapeutic effectiveness for treatment of chronic pain, including neuropathic pain; however, even here, a growing body of evidence from clinical trials suggests that THC and cannabis products have weak and unreliable analgesic effectiveness despite being tested up to doses that produce problematic side effects (Finnerup et al., 2015; Aviram and Samuelly-Leichtag, 2017; Mücke et al., 2018; Stockings et al., 2018). Thus, results from clinical studies appear to align more closely to preclinical results from assays of pain-depressed and pain-punished behaviors than to results from assays of pain-stimulated unconditioned behaviors. More extensive testing of cannabinoids on a broader range of painrelated behaviors may help to clarify the circumstances under which cannabinoids are most likely to be effective.

\section{E. Recommendations for Preclinical Testing}

The antinociception matrix in Tables 5, 6, and 7 serves as a map of the preclinical experimental space within which pain-related behaviors are expressed and candidate analgesics are tested. Any given study will explore a subset of domains within the map, and over time, data for a given drug or drug class can accumulate to generate increasingly comprehensive topographies. Moreover, because the principles of unconditioned, classically conditioned, and operant conditioned behavior apply to humans as well as to laboratory animals, this same matrix could be used to organize and display results from human laboratory studies and clinical trials, and a comparison of PAD profiles for a given drug or drug class in animals and humans could serve as a tool to guide translational research. However, as with the exploration of a physical space, the progress of research through this experimental space must begin somewhere and pursue a path intended to reveal the most salient features of the terrain. At present, the most common path pursued by academic and industry laboratories is to work vertically down the first column of the antinociception matrix by evaluating drug effects on pain-stimulated unconditioned behaviors elicited by

TABLE 7

Preclinical antinociception drug profile for cannabinoid receptor agonists: a class of candidate analgesics

\begin{tabular}{|c|c|c|c|c|c|c|c|c|}
\hline & & \multicolumn{7}{|c|}{ Pain Behavior (Dependent Variable) ${ }^{a}$} \\
\hline \multicolumn{2}{|c|}{ Pain Stimulus (Independent Variable) } & \multicolumn{2}{|c|}{ Unconditioned Behavior US $\rightarrow$ UR } & \multicolumn{2}{|c|}{ Classical Conditioning $\mathrm{CS}+\mathrm{US}$; $\mathrm{CS} \rightarrow \mathrm{UR}$} & \multicolumn{3}{|c|}{ Operant Conditioning $\mathrm{S}^{\mathrm{D}} \rightarrow \mathrm{R} \rightarrow \mathrm{S}^{\mathrm{C}}$} \\
\hline & & $\mathrm{PS} \rightarrow \mathrm{UR}$ & $\mathrm{PS} \rightarrow[\mathrm{US} \rightarrow \mathrm{UR}]$ & $\mathrm{CS}+\mathrm{PS} ; \mathrm{CS} \rightarrow \mathrm{UR}$ & $\mathrm{PS} \rightarrow[\mathrm{CS}+\mathrm{US} ; \mathrm{CS} \rightarrow \mathrm{UR}]$ & $\mathrm{PS} \rightarrow \mathrm{R} \rightarrow \mathrm{S}^{\mathrm{C}}$ & $\mathrm{S}^{\mathrm{D}} \rightarrow \mathrm{R} \rightarrow \mathrm{PS}$ & $\mathrm{PS} \rightarrow\left[\mathrm{S}^{\mathrm{D}} \rightarrow \mathrm{R} \rightarrow \mathrm{S}^{\mathrm{C}}\right]$ \\
\hline \multirow[t]{3}{*}{ Noxious } & Thermal & $1-3$ & & & & & 8 & \\
\hline & Mechanical & $1,2,4$ & & & & & & \\
\hline & Chemical & $1,5,6$ & 6,7 & & & & & 6 \\
\hline \multirow[t]{3}{*}{ Inflammation } & + Thermal & $9-11$ & & & & & & \\
\hline & + Mechanical & $1,4,9$ & & & & & & \\
\hline & Spontaneous & & 12 & & & & & \\
\hline \multirow[t]{3}{*}{ Neuropathy } & + Thermal & $13-15$ & & & & & & \\
\hline & + Mechanical & $14-16$ & & & & & 18 & \\
\hline & Spontaneous & & 17 & & & & & 16 \\
\hline
\end{tabular}

Citations are shown to indicate effects produced by systemic administration of cannabinoid receptor agonists $[\Delta 9$-tetrahydrocannabinol, 2 -[(1R,2R,5R)-5-hydroxy-2-(3-

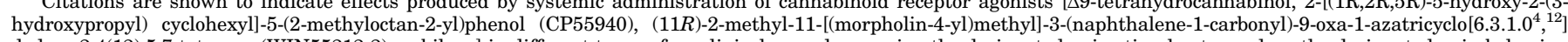

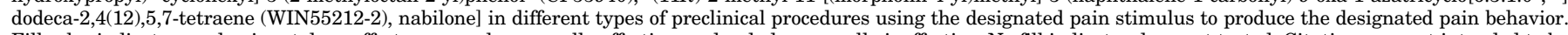

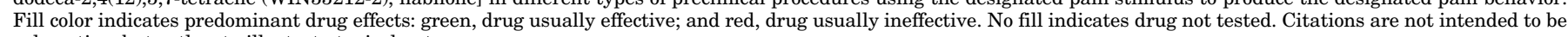
exhaustive, but rather to illustrate typical outcomes.

${ }^{a}$ Numbers correspond to the following references: 1) Sofia et al., 1975; 2) Tseng and Craft, 2001; 3) Fan et al., 1994; 4) Smith et al., 1998; 5) Moss and Johnson, 1980; 6)

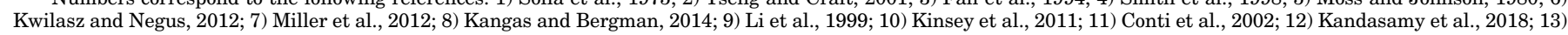
Mao et al., 2000; 14) Bridges et al., 2001; 15) Pascual et al., 2005; 16) Leitl and Negus, 2016; 17) Wilkerson et al., 2018; and 18) Pedersen and Blackburn-Munro, 2006. 


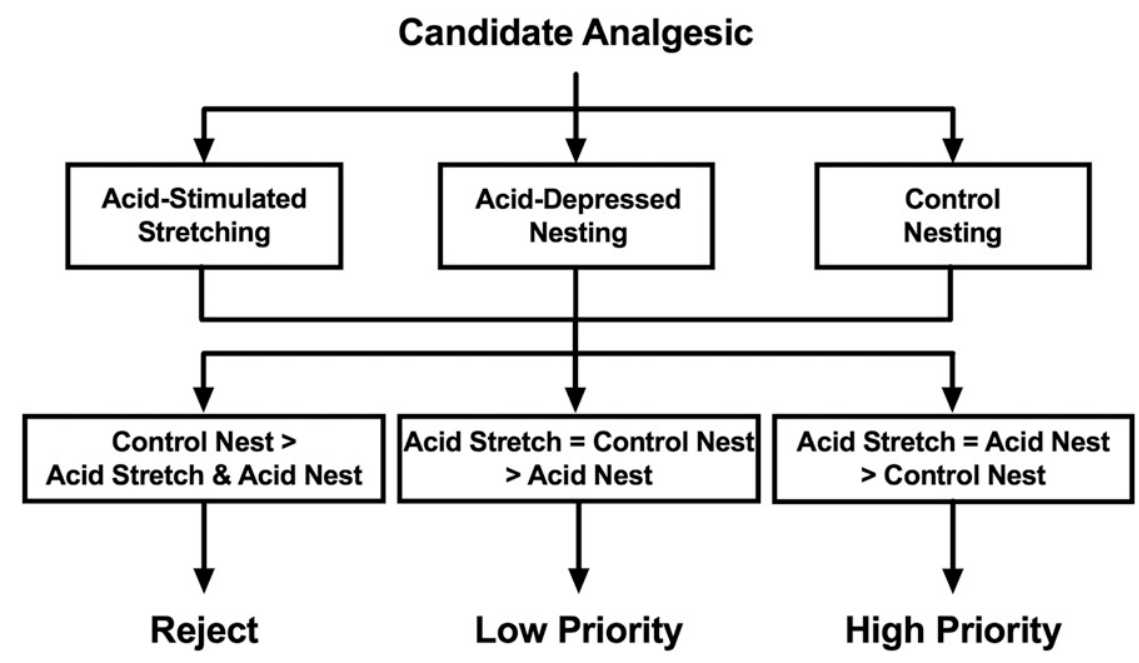

Fig. 11. Proposed path for initial testing with a candidate analgesic for acute inflammatory pain. One strategy for initial drug screening is to evaluate the drug potency, efficacy, and time course in mice using three assays: 1) intraperitoneal acid-stimulated stretching, 2) intraperitoneal acid-depressed nesting, and 3) control nesting in the absence of the noxious stimulus. Analgesia is indicated if a drug shows similar potency/effectiveness to block acidinduced stimulation of stretching and depression of nesting but has lower potency/effectiveness to impair control nesting (acid stretch $=$ acid nest $>$ control nest). This type of drug would be considered "high priority" for further testing. Conversely, analgesia is less likely if a drug shows similar potency/effectiveness to decrease acid-stimulated stretching and control nesting and has lower potency/effectiveness to block acid-induced depression of nesting (acid stretch $=$ control nest $>$ acid nest). Such a drug likely produces motor impairment with or without analgesia, and such a drug would be considered "low priority" for further testing. Lastly, analgesia is not indicated if a drug decreases control nesting but fails to produce antinociception in the assays of either acid-stimulated stretching or acid-induced depression of nesting (control nest $>$ acid stretch and acid nest). Such a drug could be rejected from further testing.

representative noxious, inflammatory, and neuropathic pain stimuli. This path has proved to be inefficient. It will now be argued that a better path is available that features a horizontal track of research across pain behaviors.

The prioritization of preclinical procedures into a path for initial drug testing should accommodate factors that include the physiologic relevance of the pain stimuli and pain behaviors to human pain states, the complementary strengths and weaknesses of the outcome measures with regard to assay sensitivity and selectivity, and the amenability of the path to high throughput. Figure 11 shows one path that might be useful in achieving these goals in analgesic development for treating acute inflammatory pain (e.g., postsurgical pain). This strategy relies on three procedures: 1) intraperitoneal acidstimulated stretching (a pain-stimulated unconditioned behavior), 2) intraperitoneal acid-depressed nesting (a pain-depressed unconditioned behavior), and 3) control nesting in the absence of the pain stimulus (a measure of nonselective drug effects on behavior).

Intraperitoneal acid is physiologically relevant as a pain stimulus insofar as inflamed tissue is often acidic relative to nondamaged tissue, and protons activate ion channels (e.g., transient receptor potential V1 and acid-sensing ion channels) expressed on primary nociceptors (Deval et al., 2010; Bray et al., 2013; Hung and Tan, 2018). As described above, intraperitoneal acidstimulated stretching and depression of nesting are useful as complementary outcome measures because of their complementary strengths and weaknesses. Intraperitoneal acid-stimulated stretching is notable for its sensitivity to the principal classes of clinically effective analgesics in the treatment of inflammatory pain ( $\mu$ opioid receptor agonists, NSAIDs), and this type of assay is also useful for rapid assessment of drug potency, efficacy, and time course to guide drug dosing and temporal parameters in other studies. However, as in all assays of pain-stimulated unconditioned behaviors, intraperitoneal acid-stimulated stretching can be reduced by nonselective behavioral effects such as sedation or motor impairment, and as a result, these assays lack selectivity to analgesic drugs and are extremely vulnerable to false-positive drug effects. Intraperitoneal acid-induced depression of nesting, by contrast, can compensate for this weakness by virtue of its selectivity. It is also sensitive to $\mu$ agonists and NSAIDs, but existing data suggest that drugs that produce nonselective behavioral disruption do not produce false-positive analgesic-like effects; rather, such drugs only exacerbate acid-induced depression of nesting. Moreover, assays of paindepressed unconditioned behaviors benefit from homology to pain-related behavioral depression and functional impairment as cardinal signs in human pain assessment. Taken together, these two procedures (intraperitoneal acid-induced stimulation of stretching and depression of nesting) constitute a horizontally organized set of cells in the antinociception matrix (row 3, columns 1 and 2). Evaluation of drug effects on these two pain behaviors can be further complemented by evaluation of drug effects 
on control nesting in the absence of the noxious stimulus to provide a direct measure of drug potency and effectiveness to disrupt general behavior as an undesirable side effect.

This trio of procedures is amenable to relatively high throughput. With regard to the independent variable, intraperitoneal acid is advantageous as a pain stimulus because it is easily quantified and delivered, produces a robust array of pain behaviors with a rapid onset and relative short duration of action ( $~ 1$ hour), and can be administered repeatedly to the same subject if needed for within-subject experimental designs or repeated dosing experiments. With regard to the dependent variables, stretching and nesting are unconditioned behaviors that require no training and can be quantitatively assessed as ratio variables amenable to parametric statistics. Moreover, these variables can be assessed with relatively little specialized equipment. Stretching requires only a clear chamber to permit observation, and although that observation can be performed in real time by an observer, it is best recorded by a video camera to minimize experimenter interactions with subjects during testing. These videos can then be scored later by blinded observers. Nesting requires only individually housed subjects and can be assessed in each subject's home cage using nesting material commonly provided to mice as part of standard husbandry practices. Photographs of the cage floor at the end of each session can again be scored by blinded observers.

Once drug effects in these three procedures are determined, they can then be compared to prioritize drugs for further testing. Analgesia is indicated if a drug blocks both acid-induced stimulation of stretching and depression of nesting with similar potencies, and has weaker potency or effectiveness to impair control nesting (acid stretching $=$ acid nesting $>$ control nesting). Such a drug could then be prioritized for further testing to determine mechanisms of action, effects of chronic treatment, or effects in other procedures using other pain stimuli or pain behaviors. Conversely, analgesia is less likely if a drug decreases both acid-stimulated stretching and control nesting with similar potencies but fails to block acid-induced depression of nesting (acid stretching = control nesting $>$ acid nesting). This type of profile could be produced by a drug that produces both analgesic effects and motor impairment at similar doses, but this profile could also reflect drug-induced motor impairment without analgesia. These possibilities could be dissociated using follow-up approaches (e.g., place-conditioning procedures); however, further testing with such a drug would likely have lower priority, because even if the drug did have analgesic effects, motor impairment might be expected as a dose-limiting side effect that could impede delivery of analgesic doses. Lastly, a drug that was ineffective in assays of both acid-stimulated stretching and acid-induced depression of nesting up to doses that impaired control nesting would have the lowest potential as an analgesic and the lowest priority for further testing (control nesting $>$ acid stretching and acid nesting).

It should be emphasized that this is only one of many possible strategies that could be developed for more efficient use of preclinical procedures for assessment of candidate analgesics. The approach described above focuses on the use of an acute noxious stimulus to model pain states associated with the tissue-acidosis component of acute injury and inflammation, and drugs identified with this approach might serve as useful alternatives to $\mu$ opioid agonists and NSAIDs for treatment of acute inflammatory pain. This is not trivial. For example, the National Institutes of Health recently announced a major research initiative that seeks to enhance pain management by discovering new nonaddictive analgesics that could replace highly addictive opioid analgesics (https://www.nih.gov/researchtraining/medical-research-initiatives/heal-initiative). Although opioids are used to treat a wide range of pain conditions, one major use is for relatively shortterm pain states related to injury and inflammation, such as postsurgical pain. As a result, the discovery of new analgesics to treat short-term pain states could enable a sizeable reduction in prescription opioid use.

Nonetheless, analgesic discovery for more chronic inflammation-, neuropathy-, or disease-related pain states is also critical, and analgesic development for these chronic pain states would also benefit from improved preclinical research strategies that capitalize on a horizontal path through the matrix. The most widely used endpoints in preclinical research on chronic pain are measures of hypersensitive withdrawal responses from mechanical or thermal stimuli. Procedures that rely on these pain-stimulated unconditioned behaviors generally have the strengths and weaknesses of other "sensitive" preclinical procedures (see section II.A.2), but these procedures can be complemented by potentially more selective procedures that assess hypersensitivity to behavioral depression or punishment by these same mechanical and thermal stimuli (sections II.A.3, II.C.3, and II.C.4). For example, several studies have compared treatment effects on hypersensitive paw withdrawal (a painstimulated behavior) and place escape/avoidance (a pain-punished behavior) elicited by acute mechanical or thermal stimuli in subjects with surgical neuropathy manipulations (LaBuda and Fuchs, 2000; Pedersen and Blackburn-Munro, 2006; Corder et al., 2019). Morphine generally reduces both types of pain behavior, and intriguingly, some treatments relieve pain-punished behaviors without affecting hypersensitive withdrawal responses (e.g., duloxetine; Pedersen 
and Blackburn-Munro, 2006). Although such findings could reflect anxiolysis or learning impairment (see section II.C.3), they are often interpreted to suggest that the treatment under consideration blocks "affective" versus "sensory" dimensions of pain; however, the translational implications of such findings remain to be determined. For example, it is not yet clear whether drugs that relieve pain-punished behaviors without relieving hypersensitive withdrawal responses should be prioritized for advancement to clinical testing.

The sequential use of procedures that assess drug effects on hypersensitive pain-stimulated and painpunished behaviors is also associated with two other significant challenges. First, this approach relies on measures of hypersensitive responses to acutely applied external stimuli, but clinically relevant chronic pain in humans more often manifests as spontaneous pain that is independent of external stimuli and sufficient to impair function and quality of life (Backonja and Stacey, 2004; Dworkin et al., 2005). Different approaches will be required for preclinical assessment of the expression and treatment of chronic spontaneous pain, but as reviewed above, current experimental manipulations for producing putative chronic pain in rodents often produce little or no evidence of impaired function as sign of spontaneous pain. As a result, these procedures provide little opportunity either to validate the existence of chronic spontaneous pain or to investigate effects of candidate analgesics on functional impairment that might be caused by chronic spontaneous pain. No doubt, there will be continued efforts to develop methods for sensitive detection of chronic pain-associated functional impairment. At present, conditioned place-preference procedures appear to lay the strongest claim to usefulness for assessment of chronic spontaneous pain, and these CPP procedures have the strengths and weaknesses described above in section II.B.3.

A second challenge is the lack of robust positive controls or consensus negative controls that can be used to validate preclinical procedures for chronic pain. For example, gabapentin is often used clinically to treat various types of neuropathic pain, but prevailing clinical data suggest that it has limited clinical effectiveness both in absolute terms and in comparison with other analgesics such $\mu$ opioid receptor agonists or monoamine uptake inhibitor antidepressants (Pachman et al., 2011; Finnerup et al., 2015; Wiffen et al., 2017). Despite its marginal clinical effectiveness, gabapentin is often used to validate sensitivity of preclinical assays of neuropathic pain, and it often produces antinociception indistinguishable in magnitude either from effects of other positive controls known to be more effective clinically or from the maximum possible effect attainable in the procedure (Park et al., 2013; Simmons et al., 2014; Wilkerson et al., 2018). Presumably, one goal of analgesic development for neuropathic pain is to improve on existing but imperfect compounds like gabapentin; but to accomplish this task, it will be necessary to use procedures capable of detecting such improvement. Despite these caveats, the general recommendation applies that analgesic testing for any type of pain state will benefit from use of multiple pain behaviors that have complementary strengths and weaknesses. Moreover, as drugs advance to clinical testing, both effective and ineffective drugs can be back-translated as positive and negative controls to refine the preclinical testing strategy and improve both sensitivity and selectivity in analgesic drug discovery (Fig. 1).

\section{Other Considerations}

\section{A. Analgesic Tolerance}

Analgesics are often administered repeatedly for periods of days, months, or even years to treat sustained pain states, and analgesic tolerance is a potential barrier to effective pain management (Jamison and Mao, 2015). The expression and mechanisms of tolerance, and especially opioid tolerance, have been extensively studied in preclinical research by evaluating changes in drug-induced antinociception during regimens of repeated drug treatment (Colpaert et al., 2001; Vanderah et al., 2001; Zuo, 2005; Akbarali and Dewey, 2017). Tolerance to the antinociceptive effects of $\mu$ opioid agonists like morphine is a robust preclinical phenomenon, many different mechanisms of opioid antinociceptive tolerance have been identified, and this mechanistic research has led to proposals of many different strategies to reduce opioid analgesic tolerance in humans. However, this preclinical research has relied almost exclusively on assays of painstimulated unconditioned behaviors, and none of this research has led to approved treatments to mitigate opioid analgesic tolerance in clinical settings. Consequently, research on opioid antinociceptive/analgesic tolerance represents another area of largely failed preclinical-to-clinical translation.

The narrow preclinical focus on pain-stimulated unconditioned behaviors may contribute to this poor translation of results. This review has emphasized the point that preclinical pain stimuli produce a range of different pain behaviors that can be differentially modified by acute drug treatments, and this principle also applies to the potential for differential expression and mechanisms of antinociceptive tolerance to repeated drug treatment. For example, one study compared the development of antinociceptive tolerance produced by a regimen of repeated morphine treatment in rats tested for a pain-stimulated unconditioned behavior (intraperitoneal acid-induced stimulation of stretching) and a pain-depressed operant behavior (intraperitoneal acid-induced depression of ICSS) (Altarifi and Negus, 2015). Complete tolerance developed to morphine antinociception in the assay of intraperitoneal acid-stimulated stretching, but 
morphine remained effective to alleviate intraperitoneal acid-induced depression of ICSS. Thus, morphine antinociception in the assay of pain-depressed operant behavior was resistant to tolerance. This agrees with the clinical observation that many patients with chronic pain can be maintained on stable opioid doses for long periods, and that dose escalation when it occurs often reflects disease progression and increased pain rather that decreased opioid analgesic effectiveness due to pharmacodynamic tolerance (Foley, 1995). Indeed, tolerance to opioid-induced side effects like sedation and nausea/emesis often facilitates the use of opioids for pain treatment (Chapman et al., 2010). Overall there is a clear opportunity for research on the expression and mechanisms of opioid antinociceptive tolerance in assays using outcome measures other than pain-stimulated unconditioned behaviors. Moreover, Classical- and operantconditioning factors that influence pain expression can also influence tolerance to analgesic effects of drugs (Siegel, 1978), and the interaction between pain states, drug tolerance, and conditioning is largely unexplored.

\section{B. Safety}

An important consideration in any analgesic development program is the discovery of drugs that are safe as well as effective. Safety assessments rely on a multitude of outcome measures concerned with drug effects on multiple physiologic and behavioral endpoints; however, novel behavioral measures that can be used to assess antinociception can also be used to provide one source of useful information on drug safety. Assays of pain-depressed unconditioned behavior, painpunished operant behavior, and pain-depressed operant behavior all rely on conditions that support high and stable rates of the baseline behavior. To assess antinociception, these high baseline rates of behavior are depressed or punished by a pain stimulus, and candidate analgesics are evaluated for their effectiveness to block effects of the pain stimulus and restore behavioral rates to baseline levels. A measure of drug safety can be determined in these same procedures by evaluating drug effects on the same behavior in the absence of the pain stimulus. Virtually all drugs will impair behavior at high enough doses due to undesirable effects that could include sedation, convulsions, or paralysis. Thus, these procedures provide the framework for directly comparing drug potency and effectiveness to produce antinociception (drug effects on the target behavior in the presence of the pain stimulus) with potency and effectiveness to produce a general measure of behavioral toxicity (drug effects on the same behavior in the absence of the pain stimulus). Highpriority drugs for further consideration would be those that have higher potency to alleviate pain-related depression of the target behavior than to produce behavioral toxicity as indicated by impairment of that behavior in the absence of the pain stimulus. For example, in the nesting procedure in mice shown in Fig. 2, morphine was 7.5-fold more potent to block intraperitoneal acid-induced depression of nesting $\left(\mathrm{ED}_{50}=0.72 \mathrm{mg} / \mathrm{kg}\right)$ than to decrease control nesting in the absence of the acid noxious stimulus $\left(\mathrm{ED}_{50}=\right.$ $5.37 \mathrm{mg} / \mathrm{kg}$ ) (Negus et al., 2015). This type of ratio can be calculated and compared across drugs to provide one measure of drug safety.

Place-conditioning, ICSS, and drug self-administration procedures can also be used to evaluate abuse potential as one type of undesirable effect especially relevant to the quest for alternatives to opioid analgesics. In placeconditioning procedures, abuse potential is indicated if drug administration in the absence of a pain stimulus produces a conditioned place preference (Tzschentke, 2007). In ICSS procedures, abuse potential is indicated if drug administration in the absence of a pain stimulus increases ICSS rates (Negus and Miller, 2014). In drug self-administration, abuse potential is indicated if some dose of drug maintains higher rates of self-administration than drug vehicle (Carter and Griffiths, 2009; O'Connor et al., 2011). Thus, in all three types of procedure, drug effects in the presence of a pain stimulus can provide evidence of antinociception, whereas drug effects in the absence of the pain stimulus can provide evidence of abuse potential.

\section{Mechanisms of Pain and Analgesia}

Procedures used to evaluate effects of candidate analgesics are also used to investigate underlying mechanisms of pain behaviors and identify new receptor targets for drug development. Research studies with novel pain behaviors described in this review are already shedding new light on mechanisms that may contribute to pain in humans and serve as druggable targets in analgesic drug development. For example, studies using ICSS and place-conditioning procedures have converged in highlighting a role for the mesolimbic dopamine system in the expression and treatment of pain. As noted in section II.C.4, the mesolimbic dopamine system consists of dopaminergic neurons that project from the ventral tegmental area to the nucleus accumbens, and this system plays a key role in mood and motivated behavior (Mogenson et al., 1980; Nestler and Carlezon, 2006; Ikemoto, 2010). Operant ICSS behavior can be maintained by electrical stimulation of excitatory inputs to the mesolimbic dopamine system, and as a result, the finding that pain stimuli such as intraperitoneal acid can depress ICSS suggested that pain stimuli might also depress activity of the mesolimbic dopamine system (Pereira Do Carmo et al., 2009). Subsequent studies used in vivo microdialysis to confirm that intraperitoneal acid reduced dopamine release in the nucleus accumbens, and that analgesic drugs selectively blocked intraperitoneal acid-induced depression of both behavior and nucleus accumbens dopamine 
release (Leitl et al., 2014; Miller et al., 2015). This evidence to suggest that pain states can depress mesolimbic dopamine activity is complemented by additional evidence to suggest that pain relief reciprocally stimulates mesolimbic dopamine activity (Xie et al., 2014; Watanabe and Narita, 2018). For example, in mice treated with either a paw-incision model of postsurgical inflammatory pain or a spinal nerve ligation model of neuropathic pain, commonly used clinical analgesics were effective to produce both a conditioned place preference and an increase in nucleus accumbens dopamine release without altering either of these measures in the absence of the pain stimuli (Xie et al., 2014). These preclinical findings agree with clinical evidence for a role of dopamine dysfunction in pain (Borsook et al., 2007; Wood, 2008; Martikainen et al., 2018). Additionally, an implication of these findings is that existing or candidate antidepressant drugs such as bupropion or amitifadine, which block dopamine as well as serotonin and/or norepinephrine transporters to increase synaptic levels of these neurotransmitters, might warrant further consideration as treatments for the depressant effects of pain (Semenchuk et al., 2001; Rosenberg et al., 2013; Leitl et al., 2014; Miller et al., 2015; Ashrafi et al., 2018).

\section{Conclusions}

A consistent theme throughout this review has been the emphasis on strategies to improve preclinical-toclinical translation of research with candidate analgesic drugs. In conclusion, four specific recommendations are offered to support this goal. First, translational research for analgesic drug development, as for any type of drug development, will benefit from alignment of outcome measures in animals and humans (Yu, 2011). This review has identified a taxonomy of pain-related unconditioned, classically conditioned, and operant conditioned behaviors that applies to both animals and humans, and translational fidelity is likely to be greatest when using a preclinical procedure to predict drug effects on that same type of behavior in humans (Negus, 2018). For example, if one is interested in predicting potential for a candidate analgesic to relieve pain-related behavioral depression and functional impairment in humans (as recommended by the Initiative on Methods, Measurement, and Pain Assessment in Clinical Trials; Dworkin et al., 2005), then translational research would be best served by preclinical studies to examine effects of that drug on pain-related depression/ punishment of unconditioned or operant behavior. Unfortunately, preclinical and clinical research are now badly misaligned. Specifically, the most widely used endpoints in preclinical studies measure withdrawal responses of one sort or another (i.e., pain-stimulated unconditioned behaviors), whereas the most widely used endpoints in human studies use verbal reports (i.e., a type of operant behavior in which the pain stimulus serves as a discriminative stimulus). This misalignment of behavioral endpoints has likely contributed to the poor record of preclinical-to-clinical translation of results with many candidate analgesics.

Second, this review has described the "antinociception matrix" as a tool to map results from preclinical drug testing onto an experimental space with coordinates defined by the range of possible pain stimuli and pain behaviors. Additionally, a novel path has been proposed for initial data collection within this experimental space. Current practices emphasize a "vertical" path that varies the pain stimulus while relying on a single category of pain behavior (pain-stimulated unconditioned behaviors). Pursuit of this path has been strikingly inefficient in generating effective analgesics for use in humans, and instead, it has generated a high number of false positives that appear promising based on preclinical results but then fail in clinical studies. Centrally acting $\kappa$ opioid receptor agonists exemplify this type of failed translation. The alternative path recommended here emphasizes a "horizontal" path that evaluates multiple pain behaviors produced by a pain stimulus of interest. This path is proposed to be advantageous because it includes pain behaviors (e.g., pain-depressed behaviors) of particular clinical relevance, and because procedures that use different pain behaviors (e.g., pain-stimulated and pain-depressed unconditioned behaviors) can have complementary strengths and weaknesses that mutually compensate for each other. Coordinated use of these complementary procedures can be more effective than either type of procedure alone in prioritizing compounds for advancement to clinical studies. Drug effects on some of these behaviors in the absence of the pain stimulus can also provide useful information on drug safety to complement data on drug effectiveness.

Third, the progress of preclinical research should build not only on our successes but also on our failures. Inclusion of a positive control (e.g., an opioid or NSAID analgesic) is a common component of experimental design in studies with candidate analgesics and permits direct comparison of effects produced by a test drug with effects of a known analgesic. The recommendation here is to also include a pharmacologically active negative control from among the many candidates that have advanced to clinical testing and failed. For example, many of the studies summarized in this review used centrally acting $\kappa$ opioid receptor agonists as negative controls. This class of drugs is useful as a source of negative controls because $\kappa$ agonists have been extensively evaluated for decades in a wide range of different preclinical procedures, they produce analgesic-like effects in nearly all procedures using pain-stimulated unconditioned behaviors, but no $\kappa$ agonists are approved to treat any type of pain anywhere in the world. Many other types of drug could also be used as a negative control (Yezierski and Hansson, 2018), but 
regardless of the particular drug used, the inclusion of negative as well as positive controls has the value of clarifying dimensions of assay sensitivity and selectivity while providing within-study comparators for use in interpreting effects of candidate analgesics.

The final recommendation focuses not on the conduct of preclinical testing, but on our vocabulary for describing it. Specifically, the labeling of preclinical procedures would benefit from more precise terminology to specify both the putative pain stimulus (the independent variable) and the measured pain behavior (the dependent variable). For example, two procedures commonly used in preclinical research are often labeled as the "hot-plate" and "tail-flick" tests. In the first case, the label names the stimulus but not the behavior. In the second case, the label names the behavior but not the stimulus. More precise labels would name both the stimulus and the behavior using terminology such as "hot-plate paw-withdrawal" and "radiant-heat tail-flick" tests. The importance of this precision is already largely appreciated with regard to the independent "pain-stimulus" variable, and many investigators already use a language that identifies the modality of the stimulus (thermal, mechanical, chemical) and the presence or absence of inflammatory or neuropathic states. However, labeling often omits the name of the behavioral outcome measure, even though this is known to be a determinant of drug effects. Although it is admittedly more cumbersome, disciplined use of such terminology to clarify the behavioral endpoint would emphasize for the investigator and the audience both 1) the specific behavior being measured and 2) the process of inference that is necessarily involved in relating that behavior in animals to the experience of pain in humans. In a corollary to this point, it would be valuable not only to encourage the use of more precise labels for outcome measures but also to discourage (or at least qualify) the use of imprecise labels. In particular, it needs to be emphasized again that terms such as pain, analgesia, allodynia, and hyperalgesia describe patterns of verbal behavior in humans, but verbal behavior is not available as a source of outcome measures in preclinical research with animals. Use of these terms to describe vastly different behaviors in animals invites misunderstanding, complicates translation, and hinders thoughtful consideration of differences in the expression, mechanisms, and pharmacological modulation of the targeted behaviors.

\section{Authorship Contributions}

Wrote or contributed to the writing of the manuscript: Negus.

\section{References}

Akbarali HI and Dewey WL (2017) The gut-brain interaction in opioid tolerance. Curr Opin Pharmacol 37:126-130.

Altarifi AA and Negus SS (2015) Differential tolerance to morphine antinociception in assays of pain-stimulated vs. pain-depressed behavior in rats. Eur J Pharmacol 748:76-82.

Altarifi AA, Rice KC, and Negus SS (2015) Effects of $\mu$-opioid receptor agonists in assays of acute pain-stimulated and pain-depressed behavior in male rats: role of $\mu$-agonist efficacy and noxious stimulus intensity. $J$ Pharmacol Exp Ther $\mathbf{3 5 2}$ 208-217.

Anderson EM, Mills R, Nolan TA, Jenkins AC, Mustafa G, Lloyd C, Caudle RM, and Neubert JK (2013) Use of the Operant Orofacial Pain Assessment Device (OPAD) to measure changes in nociceptive behavior. $J$ Vis Exp 76: e50336.

Andrews N, Legg E, Lisak D, Issop Y, Richardson D, Harper S, Pheby T, Huang W Burgess G, Machin I, et al. (2012) Spontaneous burrowing behaviour in the rat is reduced by peripheral nerve injury or inflammation associated pain. Eur J Pain 16 485-495.

Andrews NA, Latrémolière A, Basbaum AI, Mogil JS, Porreca F, Rice AS, Woolf CJ, Currie GL, Dworkin RH, Eisenach JC, et al. (2016) Ensuring transparency and minimization of methodologic bias in preclinical pain research: PPRECISE considerations. Pain 157:901-909.

Apkarian AV, Bushnell MC, and Schweinhardt P (2013) Representation of pain in the brain, in Wall \& Melzack's Textbook of Pain, 6th ed (McMahon SB, Koltzenburg M, Tracey I, and Turk D, eds) pp 111-128, Elsevier, Philadelphia.

Arendt-Nielsen L, Olesen AE, Staahl C, Menzaghi F, Kell S, Wong GY, and Drewes AM (2009) Analgesic efficacy of peripheral kappa-opioid receptor agonist CR665 compared to oxycodone in a multi-modal, multi-tissue experimental human pain model: selective effect on visceral pain. Anesthesiology 111:616-624.

Armendariz A and Nazarian A (2018) Morphine antinociception on thermal sensitivity and place conditioning in male and female rats treated with intraplantar complete Freund's adjuvant. Behav Brain Res 343:21-27.

Ashrafi F, Mousavi S, and Karimi M (2018) Potential role of bupropion sustained release for cancer-related fatigue: a double-blind, placebo-controlled study. Asian Pac J Cancer Prev 19:1547-1551.

Ator NA and Griffiths RR (2003) Principles of drug abuse liability assessment in laboratory animals. Drug Alcohol Depend 70 (Suppl):S55-S72.

Aviram J and Samuelly-Leichtag G (2017) Efficacy of cannabis-based medicines for pain management: a systematic review and meta-analysis of randomized controlled trials. Pain Physician 20:E755-E796.

Azrin NH, Holz WC, and Hake DF (1963) Fixed-ratio punishment. J Exp Anal Behav 6:141-148.

Babel P, Adamczyk W, Swider K, Bajcar EA, Kicman P, and Lisinska N (2018) How classical conditioning shapes placebo analgesia: hidden versus open conditioning. Pain Med 19:1156-1169.

Backonja MM and Stacey B (2004) Neuropathic pain symptoms relative to overall pain rating. J Pain 5:491-497.

Bagdas D, Muldoon PP, AlSharari S, Carroll FI, Negus SS, and Damaj MI (2016) Expression and pharmacological modulation of visceral pain-induced conditioned place aversion in mice. Neuropharmacology 102:236-243.

Baik JH (2013) Dopamine signaling in reward-related behaviors. Front Neural Circuits 7:152.

Balayssac D, Ling B, Ferrier J, Pereira B, Eschalier A, and Authier N (2014) Assessment of thermal sensitivity in rats using the thermal place preference test: description and application in the study of oxaliplatin-induced acute thermal hypersensitivity and inflammatory pain models. Behav Pharmacol 25:99-111.

Bannister K, Qu C, Navratilova E, Oyarzo J, Xie JY, King T, Dickenson AH, and Porreca F (2017) Multiple sites and actions of gabapentin-induced relief of ongoing experimental neuropathic pain. Pain 158:2386-2395.

Barrett AC, Cook CD, Terner JM, Roach EL, Syvanthong C, and Picker MJ (2002a) Sex and rat strain determine sensitivity to kappa opioid-induced antinociception. Psychopharmacology (Berl) 160:170-181.

Barrett AC, Smith ES, and Picker MJ (2002b) Sex-related differences in mechanical nociception and antinociception produced by mu- and kappa-opioid receptor agonists in rats. Eur $J$ Pharmacol 452:163-173.

Benedetti F (2012) Placebo responses in animals. Pain 153:1983-1984.

Bennett GJ and Xie YK (1988) A peripheral mononeuropathy in rat that produces disorders of pain sensation like those seen in man. Pain 33:87-107.

Berge O-G (2013) Animal models of pain, in Wall and Melzack's Textbook of Pain, 6th ed (McMahon SB, Koltzenburg M, Tracey I, and Turk DC eds) pp 170-182, Elsevier, Philadelphia.

Betourne A, Familiades J, Lacassagne L, Halley H, Cazales M, Ducommun B, Lassalle JM, Zajac JM, and Frances B (2008) Decreased motivational properties of morphine in mouse models of cancerous- or inflammatory-chronic pain: implication of supraspinal neuropeptide FF(2) receptors. Neuroscience 157:12-21.

Boada MD, Martin TJ, and Ririe DG (2016) Nerve injury induced activation of fastconducting high threshold mechanoreceptors predicts non-reflexive pain related behavior. Neurosci Lett 632:44-49.

Borsook D, Becerra L, Carlezon WA Jr, Shaw M, Renshaw P, Elman I, and Levine J (2007) Reward-aversion circuitry in analgesia and pain: implications for psychiatric disorders. Eur J Pain 11:7-20.

Boyette-Davis JA, Thompson CD, and Fuchs PN (2008) Alterations in attentiona mechanisms in response to acute inflammatory pain and morphine administration. Neuroscience 151:558-563.

Bray GE, Ying Z, Baillie LD, Zhai R, Mulligan SJ, and Verge VM (2013) Extracellular $\mathrm{pH}$ and neuronal depolarization serve as dynamic switches to rapidly mobilize trkA to the membrane of adult sensory neurons. J Neurosci 33:8202-8215.

Bridges D, Ahmad K, and Rice AS (2001) The synthetic cannabinoid WIN55,212-2 attenuates hyperalgesia and allodynia in a rat model of neuropathic pain. $\mathrm{Br} J$ Pharmacol 133:586-594.

Brown DC, Boston RC, Coyne JC, and Farrar JT (2008) Ability of the canine brief pain inventory to detect response to treatment in dogs with osteoarthritis. J Am Vet Med Assoc 233:1278-1283.

Brust TF, Morgenweck J, Kim SA, Rose JH, Locke JL, Schmid CL, Zhou L, Stah EL, Cameron MD, Scarry SM, et al. (2016) Biased agonists of the kappa opioid receptor suppress pain and itch without causing sedation or dysphoria. Sci Signal 9:ra117. 
Cahill CM, Xue L, Grenier P, Magnussen C, Lecour S, and Olmstead MC (2013) Changes in morphine reward in a model of neuropathic pain. Behav Pharmacol 24: 207-213.

Carter LP and Griffiths RR (2009) Principles of laboratory assessment of drug abuse liability and implications for clinical development. Drug Alcohol Depend 105 (Suppl 1):S14-S25.

Chambers CT, Finley GA, McGrath PJ, and Walsh TM (2003) The parents' postoperative pain measure: replication and extension to 2-6-year-old children. Pain 105:437-443

Chambers CT, Reid GJ, McGrath PJ, and Finley GA (1996) Development and preliminary validation of a postoperative pain measure for parents. Pain 68:307-313. Chaplan SR, Bach FW, Pogrel JW, Chung JM, and Yaksh TL (1994) Quantitative assessment of tactile allodynia in the rat paw. J Neurosci Methods 53:55-63.

Chapman CR, Lipschitz DL, Angst MS, Chou R, Denisco RC, Donaldson GW, Fine PG, Foley KM, Gallagher RM, Gilson AM, et al. (2010) Opioid pharmacotherapy for chronic non-cancer pain in the United States: a research guideline for developing an evidence-base. J Pain 11:807-829.

Che T, Majumdar S, Zaidi SA, Ondachi P, McCorvy JD, Wang S, Mosier PD, Uprety R, Vardy E, Krumm BE, et al. (2018) Structure of the nanobody-stabilized active state of the kappa opioid receptor. Cell 172:55-67.e15.

Clark JD (2016) Preclinical pain research: can we do better? Anesthesiology 125:846-849.

Cobos EJ, Ghasemlou N, Araldi D, Segal D, Duong K, and Woolf CJ (2012) Inflammation-induced decrease in voluntary wheel running in mice: a nonreflexive test for evaluating inflammatory pain and analgesia. Pain 153:876-884

Colpaert FC (1999) Drug discrimination in neurobiology. Pharmacol Biochem Behav 64:337-345.

Colpaert FC, De Witte P, Maroli AN, Awouters F, Niemegeers CJ, and Janssen PA (1980) Self-administration of the analgesic suprofen in arthritic rats: evidence of Mycobacterium butyricum-induced arthritis as an experimental model of chronic pain. Life Sci 27:921-928.

Colpaert FC, Meert T, De Witte P, and Schmitt P (1982) Further evidence validating adjuvant arthritis as an experimental model of chronic pain in the rat. Life Sci 31:67-75.

Colpaert FC, Tarayre JP, Alliaga M, Bruins Slot LA, Attal N, and Koek W (2001) Opiate self-administration as a measure of chronic nociceptive pain in arthritic rats. Pain 91:33-45.

Cone K, Lanpher J, Kinens A, Richard P, Couture S, Brackin R, Payne E, Harrington K, Rice KC, and Stevenson GW (2018) Delta/mu opioid receptor interactions in operant conditioning assays of pain-depressed responding and drug-induced rate suppression: assessment of therapeutic index in male Sprague Dawley rats. Psychopharmacology 235:1609-1618.

Conti S, Costa B, Colleoni M, Parolaro D, and Giagnoni G (2002) Antiinflammatory action of endocannabinoid palmitoylethanolamide and the synthetic cannabinoid nabilone in a model of acute inflammation in the rat. $\mathrm{Br} J$ Pharmacol 135 181-187.

Corder G, Ahanonu B, Grewe BF, Wang D, Schnitzer MJ, and Scherrer G (2019) An amygdalar neural ensemble that encodes the unpleasantness of pain. Science $\mathbf{3 6 3}$ : $276-281$.

Craft RM, Wakley AA, Tsutsui KT, and Laggart JD (2012) Sex differences in cannabinoid 1 vs. cannabinoid 2 receptor-selective antagonism of antinociception produced by delta9-tetrahydrocannabinol and CP55,940 in the rat. J Pharmacol Exp Ther 340:787-800.

Curzon P, Rustay NR, and Browman KE (2009) Cued and contextual fear conditioning for rodents, in Methods of Behavior Analysis in Neuroscience, 2nd ed (Buccafusco JJ ed) CRC Press, Boca Raton, FL.

Deval E, Gasull X, Noël J, Salinas M, Baron A, Diochot S, and Lingueglia E (2010) Acid-sensing ion channels (ASICs): pharmacology and implication in pain. Pharmacol Ther 128:549-558.

Deyama S, Katayama T, Ohno A, Nakagawa T, Kaneko S, Yamaguchi T, Yoshioka M, and Minami M (2008) Activation of the beta-adrenoceptor-protein kinase A signaling pathway within the ventral bed nucleus of the stria terminalis mediates the negative affective component of pain in rats. $J$ Neurosci 28:7728-7736.

Deyama S, Nakagawa T, Kaneko S, Uehara T, and Minami M (2007) Involvement of the bed nucleus of the stria terminalis in the negative affective component of visceral and somatic pain in rats. Behav Brain Res 176:367-371.

Deyo RA, Dworkin SF, Amtmann D, Andersson G, Borenstein D, Carragee E, Carrino J, Chou R, Cook K, DeLitto A, et al. (2014) Report of the NIH Task Force on research standards for chronic low back pain. J Pain 15:569-585.

Di Marzo V and Petrocellis LD (2006) Plant, synthetic, and endogenous cannabinoids in medicine. Annu Rev Med 57:553-574.

Donvito G, Nass SR, Wilkerson JL, Curry ZA, Schurman LD, Kinsey SG, and Lichtman $\mathrm{AH}$ (2018) The endogenous cannabinoid system: a budding source of targets for treating inflammatory and neuropathic pain. Neuropsychopharmacology 43:52-79.

Dworkin RH, Turk DC, Farrar JT, Haythornthwaite JA, Jensen MP, Katz NP, Kerns RD, Stucki G, Allen RR, Bellamy N, et al.; IMMPACT (2005) Core outcome measures for chronic pain clinical trials: IMMPACT recommendations. Pain 113:9-19.

Ehrich JM, Messinger DI, Knakal CR, Kuhar JR, Schattauer SS, Bruchas MR, Zweifel LS, Kieffer BL, Phillips PE, and Chavkin C (2015) Kappa opioid receptorinduced aversion requires p38 MAPK activation in VTA dopamine neurons. $J$ Neurosci 35:12917-12931.

Endoh T, Matsuura H, Tajima A, Izumimoto N, Tajima C, Suzuki T, Saitoh A, Suzuki T, Narita M, Tseng L, et al. (1999) Potent antinociceptive effects of TRK-820, a novel kappa-opioid receptor agonist. Life Sci 65:1685-1694.

Endoh T, Tajima A, Izumimoto N, Suzuki T, Saitoh A, Suzuki T, Narita M, Kamei J, Tseng LF, Mizoguchi H, et al. (2001) TRK-820, a selective kappa-opioid agonist, produces potent antinociception in cynomolgus monkeys. Jpn J Pharmacol 85 $282-290$

Endoh T, Tajima A, Suzuki T, Kamei J, Narita M, Tseng L, and Nagase H (2000) Characterization of the antinociceptive effects of TRK- 820 in the rat. Eur $J$ Pharmacol 387:133-140
Erichsen HK and Blackburn-Munro G (2002) Pharmacological characterisation of the spared nerve injury model of neuropathic pain. Pain 98:151-161.

Evenden J, Ross L, Jonak G, and Zhou J (2009) A novel operant conflict procedure using incrementing shock intensities to assess the anxiolytic and anxiogenic effects of drugs. Behav Pharmacol 20:226-236.

Ewan EE and Martin TJ (2011) Opioid facilitation of rewarding electrical brain stimulation is suppressed in rats with neuropathic pain. Anesthesiology 114: 624-632.

Ewan EE and Martin TJ (2014) Differential suppression of intracranial selfstimulation, food-maintained operant responding, and open field activity by paw incision and spinal nerve ligation in rats. Anesth Analg 118:854-862.

Fallon MT, Albert Lux E, McQuade R, Rossetti S, Sanchez R, Sun W, Wright S, Lichtman AH, and Kornyeyeva E (2017) Sativex oromucosal spray as adjunctive therapy in advanced cancer patients with chronic pain unalleviated by optimized opioid therapy: two double-blind, randomized, placebo-controlled phase 3 studies. Br J Pain 11:119-133.

Fan F, Compton DR, Ward S, Melvin L, and Martin BR (1994) Development of crosstolerance between delta 9-tetrahydrocannabinol, CP 55,940 and WIN 55,212. $J$ Pharmacol Exp Ther 271:1383-1390.

Fanselow MS and Wassum KM (2015) The origins and organization of vertebrate pavlovian conditioning. Cold Spring Harb Perspect Biol 8:a021717.

Fearon A, Neeman T, Smith P, Scarvell J, and Cook J (2017) Pain, not structural impairments may explain activity limitations in people with gluteal tendinopathy or hip osteoarthritis: a cross sectional study. Gait Posture 52:237-243.

Field MJ, Carnell AJ, Gonzalez MI, McCleary S, Oles RJ, Smith R, Hughes J, and Singh L (1999) Enadoline, a selective kappa-opioid receptor agonist shows potent antihyperalgesic and antiallodynic actions in a rat model of surgical pain. Pain 80:383-389.

Finnerup NB, Attal N, Haroutounian S, McNicol E, Baron R, Dworkin RH, Gilron I, Haanpää M, Hansson P, Jensen TS, et al. (2015) Pharmacotherapy for neuropathic pain in adults: a systematic review and meta-analysis. Lancet Neurol 14:162-173.

Foley KM (1995) Misconceptions and controversies regarding the use of opioids in cancer pain. Anticancer Drugs 6 (Suppl 3):4-13.

Forte BL, Slosky LM, Zhang H, Arnold MR, Staatz WD, Hay M, Largent-Milnes TM, and Vanderah TW (2016) Angiotensin-(1-7)/Mas receptor as an antinociceptive agent in cancer-induced bone pain. Pain 157:2709-2721.

Freitas KC, Hillhouse TM, Leitl MD, and Negus SS (2015) Effects of acute and sustained pain manipulations on performance in a visual-signal detection task of attention in rats. Drug Dev Res 76:194-203.

Fuchs PN and McNabb CT (2012) The place escape/avoidance paradigm: a novel method to assess nociceptive processing. $J$ Integr Neurosci 11:61-72.

Gaskill BN, Karas AZ, Garner JP, and Pritchett-Corning KR (2013) Nest building as an indicator of health and welfare in laboratory mice. $J$ Vis Exp 82:51012.

Gerrig R and Zimbardo P (2002) Psychology and Life, Allyn and Bacon, Boston.

Gould SA, Doods H, Lamla T, and Pekcec A (2016) Pharmacological characterization of intraplantar complete Freund's adjuvant-induced burrowing deficits. Behav Brain Res 301:142-151.

Grace PM, Strand KA, Maier SF, and Watkins LR (2014) Suppression of voluntary wheel running in rats is dependent on the site of inflammation: evidence for voluntary running as a measure of hind paw-evoked pain. J Pain 15:121-128.

Greene CS, Goddard G, Macaluso GM, and Mauro G (2009) Topical review: placebo responses and therapeutic responses. How are they related? J Orofac Pain 23 : 93-107.

Greenwald MK and Stitzer ML (2000) Antinociceptive, subjective and behavioral effects of smoked marijuana in humans. Drug Alcohol Depend 59:261-275.

Grubb BD (2004) Activation of sensory neurons in the arthritic joint. Novartis Found Symp 260:28-36; discussion 36-48, 100-104, 277-279.

Haber SN (2014) The place of dopamine in the cortico-basal ganglia circuit. Neuroscience 282:248-257.

Hargreaves K, Dubner R, Brown F, Flores C, and Joris J (1988) A new and sensitive method for measuring thermal nociception in cutaneous hyperalgesia. Pain 32 . $77-88$.

Harte SE, Meyers JB, Donahue RR, Taylor BK, and Morrow TJ (2016) Mechanical conflict system: a novel operant method for the assessment of nociceptive behavior. PLoS One 11:e150164.

Harton LR, Richardson JR, Armendariz A, and Nazarian A (2017) Dissociation of morphine analgesic effects in the sensory and affective components of formalininduced spontaneous pain in male and female rats. Brain Res 1658:36-41.

Harvie DS, Moseley GL, Hillier SL, and Meulders A (2017) Classical conditioning differences associated with chronic pain: a systematic review. J Pain 18:889-898.

Havelin J, Imbert I, Sukhtankar D, Remeniuk B, Pelletier I, Gentry J, Okun A Tiutan T, Porreca F, and King TE (2017) Mediation of movement-induced breakthrough cancer pain by IB4-binding nociceptors in rats. J Neurosci $\mathbf{3 7}$ 5111-5122.

Herrera C, Bolton F, Arias AS, Harrison RA, and Gutiérrez JM (2018) Analgesic effect of morphine and tramadol in standard toxicity assays in mice injected with venom of the snake Bothrops asper. Toxicon 154:35-41.

Hill R (2000) NK1 (substance P) receptor antagonists--why are they not analgesic in humans? Trends Pharmacol Sci 21:244-246.

Huggins JP, Smart TS, Langman S, Taylor L, and Young T (2012) An efficient randomised, placebo-controlled clinical trial with the irreversible fatty acid amide hydrolase-1 inhibitor PF-04457845, which modulates endocannabinoids but fails to induce effective analgesia in patients with pain due to osteoarthritis of the knee. Pain 153:1837-1846.

Hughes FM Jr, Shaner BE, Brower JO, Woods RJ, and Dix TA (2013) Development of a Peptide-derived orally-active kappa-opioid receptor agonist targeting peripheral pain. Open Med Chem J 7:16-22.

Hummel M, Lu P, Cummons TA, and Whiteside GT (2008) The persistence of a longterm negative affective state following the induction of either acute or chronic pain. Pain 140:436-445. 
Hung CY and Tan CH (2018) TRP channels in nociception and pathological pain. Adv Exp Med Biol 1099:13-27.

Hunter JC, Leighton GE, Meecham KG, Boyle SJ, Horwell DC, Rees DC, and Hughes J (1990) CI-977, a novel and selective agonist for the kappa-opioid receptor. $\mathrm{Br} J$ Pharmacol 101:183-189.

Ikemoto S (2010) Brain reward circuitry beyond the mesolimbic dopamine system: a neurobiological theory. Neurosci Biobehav Rev 35:129-150.

Institute of Medicine Committee on Advancing Pain Research, Care, and Education (2011) Relieving Pain in America: A Blueprint for Transforming Prevention, Care, Education, and Research, National Academies Press, Washington, DC.

International Association for the Study of Pain (1994) Part III. Pain terms, a current list with definitions and notes on usage, in Classification of Chronic Pain (Merskey $\mathrm{H}$ and Bogduk N eds) pp 209-214, IASP Press, Seattle, WA.

Inui S (2015) Nalfurafine hydrochloride to treat pruritus: a review. Clin Cosmet Investig Dermatol 8:249-255.

Jamison RN and Mao J (2015) Opioid analgesics. Mayo Clin Proc 90:957-968.

Jensen K, Kirsch I, Odmalm S, Kaptchuk TJ, and Ingvar M (2015) Classical conditioning of analgesic and hyperalgesic pain responses without conscious awareness. Proc Natl Acad Sci USA 112:7863-7867.

Jirkof P (2014) Burrowing and nest building behavior as indicators of well-being in mice. J Neurosci Methods 234:139-146.

Johansen JP, Fields HL, and Manning BH (2001) The affective component of pain in rodents: direct evidence for a contribution of the anterior cingulate cortex. Proc Natl Acad Sci USA 98:8077-8082.

Jones MR, Kaye AD, Kaye AJ, and Urman RD (2016) The emerging therapeutic roles of к-opioid agonists. J Opioid Manag 12:101-107.

Joshi SK and Honore P (2006) Animal models of pain for drug discovery. Expert Opin Drug Discov 1:323-334.

Kanavaki AM, Rushton A, Efstathiou N, Alrushud A, Klocke R, Abhishek A, and Duda JL (2017) Barriers and facilitators of physical activity in knee and hip osteoarthritis: a systematic review of qualitative evidence. BMJ Open 7 : e017042

Kandasamy R, Calsbeek JJ, and Morgan MM (2016) Home cage wheel running is an objective and clinically relevant method to assess inflammatory pain in male and female rats. J Neurosci Methods 263:115-122.

Kandasamy R, Calsbeek JJ, and Morgan MM (2017) Analysis of inflammationinduced depression of home cage wheel running in rats reveals the difference between opioid antinociception and restoration of function. Behav Brain Res 317: 502-507.

Kandasamy R, Dawson CT, Craft RM, and Morgan MM (2018) Anti-migraine effect of $\Delta^{9}$-tetrahydrocannabinol in the female rat. Eur J Pharmacol 818:271-277.

Kang SJ, Kim S, Lee J, Kwak C, Lee K, Zhuo M, and Kaang BK (2017) Inhibition of anterior cingulate cortex excitatory neuronal activity induces conditioned place preference in a mouse model of chronic inflammatory pain. Korean $J$ Physiol Pharmacol 21:487-493.

Kangas BD and Bergman J (2014) Operant nociception in nonhuman primates. Pain 155:1821-1828.

Keeler JF and Robbins TW (2011) Translating cognition from animals to humans. Biochem Pharmacol 81:1356-1366.

Kenakin T (2015) The effective application of biased signaling to new drug discovery. Mol Pharmacol 88:1055-1061.

Kim TK (2017) Practical statistics in pain research. Korean J Pain 30:243-249.

King T, Vera-Portocarrero L, Gutierrez T, Vanderah TW, Dussor G, Lai J, Fields HL, and Porreca F (2009) Unmasking the tonic-aversive state in neuropathic pain. Nat Neurosci 12:1364-1366.

Kinsey SG, Mahadevan A, Zhao B, Sun H, Naidu PS, Razdan RK, Selley DE, Imad Damaj M, and Lichtman AH (2011) The CB2 cannabinoid receptor-selective agonist $\mathrm{O}-3223$ reduces pain and inflammation without apparent cannabinoid behavioral effects. Neuropharmacology 60:244-251.

Klinck MP, Mogil JS, Moreau M, Lascelles BDX, Flecknell PA, Poitte T, and Troncy E (2017) Translational pain assessment: could natural animal models be the missing link? Pain 158:1633-1646.

Kostek M, Polaski A, Kolber B, Ramsey A, Kranjec A, and Szucs K (2016) A protocol of manual tests to measure sensation and pain in humans. J Vis Exp 118: 54130

Kraft B, Frickey NA, Kaufmann RM, Reif M, Frey R, Gustorff B, and Kress HG (2008) Lack of analgesia by oral standardized cannabis extract on acute inflammatory pain and hyperalgesia in volunteers. Anesthesiology 109:101-110.

Kristensen PJ, Heegaard AM, Hestehave S, Jeggo RD, Bjerrum OJ, and Munro G (2017) Vendor-derived differences in injury-induced pain phenotype and pharmacology of Sprague-Dawley rats: does it matter? Eur J Pain 21:692-704.

Kwilasz AJ and Negus SS (2012) Dissociable effects of the cannabinoid receptor agonists $\Delta 9$-tetrahydrocannabinol and CP55940 on pain-stimulated versus paindepressed behavior in rats. $J$ Pharmacol Exp Ther 343:389-400.

LaBuda CJ and Fuchs PN (2000) Morphine and gabapentin decrease mechanical hyperalgesia and escape/avoidance behavior in a rat model of neuropathic pain. Neurosci Lett 290:137-140.

Langford DJ, Bailey AL, Chanda ML, Clarke SE, Drummond TE, Echols S, Glick S, Ingrao J, Klassen-Ross T, Lacroix-Fralish ML, et al. (2010) Coding of facial expressions of pain in the laboratory mouse. Nat Methods 7:447-449.

Lazenka ML, Moerke MJ, Townsend EA, Freeman KB, Carroll FI, and Negus SS (2018) Dissociable effects of the kappa opioid receptor agonist nalfurafine on pain/ itch-stimulated and pain/itch-depressed behaviors in male rats. Psychopharmacology (Berl) 235:203-213.

Le Bars D, Hansson PT, and Plaghki L (2010) Current animal tests and models of pain, in Pharmacology of Pain (Beaulieu P, Lussier D, Porreca F, and Dickenson AH eds) pp 475-504, IASP Press, Seattle, WA

Lee JH and Stitzer ML (1995) A novel radiant heat test for assessing pain threshold in human subjects: measurement stability. Behav Res Methods Instrum Comput 27:41-45.
Legakis LP, Bigbee JW, and Negus SS (2018) Lack of paclitaxel effects on intracranial self-stimulation in male and female rats: comparison to mechanica sensitivity. Behav Pharmacol 29:290-298.

Leitl MD and Negus SS (2016) Pharmacological modulation of neuropathic painrelated depression of behavior: effects of morphine, ketoprofen, bupropion and [INCREMENT]9-tetrahydrocannabinol on formalin-induced depression of intracranial self-stimulation in rats. Behav Pharmacol 27:364-376.

Leitl MD, Onvani S, Bowers MS, Cheng K, Rice KC, Carlezon WA Jr, Banks ML, and Negus SS (2014) Pain-related depression of the mesolimbic dopamine system in rats: expression, blockade by analgesics, and role of endogenous к-opioids. Neuropsychopharmacology 39:614-624.

Leshner AI and Koob GF (1999) Drugs of abuse and the brain. Proc Assoc Am Physicians 111:99-108.

Lewter LA, Fisher JL, Siemian JN, Methuku KR, Poe MM, Cook JM, and Li JX (2017) Antinociceptive effects of a novel $\alpha 2 / \alpha 3$-subtype selective $\mathrm{GABA}_{\mathrm{A}}$ receptor positive allosteric modulator. ACS Chem Neurosci 8:1305-1312.

Li J, Daughters RS, Bullis C, Bengiamin R, Stucky MW, Brennan J, and Simone DA (1999) The cannabinoid receptor agonist WIN 55,212-2 mesylate blocks the development of hyperalgesia produced by capsaicin in rats. Pain 81:25-33.

Li JX (2013) The application of conditioning paradigms in the measurement of pain. Eur J Pharmacol 716:158-168.

Lin JY, Arthurs J, and Reilly S (2017) Conditioned taste aversions: from poisons to pain to drugs of abuse. Psychon Bull Rev 24:335-351.

Lomas LM, Barrett AC, Terner JM, Lysle DT, and Picker MJ (2007) Sex differences in the potency of kappa opioids and mixed-action opioids administered systemically and at the site of inflammation against capsaicin-induced hyperalgesia in rats. Psychopharmacology (Berl) 191:273-285.

Machelska H, Pflüger M, Weber W, Piranvisseh-Völk M, Daubert JD, Dehaven R, and Stein C (1999) Peripheral effects of the kappa-opioid agonist EMD 61753 on pain and inflammation in rats and humans. $J$ Pharmacol Exp Ther 290:354-361.

Maciel AA, Cunha PR, Laraia IO, and Trevisan F (2014) Efficacy of gabapentin in the improvement of pruritus and quality of life of patients with notalgia paresthetica. An Bras Dermatol 89:570-575.

Madden V.J, Harvie DS, Parker R, Jensen KB, Vlaeyen JW, Moseley GL, and Stanton TR (2016) Can pain or hyperalgesia Be a classically conditioned response in humans? A systematic review and meta-analysis. Pain Med 17:1094-1111.

Manchikanti L, Helm S 2nd, Fellows B, Janata JW, Pampati V, Grider JS, and Boswell MV (2012) Opioid epidemic in the United States. Pain Physician 15 (Suppl):ES9-ES38.

Mao J, Price DD, Lu J, Keniston L, and Mayer DJ (2000) Two distinctive antinociceptive systems in rats with pathological pain. Neurosci Lett 280:13-16.

Martikainen IK, Hagelberg N, Jääskeläinen SK, Hietala J, and Pertovaara A (2018) Dopaminergic and serotonergic mechanisms in the modulation of pain: in vivo studies in human brain. Eur J Pharmacol 834:337-345

Martin BR and Lichtman AH (1998) Cannabinoid transmission and pain perception. Neurobiol Dis 5:447-461.

Martin TJ, Buechler NL, Kahn W, Crews JC, and Eisenach JC (2004) Effects of laparotomy on spontaneous exploratory activity and conditioned operant responding in the rat: a model for postoperative pain. Anesthesiology 101:191-203.

Martin TJ and Ewan E (2008) Chronic pain alters drug self-administration: implications for addiction and pain mechanisms. Exp Clin Psychopharmacol 16: $357-366$.

Martin TJ, Kim SA, Buechler NL, Porreca F, and Eisenach JC (2007) Opioid selfadministration in the nerve-injured rat: relevance of antiallodynic effects to drug consumption and effects of intrathecal analgesics. Anesthesiology 106:312-322.

Martin TJ, Kim SA, and Eisenach JC (2006) Clonidine maintains intrathecal selfadministration in rats following spinal nerve ligation. Pain 125:257-263.

Martin TJ, Strassburg TJ, Grigg AL, Kim SA, Ririe DG, and Eisenach JC (2017) Assessment of behavioral disruption in rats with abdominal inflammation using visual cue titration and the five-choice serial-reaction time task. Anesthesiology 127:372-381.

Maruyama C, Deyama S, Nagano Y, Ide S, Kaneda K, Yoshioka M, and Minami M (2018) Suppressive effects of morphine injected into the ventral bed nucleus of the stria terminalis on the affective, but not sensory, component of pain in rats. Eur $J$ Neurosci 47:40-47.

Matson DJ, Broom DC, Carson SR, Baldassari J, Kehne J, and Cortright DN (2007) Inflammation-induced reduction of spontaneous activity by adjuvant: a novel model to study the effect of analgesics in rats. J Pharmacol Exp Ther 320:194-201.

Matson DJ, Broom DC, and Cortright DN (2010) Locomotor activity in a novel environment as a test of inflammatory pain in rats. Methods Mol Biol 617:67-78.

Mauderli AP, Acosta-Rua A, and Vierck CJ (2000) An operant assay of thermal pain in conscious, unrestrained rats. $J$ Neurosci Methods 97:19-29.

Mazza S, Frot M, and Rey AE (2018) A comprehensive literature review of chronic pain and memory. Prog Neuropsychopharmacol Biol Psychiatry 87:183-192.

McGrath PJ and Unruh AM (2013) Measurement and assessment of pediatric pain, in Wall and Melzack's Textbook of Pain, 6th ed (McMahon SB, Koltzenburg M, Tracey I, and Turk DC eds) pp 320-327, Elseivier, Philadelphia.

McNabb CT, White MM, Harris AL, and Fuchs PN (2014) The elusive rat model of conditioned placebo analgesia. Pain 155:2022-2032.

Melzack R and Katz J (2013) Pain measurement in adult patients, in Wall and Melzack's Textbook of Pain, 6th ed (McMahon SB, Koltzenburg M, Tracey I, and Turk DC eds) pp 301-319, Elsevier, Philadelphia.

Millan MJ (1990) Kappa-opioid receptors and analgesia. Trends Pharmacol Sci 11 $70-76$

Miller LL, Leitl MD, Banks ML, Blough BE, and Negus SS (2015) Effects of the triple monoamine uptake inhibitor amitifadine on pain-related depression of behavior and mesolimbic dopamine release in rats. Pain 156:175-184.

Miller LL, Picker MJ, Schmidt KT, and Dykstra LA (2011) Effects of morphine on pain-elicited and pain-suppressed behavior in CB1 knockout and wildtype mice. Psychopharmacology (Berl) 215:455-465. 
Miller LL, Picker MJ, Umberger MD, Schmidt KT, and Dykstra LA (2012) Effects of alterations in cannabinoid signaling, alone and in combination with morphine, on painelicited and pain-suppressed behavior in mice. J Pharmacol Exp Ther 342:177-187.

Mogenson GJ, Jones DL, and Yim CY (1980) From motivation to action: functional interface between the limbic system and the motor system. Prog Neurobiol 14: 69-97.

Mogil JS (2009) Animal models of pain: progress and challenges. Nat Rev Neurosci 10:283-294.

Montagne-Clavel J and Oliveras JL (1996) The "plantar test" apparatus (Ugo Basile Biological Apparatus), a controlled infrared noxious radiant heat stimulus for precise withdrawal latency measurement in the rat, as a tool for humans? Somatosens Mot Res 13:215-223.

Morgan D, Cook CD, Smith MA, and Picker MJ (1999) An examination of the interactions between the antinociceptive effects of morphine and various mu-opioids the role of intrinsic efficacy and stimulus intensity. Anesth Analg 88:407-413.

Mori T, Kanbara T, Harumiya M, Iwase Y, Masumoto A, Komiya S, Nakamura A Shibasaki M, Kanemasa T, Sakaguchi G, et al. (2014) Establishment of opioidinduced rewarding effects under oxaliplatin- and Paclitaxel-induced neuropathy in rats. J Pharmacol Sci 126:47-55.

Morris RG, Davis S, and Butcher SP (1990) Hippocampal synaptic plasticity and NMDA receptors: a role in information storage? Philos Trans $R$ Soc Lond B Biol Sci 329:187-204

Moss DE and Johnson RL (1980) Tonic analgesic effects of delta 9-tetrahydrocannabinol as measured with the formalin test. Eur J Pharmacol 61:313-315.

Mücke M, Phillips T, Radbruch L, Petzke F, and Häuser W (2018) Cannabis-based medicines for chronic neuropathic pain in adults. Cochrane Database Syst Rev $\mathbf{3}$ CD012182

Munro G, Jansen-Olesen I, and Olesen J (2017) Animal models of pain and migraine in drug discovery. Drug Discov Today 22:1103-1111.

Murphy NP, Mills RH, Caudle RM, and Neubert JK (2014) Operant assays for assessing pain in preclinical rodent models: highlights from an orofacial assay. Curr Top Behav Neurosci 20:121-145.

Nagase H, Hayakawa J, Kawamura K, Kawai K, Takezawa Y, Matsuura H, Tajima C, and Endo T (1998) Discovery of a structurally novel opioid kappa-agonist derived from 4,5-epoxymorphinan. Chem Pharm Bull (Tokyo) 46:366-369.

Nakamura A, Narita M, Miyoshi K, Shindo K, Okutsu D, Suzuki M, Higashiyama K, and Suzuki T (2008) Changes in the rewarding effects induced by tramadol and its active metabolite M1 after sciatic nerve injury in mice. Psychopharmacology (Berl) 200:307-316

National Academies of Sciences Engineering and Medicine (2017) The Health Effects of Cannabis and Cannabinoids: The Current State of Evidence and Recommendations for Research, National Academies Press, Washington, DC.

National Research Council (2011) Guide for the Care and Use of Laboratory Animals, National Academies Press, Washington, DC.

Navratilova E, Morimura K, Xie JY, Atcherley CW, Ossipov MH, and Porreca F (2016) Positive emotions and brain reward circuits in chronic pain. J Comp Neurol 524:1646-1652.

Navratilova E, Xie JY, Meske D, Qu C, Morimura K, Okun A, Arakawa N, Ossipov M, Fields HL, and Porreca F (2015) Endogenous opioid activity in the anterior cingulate cortex is required for relief of pain. J Neurosci 35:7264-7271.

Nazemi S, Manaheji H, Zaringhalam J, Sadeghi M, and Haghparast A (2012) Postinjury repeated administrations of minocycline improve the antinociceptive effect of morphine in chronic constriction injury model of neuropathic pain in rat. Pharmacol Biochem Behav 102:520-525.

Neelakantan H, Tallarida RJ, Reichenbach ZW, Tuma RF, Ward SJ, and Walker EA (2015) Distinct interactions of cannabidiol and morphine in three nociceptive behavioral models in mice. Behav Pharmacol 26:304-314.

Neelakantan H, Ward SJ, and Walker EA (2016) Effects of paclitaxel on mechanical sensitivity and morphine reward in male and female C57Bl6 mice. Exp Clin Psychopharmacol 24:485-495.

Negus SS (2013) Expression and treatment of pain-related behavioral depression. Lab Anim (NY) 42:292-300.

Negus SS (2018) Addressing the opioid crisis: the importance of choosing translational endpoints in analgesic drug discovery. Trends Pharmacol Sci 39:327-330.

Negus SS, Bilsky EJ, Do Carmo GP, and Stevenson GW (2010a) Rationale and methods for assessment of pain-depressed behavior in preclinical assays of pain and analgesia. Methods Mol Biol 617:79-91.

Negus SS and Miller LL (2014) Intracranial self-stimulation to evaluate abuse potential of drugs. Pharmacol Rev 66:869-917.

Negus SS, Morrissey EM, Rosenberg M, Cheng K, and Rice KC (2010b) Effects of kappa opioids in an assay of pain-depressed intracranial self-stimulation in rats. Psychopharmacology (Berl) 210:149-159.

Negus SS, Neddenriep B, Altarifi AA, Carroll FI, Leitl MD, and Miller LL (2015) Effects of ketoprofen, morphine, and kappa opioids on pain-related depression of nesting in mice. Pain 156:1153-1160.

Negus SS, O'Connell R, Morrissey E, Cheng K, and Rice KC (2012) Effects of peripherally restricted $\kappa$ opioid receptor agonists on pain-related stimulation and depression of behavior in rats. J Pharmacol Exp Ther 340:501-509.

Negus SS, Vanderah TW, Brandt MR, Bilsky EJ, Becerra L, and Borsook D (2006) Preclinical assessment of candidate analgesic drugs: recent advances and future challenges. J Pharmacol Exp Ther 319:507-514.

Neill JC, Barnes S, Cook S, Grayson B, Idris NF, McLean SL, Snigdha S, Rajagopal L, and Harte MK (2010) Animal models of cognitive dysfunction and negative symptoms of schizophrenia: focus on NMDA receptor antagonism. Pharmacol Ther 128:419-432

Nestler EJ and Carlezon WA Jr (2006) The mesolimbic dopamine reward circuit in depression. Biol Psychiatry 59:1151-1159.

Neubert JK, King C, Malphurs W, Wong F, Weaver JP, Jenkins AC, Rossi HL, and Caudle RM (2008) Characterization of mouse orofacial pain and the effects of lesioning TRPV1-expressing neurons on operant behavior. Mol Pain 4:43.
Neubert JK, Rossi HL, Malphurs W, Vierck CJ Jr, and Caudle RM (2006) Differentiation between capsaicin-induced allodynia and hyperalgesia using a therma operant assay. Behav Brain Res 170:308-315.

Neubert JK, Rossi HL, Pogar J, Jenkins AC, and Caudle RM (2007) Effects of muand kappa-2 opioid receptor agonists on pain and rearing behaviors. Behav Brain Funct 3:49.

Neubert JK, Widmer CG, Malphurs W, Rossi HL, Vierck CJ Jr, and Caudle RM (2005) Use of a novel thermal operant behavioral assay for characterization of orofacial pain sensitivity. Pain 116:386-395.

Niikura K, Narita M, Narita M, Nakamura A, Okutsu D, Ozeki A, Kurahashi K, Kobayashi Y, Suzuki M, and Suzuki T (2008) Direct evidence for the involvement of endogenous beta-endorphin in the suppression of the morphine-induced rewarding effect under a neuropathic pain-like state. Neurosci Lett 435:257-262.

O'Connor EC, Chapman K, Butler P, and Mead AN (2011) The predictive validity of the rat self-administration model for abuse liability. Neurosci Biobehav Rev 35: 912-938.

Okun A, McKinzie DL, Witkin JM, Remeniuk B, Husein O, Gleason SD, Oyarzo J, Navratilova E, McElroy B, Cowen S, et al. (2016) Hedonic and motivational responses to food reward are unchanged in rats with neuropathic pain. Pain 157: 2731-2738.

Oliver VL, Thurston SE, and Lofgren JL (2018) Using cageside measures to evaluate analgesic efficacy in mice (Mus musculus) after surgery. J Am Assoc Lab Anim Sci 57:186-201.

Overton DA (1988) Similarities and differences between behavioral control by drugproduced stimuli and by sensory stimuli. Psychopharmacol Ser 4:176-198.

Ozaki S, Narita M, Narita M, Iino M, Sugita J, Matsumura Y, and Suzuki T (2002) Suppression of the morphine-induced rewarding effect in the rat with neuropathic pain: implication of the reduction in mu-opioid receptor functions in the ventra tegmental area. J Neurochem 82:1192-1198.

Pachman DR, Barton DL, Watson JC, and Loprinzi CL (2011) Chemotherapyinduced peripheral neuropathy: prevention and treatment. Clin Pharmacol Ther 90:377-387.

Pais-Vieira M, Lima D, and Galhardo V (2009) Sustained attention deficits in rats with chronic inflammatory pain. Neurosci Lett 463:98-102.

Pande AC, Pyke RE, Greiner M, Cooper SA, Benjamin R, and Pierce MW (1996) Analgesic efficacy of the kappa-receptor agonist, enadoline, in dental surgery pain. Clin Neuropharmacol 19:92-97.

Park HJ, Sandor K, McQueen J, Woller SA, Svensson CI, Corr M, and Yaksh TL (2016) The effect of gabapentin and ketorolac on allodynia and conditioned place preference in antibody-induced inflammation. Eur J Pain 20:917-925.

Park HJ, Stokes JA, Pirie E, Skahen J, Shtaerman Y, and Yaksh TL (2013) Persistent hyperalgesia in the cisplatin-treated mouse as defined by threshold measures, the conditioned place preference paradigm, and changes in dorsal root ganglia activated transcription factor 3: the effects of gabapentin, ketorolac, and etanercept. Anesth Analg 116:224-231.

Park JY and Wu LT (2017) Prevalence, reasons, perceived effects, and correlates of medical marijuana use: a review. Drug Alcohol Depend 177:1-13.

Pascual D, Goicoechea C, Suardíaz M, and Martín MI (2005) A cannabinoid agonist, WIN 55,212-2, reduces neuropathic nociception induced by paclitaxel in rats. Pain 118:23-34.

Patel JB and Migler B (1982) A sensitive and selective monkey conflict test. Pharmacol Biochem Behav 17:645-649.

Pavlov IP (1927) Conditioned Reflexes: An Investigation of the Physiological Activity of the Cerebral Cortex, Oxford University Press, Oxford, UK.

Pedersen LH and Blackburn-Munro G (2006) Pharmacological characterisation of place escape/avoidance behaviour in the rat chronic constriction injury model of neuropathic pain. Psychopharmacology (Berl) 185:208-217.

Pereira Do Carmo G, Stevenson GW, Carlezon WA, and Negus SS (2009) Effects of pain- and analgesia-related manipulations on intracranial self-stimulation in rats: further studies on pain-depressed behavior. Pain 144:170-177.

Pergolizzi JV Jr, LeQuang JA, Berger GK, and Raffa RB (2017) The basic pharmacology of opioids informs the opioid discourse about misuse and abuse: a review. Pain Ther 6:1-16.

Petraschka M, Li S, Gilbert TL, Westenbroek RE, Bruchas MR, Schreiber S, Lowe J, Low MJ, Pintar JE, and Chavkin C (2007) The absence of endogenous betaendorphin selectively blocks phosphorylation and desensitization of mu opioid receptors following partial sciatic nerve ligation. Neuroscience 146:1795-1807.

Pierce WD and Cheney CD (2017) Behavior Analysis and Learning, Routledge, New York.

Pollard GT and Howard JL (1990) Effects of drugs on punished behavior: pre-clinical test for anxiolytics. Pharmacol Ther 45:403-424.

Price DD, Finniss DG, and Benedetti F (2008) A comprehensive review of the placebo effect: recent advances and current thought. Annu Rev Psychol 59:565-590.

Price DD, Von der Gruen A, Miller J, Rafii A, and Price C (1985) A psychophysical analysis of morphine analgesia. Pain 22:261-269.

Raft D, Gregg J, Ghia J, and Harris L (1977) Effects of intravenous tetrahydrocannabinol on experimental and surgical pain. Psychological correlates of the analgesic response. Clin Pharmacol Ther 21:26-33.

Ramirez HE, Queeney TJ, Dunbar ML, Eichner MC, Del Castillo DI, Battles AH, and Neubert JK (2015) Assessment of an orofacial operant pain assay as a preclinical tool for evaluating analgesic efficacy in rodents. J Am Assoc Lab Anim Sci 54:426-432.

Ranjan R, Pandey S, and Shukla AK (2017) Biased opioid receptor ligands: gain without pain. Trends Endocrinol Metab 28:247-249.

Remeniuk B, Sukhtankar D, Okun A, Navratilova E, Xie JY, King T, and Porreca F (2015) Behavioral and neurochemical analysis of ongoing bone cancer pain in rats. Pain 156:1864-1873.

Ringkamp M, Raja SN, Campbell JN, and Meyer RA (2013) Peripheral mechanisms of cutaneous nociception, in Wall and Melzack's Textbook of Pain, 6th ed (McMahon SB, Koltzenburg M, Tracey I, and Turk D eds) pp 1-30, Elsevier, Philiadelphia. 
Ririe DG, Boada MD, MacGregor MK, Martin SJ, Strassburg TJ, Kim SA, Eisenach JC, and Martin T.J (2018) Incisional nociceptive input impairs attention-related behavior and is associated with reduced neuronal activity in the prefrontal cortex in rats. Anesthesiology 129:778-790.

Rohrs EL, Kloefkorn HE, Lakes EH, Jacobs BY, Neubert JK, Caudle RM, and Allen $\mathrm{KD}$ (2015) A novel operant-based behavioral assay of mechanical allodynia in the orofacial region of rats. $J$ Neurosci Methods 248:1-6.

Romero-Sandoval EA, Fincham JE, Kolano AL, Sharpe BN, and Alvarado-Vázquez PA (2018) Cannabis for chronic pain: challenges and considerations. Pharmacotherapy 38:651-662.

Rosenberg MB, Carroll FI, and Negus SS (2013) Effects of monoamine reuptake inhibitors in assays of acute pain-stimulated and pain-depressed behavior in rats. $J$ Pain 14:246-259.

Roughan JV, Coulter CA, Flecknell PA, Thomas HD, and Sufka KJ (2014) The conditioned place preference test for assessing welfare consequences and potential refinements in a mouse bladder cancer model. PLoS One 9:e103362.

Rowlett JK, Lelas S, Tornatzky W, and Licata SC (2006) Anti-conflict effects of benzodiazepines in rhesus monkeys: relationship with therapeutic doses in humans and role of GABAA receptors. Psychopharmacology (Berl) 184:201-211.

Rutten K, Robens A, Read SJ, and Christoph T (2014a) Pharmacological validation of a refined burrowing paradigm for prediction of analgesic efficacy in a rat model of sub-chronic knee joint inflammation. Eur J Pain 18:213-222.

Rutten K, Tzschentke TM, Koch T, Schiene K, and Christoph T (2014b) Pharmacogenomic study of the role of the nociceptin/orphanin $\mathrm{FQ}$ receptor and opioid receptors in diabetic hyperalgesia. Eur J Pharmacol 741:264-271.

Schuster CR and Johanson CE (1988) Relationship between the discriminative stimulus properties and subjective effects of drugs. Psychopharmacol Ser 4 161-175.

Seguin L, Le Marouille-Girardon S, and Millan MJ (1995) Antinociceptive profiles of non-peptidergic neurokinin1 and neurokinin2 receptor antagonists: a comparison to other classes of antinociceptive agent. Pain 61:325-343.

Semenchuk MR, Sherman S, and Davis B (2001) Double-blind, randomized trial of bupropion SR for the treatment of neuropathic pain. Neurology 57:1583-1588.

Sepinwall J, Grodsky FS, and Cook L (1978) Conflict behavior in the squirrel monkey: effects of chlordiazepoxide, diazepam and N-desmethyldiazepam. J Pharmacol Exp Ther 204:88-102.

Sheahan TD, Siuda ER, Bruchas MR, Shepherd AJ, Mohapatra DP, Gereau RW 4th, and Golden JP (2017) Inflammation and nerve injury minimally affect mouse voluntary behaviors proposed as indicators of pain. Neurobiol Pain 2:1-12.

Shepherd AJ, Cloud ME, Cao YQ, and Mohapatra DP (2018) Deficits in burrowing behaviors are associated with mouse models of neuropathic but not inflammatory pain or migraine. Front Behav Neurosci 12:124.

Shepherd AJ and Mohapatra DP (2018) Pharmacological validation of voluntary gait and mechanical sensitivity assays associated with inflammatory and neuropathic pain in mice. Neuropharmacology 130:18-29.

Sherrington CS (1906) The Integrative Action of the Nervous System, Scribner, New York.

Shippenberg TS and Herz A (1986) Differential effects of mu and kappa opioid systems on motivational processes. NIDA Res Monogr 75:563-566.

Shippenberg TS, Stein C, Huber A, Millan MJ, and Herz A (1988) Motivational effects of opioids in an animal model of prolonged inflammatory pain: alteration in the effects of kappa- but not of mu-receptor agonists. Pain 35:179-186.

Siegel S (1978) Morphine tolerance: is there evidence for a conditioning model? Science 200:344-345.

Simmons RM, Forster B, Guo W, and Knopp KL (2014) A method to enhance the magnitude of tactile hypersensitivity following spinal nerve ligation in rats. $J$ Neurosci Methods 233:50-53.

Skinner BF (1957) Verbal Behavior, Copley Publishing Group, Acton, MA.

Skinner BF (1974) About Behaviorism, Vintage Books, New York.

Smith FL, Fujimori K, Lowe J, and Welch SP (1998) Characterization of delta9tetrahydrocannabinol and anandamide antinociception in nonarthritic and arthritic rats. Pharmacol Biochem Behav 60:183-191.

Sofia RD, Vassar HB, and Knobloch LC (1975) Comparative analgesic activity of various naturally occurring cannabinoids in mice and rats. Psychopharmacology (Berl) 40:285-295.

Sotocinal SG, Sorge RE, Zaloum A, Tuttle AH, Martin LJ, Wieskopf JS, Mapplebeck JC, Wei P, Zhan S, Zhang S, et al. (2011) The Rat Grimace Scale: a partially automated method for quantifying pain in the laboratory rat via facial expressions. Mol Pain 7:55.

Sounvoravong S, Takahashi M, Nakashima MN, and Nakashima K (2004) Disability of development of tolerance to morphine and U-50,488H, a selective kappa-opioid receptor agonist, in neuropathic pain model mice. J Pharmacol Sci 94:305-312.

St. Sauver JL, Warner DO, Yawn BP, Jacobson DJ, McGree ME, Pankratz JJ, Melton LJ 3rd, Roger VL, Ebbert JO, and Rocca WA (2013) Why patients visit their doctors: assessing the most prevalent conditions in a defined American population. Mayo Clin Proc 88:56-67.

Stein C, Millan MJ, and Herz A (1988) Unilateral inflammation of the hindpaw in rats as a model of prolonged noxious stimulation: alterations in behavior and nociceptive thresholds. Pharmacol Biochem Behav 31:445-451.

Stevenson GW, Bilsky EJ, and Negus SS (2006) Targeting pain-suppressed behaviors in preclinical assays of pain and analgesia: effects of morphine on acetic acidsuppressed feeding in C57BL/6J mice. J Pain 7:408-416.

Stevenson GW, Cormier J, Mercer H, Adams C, Dunbar C, Negus SS, and Bilsky EJ (2009) Targeting pain-depressed behaviors in preclinical assays of pain and analgesia: drug effects on acetic acid-depressed locomotor activity in ICR mice. Life Sci 85:309-315.

Stevenson GW, Mercer H, Cormier J, Dunbar C, Benoit L, Adams C, Jezierski J, Luginbuhl A, and Bilsky EJ (2011) Monosodium iodoacetate-induced osteoarthritis produces pain-depressed wheel running in rats: implications for preclinical behavioral assessment of chronic pain. Pharmacol Biochem Behav 98:35-42.
Stockings E, Campbell G, Hall WD, Nielsen S, Zagic D, Rahman R, Murnion B, Farrell M, Weier M, and Degenhardt L (2018) Cannabis and cannabinoids for the treatment of people with chronic noncancer pain conditions: a systematic review and meta-analysis of controlled and observational studies. Pain 159:1932-1954.

Sufka KJ (1994) Conditioned place preference paradigm: a novel approach for analgesic drug assessment against chronic pain. Pain 58:355-366.

Suzuki T, Kishimoto Y, and Misawa M (1996) Formalin- and carrageenan-induced inflammation attenuates place preferences produced by morphine, methamphetamine and cocaine. Life Sci 59:1667-1674.

Takada K, Barrett JE, Allen MS, Cook JM, and Katz JL (1992) Punishment of schedule-controlled behavior with beta-carboline injections: antagonism and comparisons with other compounds. J Pharmacol Exp Ther 261: 138-145.

Tanimoto S, Nakagawa T, Yamauchi Y, Minami M, and Satoh M (2003) Differential contributions of the basolateral and central nuclei of the amygdala in the negative affective component of chemical somatic and visceral pains in rats. Eur J Neurosci 18:2343-2350.

Terrando N, Brzezinski M, Degos V, Eriksson LI, Kramer JH, Leung JM, Miller BL, Seeley WW, Vacas S, Weiner MW, et al. (2011) Perioperative cognitive decline in the aging population. Mayo Clin Proc 86:885-893.

Thomas DA, Oliveras JL, Maixner W, and Dubner R (1992) Systemic morphine administration attenuates the perceived intensity of noxious heat in the monkey. Pain 49:129-135

Toma W, Kyte SL, Bagdas D, Alkhlaif Y, Alsharari SD, Lichtman AH, Chen ZJ, Del Fabbro E, Bigbee JW, Gewirtz DA, et al. (2017) Effects of paclitaxel on the development of neuropathy and affective behaviors in the mouse. Neuropharmacology 117:305-315.

Tseng AH and Craft RM (2001) Sex differences in antinociceptive and motoric effects of cannabinoids. Eur J Pharmacol 430:41-47.

Tzschentke TM (2007) Measuring reward with the conditioned place preference (CPP) paradigm: update of the last decade. Addict Biol 12:227-462.

Urban R, Scherrer G, Goulding EH, Tecott LH, and Basbaum AI (2011) Behavioral indices of ongoing pain are largely unchanged in male mice with tissue or nerve injury-induced mechanical hypersensitivity. Pain 152:990-1000.

Vadivelu N, Kai AM, Kodumudi G, Sramcik J, and Kaye AD (2018) Medical marijuana: current concepts, pharmacological actions of cannabinoid receptor mediated activation, and societal implications. Curr Pain Headache Rep 22:3.

Vadivelu N, Mitra S, and Hines RL (2011) Peripheral opioid receptor agonists for analgesia: a comprehensive review. J Opioid Manag 7:55-68.

van der Kam EL, Vry JD, Schiene K, and Tzschentke TM (2008) Differential effects of morphine on the affective and the sensory component of carrageenan-induced nociception in the rat. Pain 136:373-379.

Vanderah TW, Largent-Milnes T, Lai J, Porreca F, Houghten RA, Menzaghi F, Wisniewski K, Stalewski J, Sueiras-Diaz J, Galyean R, et al. (2008) Novel D-amino acid tetrapeptides produce potent antinociception by selectively acting at peripheral kappa-opioid receptors. Eur J Pharmacol 583:62-72.

Vanderah TW, Ossipov MH, Lai J, Malan TP Jr, and Porreca F (2001) Mechanisms of opioid-induced pain and antinociceptive tolerance: descending facilitation and spinal dynorphin. Pain 92:5-9.

Vierck CJ, Acosta-Rua A, Nelligan R, Tester N, and Mauderli A (2002) Low dose systemic morphine attenuates operant escape but facilitates innate reflex responses to thermal stimulation. J Pain 3:309-319.

Vierck CJ, Hansson PT, and Yezierski RP (2008) Clinical and pre-clinical pain assessment: are we measuring the same thing? Pain 135:7-10.

Volkow ND and Collins FS (2017) The role of science in addressing the opioid crisis. $N$ Engl J Med 377:391-394.

von Baeyer CL, Chambers CT, and Eakins DM (2011) Development of a 10-item short form of the parents' postoperative pain measure: the PPPM-SF. J Pain 12:401-406. Walker G (2018) The opioid crisis: a 21st century pain. Drugs Today (Barc) 54:283-286

Wallace M, Schulteis G, Atkinson JH, Wolfson T, Lazzaretto D, Bentley H, Gouaux B, and Abramson I (2007) Dose-dependent effects of smoked cannabis on capsaicininduced pain and hyperalgesia in healthy volunteers. Anesthesiology 107:785-796.

Wang HC, Chai SC, Wu YS, and Wang CC (2007) Does the medial thalamus play a role in the negative affective component of visceral pain in rats? Neurosci Lett 420: $80-84$.

Wang HC, Wang YC, Huang AC, Chai SC, Wu YS, and Wang CC (2009) Roles of corticosterone in formalin-induced conditioned place aversion in rats. Neurosci Let 464:122-126.

Wang YJ, Zuo ZX, Wu C, Liu L, Feng ZH, and Li XY (2017) Cingulate alpha-2a adrenoceptors mediate the effects of clonidine on spontaneous pain induced by peripheral nerve injury. Front Mol Neurosci 10:289.

Watanabe M and Narita M (2018) Brain reward circuit and pain. Adv Exp Med Biol 1099:201-210.

Westbrook RF, Greeley JD, Nabke CP, and Swinbourne AL (1991) Aversive conditioning in the rat: effects of a benzodiazepine and of an opioid agonist and antagonist on conditioned hypoalgesia and fear. J Exp Psychol Anim Behav Process 17:219-230.

Whiteside GT, Pomonis JD, and Kennedy JD (2016) Preclinical pharmacological approaches in drug discovery for chronic pain. Adv Pharmacol 75:303-323.

Whitlock EL and Pardo MC (2017) Choice of anesthetic technique, in Basics of Anesthesia, 7th ed (Pardo MC and Miller RD eds) pp 213-220, Elsevier, Philadelphia.

Wiffen PJ, Derry S, Bell RF, Rice AS, Tölle TR, Phillips T, and Moore RA (2017) Gabapentin for chronic neuropathic pain in adults. Cochrane Database Syst Rev 6:CD007938

Wilkerson JL, Curry ZA, Kinlow PD, Mason BL, Hsu KL, van der Stelt M, Cravatt BF, and Lichtman AH (2018) Evaluation of different drug classes on transient sciatic nerve injury-depressed marble burying in mice. Pain 159: 1155-1165. 
Wodarski R, Delaney A, Ultenius C, Morland R, Andrews N, Baastrup C, Bryden LA, Caspani O, Christoph T, Gardiner NJ, et al. (2016) Crosscentre replication of suppressed burrowing behaviour as an ethologically relevant pain outcome measure in the rat: a prospective multicentre study. Pain 157:2350-2365.

Woller SA, Moreno GL, Hart N, Wellman PJ, Grau JW, and Hook MA (2012) Analgesia or addiction?: implications for morphine use after spinal cord injury. J Neurotrauma 29:1650-1662.

Wood PB (2008) Role of central dopamine in pain and analgesia. Expert Rev Neurother 8:781-797.

Woolf CJ and Ma Q (2007) Nociceptors--noxious stimulus detectors. Neuron 55: 353-364.

Xie JY, Qu C, Patwardhan A, Ossipov MH, Navratilova E, Becerra L, Borsook D, and Porreca F (2014) Activation of mesocorticolimbic reward circuits for assessment of relief of ongoing pain: a potential biomarker of efficacy. Pain 155:1659-1666.

Xu M, Petraschka M, McLaughlin JP, Westenbroek RE, Caron MG, Lefkowitz RJ, Czyzyk TA, Pintar JE, Terman GW, and Chavkin C (2004) Neuropathic pain activates the endogenous kappa opioid system in mouse spinal cord and induces opioid receptor tolerance. J Neurosci 24:4576-4584.

Yezierski RP and Hansson P (2018) Inflammatory and neuropathic pain from bench to bedside: what went wrong? J Pain 19:571-588.

Yu D (2011) Translational research: current status, challenges and future strategies. Am J Transl Res 3:422-433.

Zhang X, Xin X, Dong Y, Zhang Y, Yu B, Mao J, and Xie Z (2013) Surgical incisioninduced nociception causes cognitive impairment and reduction in synaptic NMDA receptor $2 \mathrm{~B}$ in mice. $J$ Neurosci 33:17737-17748.

Zhang Y, Meng X, Li A, Xin J, Berman BM, Lao L, Tan M, Ren K, and Zhang RX (2012) Electroacupuncture alleviates affective pain in an inflammatory pain rat model. Eur J Pain 16:170-181.

Zhang Z, Tao W, Hou YY, Wang W, Kenny PJ, and Pan ZZ (2014) MeCP2 repression of G9a in regulation of pain and morphine reward. J Neurosci 34:9076-9087.

Zuo Z (2005) The role of opioid receptor internalization and beta-arrestins in the development of opioid tolerance. Anesth Analg 101:728-734. 